\title{
STABILITY AND SENSITIVITY ANALYSIS FOR OPTIMAL CONTROL PROBLEMS WITH A FIRST-ORDER STATE CONSTRAINT AND APPLICATION TO CONTINUATION METHODS
}

\author{
Joseph Frédéric Bonnans ${ }^{1}$ And Audrey Hermant ${ }^{1}$
}

\begin{abstract}
The paper deals with an optimal control problem with a scalar first-order state constraint and a scalar control. In presence of (nonessential) touch points, the arc structure of the trajectory is not stable. Under some reasonable assumptions, we show that boundary arcs are structurally stable, and that touch point can either remain so, vanish or be transformed into a single boundary arc. Assuming a weak second-order optimality condition (equivalent to uniform quadratic growth), stability and sensitivity results are given. The main tools are the study of a quadratic tangent problem and the notion of strong regularity. Those results enable us to design a new continuation algorithm, presented at the end of the paper, that handles automatically changes in the structure of the trajectory.
\end{abstract}

Mathematics Subject Classification. 49K40, 49N60, 34B15.

Received July 24, 2006. Revised May 2, 2007.

Published online February 7, 2008.

\section{INTRODUCTION}

This paper deals with an optimal control problem (of an ordinary differential equation) with a scalar firstorder state constraint and a scalar control, with a free final state and no control constraints. It is well-known that for first-order state constraints, when the strengthened Legendre-Clebsch condition holds and the state constraint is regular, touch points (locally unique times where the constraint is active) are nonessential (the associated jump of the multiplier is null) (see e.g. $[17,19]$ ). Situations where touch points are present may be encountered, for instance, when solving the optimal control problem by indirect approaches using an homotopy method in order to guess the arc structure of the trajectory, see e.g. the example in [3]. Therefore it is of interest to study sensitivity of solutions around touch points, when the constraint becomes active. Under a small perturbation, several events may occur. Among them, the constraint may locally become inactive, the touch point may remain a touch point, or it may give rise to a boundary arc. Our main result is that, under natural hypotheses, these are the only three possibilities, and that the boundary arcs have a length of the order of the perturbation, and satisfy a "strict complementarity" hypothesis. In addition, we show how to compute a first-order expansion of the solution. The analysis uses in a critical way a certain tangent quadratic problem,

\footnotetext{
Keywords and phrases. Optimal control, first-order state constraint, strong regularity, sensitivity analysis, touch point, homotopy method.

${ }^{1}$ INRIA Saclay and Centre de Mathématiques Appliquées, École Polytechnique, 91128 Palaiseau, France;

Frederic.Bonnans@inria.fr; hermant@cmap.polytechnique.fr
} 
and at the same time is in the spirit of the shooting approach, in the sense that touch points are converted into boundary arcs of zero length, and we compute the first-order expansion of all entry and exit points. Fréchet directional derivatives are obtained as the solution of an inequality-constrained linear quadratic problem. The proof applies the notion of "strong regularity" in the sense of Robinson [31] to a system that happens to be equivalent to the optimality conditions of the tangent quadratic problem. Our formulation of the corresponding shooting formulation (of which all entry and exit times are variables, in addition to the initial costate and jumps of the alternative multiplier at entry times) allows exit times to be lower than entry times; however, we check that the solution of the shooting formulation is such that entry times are lower than or equal to corresponding exit times.

Optimal control problems with first-order state constraints were first studied in the book by Pontryagin et al. [28]. Numerous results have been obtained since for stability and sensitivity analysis of those problems. Two different approaches have been used. The first one is the use of implicit function theorems in infinite dimensional spaces (see $[16,20,27,32]$ ), and the second one is to reduce the problem to a finite-dimensional one (a two- or multi points boundary value problem) using the so-called shooting formulation (see $[26,33]$ ). With first-order state constraints, $L^{2}$-stability of solutions was first obtained by Malanowski [21], under strong second-order sufficient conditions, using an infinite-dimensional implicit function theorem based on two-norms approach, and later by Dontchev and Hager [12], using an implicit function theorem in metric spaces. In Malanowski [21], directional differentiability of solutions in $L^{2}$ was established, using the results on differentiability of projection onto a closed convex cone in Hilbert spaces [16]. The second-order sufficient condition used in the analysis was weakened by Malanowski [22]. All those results require no assumptions on the structure of the trajectory. In order to obtain $L^{\infty}$-stability of solutions, Dontchev and Hager [12] needed an additional assumption on the structure of the contact set ("contact separation"). Using a finite dimensional approach, Malanowski and Maurer obtained in [23] differentiability of solutions in $L^{\infty}$ by application of the implicit function theorem to the shooting mapping, under stronger assumptions (finitely many nontangential junction points, and strict complementarity) needed to ensure the stability of the structure of solutions.

The approach presented in this paper is different from the ones in $[12,21,22]$ where the stability and sensitivity analysis was done in infinite dimensional spaces without any assumptions on the structure of the trajectory. On the contrary, our aim is to describe changes in the structure of the trajectory, both qualitatively and quantitatively. Thus the first step is to consider nonessential touch points. Indeed, as mentioned before, changes in the structure are likely to occur when performing continuation methods, therefore the more information we have on the continuity and/or differentiability of the homotopy path, the easier will be the latter to follow. Our stability and sensitivity results generalize those of [23] to the case when (nonessential) touch points are present. However, in that case strict complementarity does not hold anymore, so we cannot apply the classical implicit function theorem as done in [23]. This paper is related to our previous work: the study of no-gap second-order optimality conditions in [7], and the shooting formulation, allowing nonessential touch points for state constraints of order greater than one, and for which we also use the notion of strong regularity [6]. In both papers we assume also the state constraint and the control to be scalar-valued. Some of these results are extended to the case of vector-valued state constraints and control in [5]. We follow here the analysis in [6] where sensitivity results with nonessential touch points for state constraints of order greater than one were obtained. The contributions of this paper are the following:

- A stability result of the structure of stationary points (and not only the stability of the structure of locally optimal solutions) is proved. That is, if the nominal trajectory satisfies several assumptions, among which uniform strict complementarity on boundary arcs, then any stationary point in the neighborhood has a "neighboring structure", in a sense made precise in Section 2.

- In the stability and sensitivity analysis we cover the case of the possible transformation of touch points into boundary arcs. This possibility was excluded from the analysis in [6] and in [23], and leads to technical complications. In particular we show that for first-order state constraints, the shooting algorithm remain well-posed when touch points are converted in boundary arcs, which is false for control constraints (see Rem. 6.3). 
- At the end of the paper, we present an application of those results to a preliminary homotopy algorithm whose novelty is to handle changes in the structure (appearance/disappearance of a boundary arc) automatically. Numerical application on a simple academic problem is presented.

The paper is organized at follows. The framework is presented in Section 1. In Section 2, the stability results of the structure of stationary points are given. In Section 3, the main result is stated. In Section 4, the problem is reduced to a generalized finite-dimensional equation, with a complementarity constraint. Robinson's strong regularity theory is applied to the latter in Section 5, where the main result is proved. Section 6 deals with directional differentiability of solutions. In Section 7, a basic illustrative example is presented. The homotopy method is described in Section 8. Section 9 contains the proofs of the results of Section 2.

\section{Preliminaries}

Let $\mathcal{U}:=L^{\infty}(0, T)\left(\right.$ resp. $\left.\mathcal{Y}:=W^{1, \infty}\left(0, T ; \mathbb{R}^{n}\right)\right)$ denote the control (resp. state) space. Let $M$ be a Banach space (the space of perturbations parameter) and, for $\mu \in M$, the cost function $\ell^{\mu}: \mathbb{R} \times \mathbb{R}^{n} \rightarrow \mathbb{R}$, final cost function $\phi^{\mu}: \mathbb{R}^{n} \rightarrow \mathbb{R}$, dynamics $f^{\mu}: \mathbb{R} \times \mathbb{R}^{n} \rightarrow \mathbb{R}^{n}$, state constraint $g^{\mu}: \mathbb{R}^{n} \rightarrow \mathbb{R}$, initial condition $y_{0}^{\mu} \in \mathbb{R}^{n}$, and (fixed) final time $T>0$. We consider the following optimal control problem:

$$
\begin{aligned}
\left(\mathcal{P}^{\mu}\right) & \min _{(u, y) \in \mathcal{U} \times \mathcal{Y}} \int_{0}^{T} \ell^{\mu}(u(t), y(t)) \mathrm{d} t+\phi^{\mu}(y(T)) \\
\text { subject to } & \dot{y}(t)=f^{\mu}(u(t), y(t)) \text { for a.a. } t \in[0, T], \quad y(0)=y_{0}^{\mu}, \\
& g^{\mu}(y(t)) \leq 0, \text { for all } t \in[0, T] .
\end{aligned}
$$

This notation allow us to deal with non autonomous problems (i.e. when the data $\ell^{\mu}, f^{\mu}, g^{\mu}$ depend on time $t$ ) as well, by assuming w.l.o.g. that the last component of the state variable $y_{n}$ satisfies in (1.2)

$$
\left.\dot{y}_{n}(t)=1 \quad \text { on }[0, T], \quad y_{n}(0)=0 \quad \text { (i.e. } y_{n}(t)=t\right) \text {. }
$$

We shall assume in all the paper that $\left(\mathcal{P}^{\mu}\right)$ is written such that (1.4) holds. In this way our analysis will include non autonomous perturbations, even when the starting problem is autonomous. This assumption is only used in Theorem 3.3 to obtain the implication (i) $\Rightarrow$ (ii).

We study perturbations of problem $\left(\mathcal{P}^{\mu}\right)$ around a given value of parameter $\mu_{0} \in M$, and we often omit the superscript $\mu$ when we refer to the problem and data associated with $\mu_{0}$, i.e. $(\mathcal{P}):=\left(\mathcal{P}^{\mu_{0}}\right)$ and $\left(\ell, \phi, f, g, y_{0}\right):=$ $\left(\ell^{\mu_{0}}, \phi^{\mu_{0}}, f^{\mu_{0}}, g^{\mu_{0}}, y_{0}^{\mu_{0}}\right)$.

We assume throughout the paper that the assumptions below hold:

(A0) the mappings $\ell, \phi, f$ and $g$ are of class $C^{2}$, with locally Lipschitz continuous second-order derivatives, and the dynamics $f$ is Lipschitz continuous;

(A1) the initial condition satisfies $g\left(y_{0}\right)<0$.

These assumptions will not be repeated in the various results of the paper.

A parameterization $\left(\ell^{\mu}, \phi^{\mu}, f^{\mu}, g^{\mu}, y_{0}^{\mu}\right)$, identified with problem $\left(\mathcal{P}^{\mu}\right)$, is a stable extension of $(\mathcal{P})$, if there exists an open neighborhood $M_{0}$ of $\mu_{0}$, such that (i) there exist $C^{2}$ mappings $\hat{\ell}: \mathbb{R} \times \mathbb{R}^{n} \times M_{0} \rightarrow \mathbb{R} ; \hat{\phi}:$ $\mathbb{R}^{n} \times M_{0} \rightarrow \mathbb{R} ; \hat{f}: \mathbb{R} \times \mathbb{R}^{n} \times M_{0} \rightarrow \mathbb{R}^{n} ; \hat{g}: \mathbb{R}^{n} \times M_{0} \rightarrow \mathbb{R}$ and $\hat{y}_{0}: M_{0} \rightarrow \mathbb{R}^{n}$, such that $\ell^{\mu}(u, y)=\hat{\ell}(u, y, \mu)$ for all $(u, y) \in \mathbb{R} \times \mathbb{R}^{n}$ and all $\mu \in M_{0}$ (and similarly for $\phi^{\mu}, f^{\mu}, g^{\mu}$, and $y_{0}^{\mu}$ ); (ii) the mappings $\ell^{\mu}, f^{\mu}, \phi^{\mu}, g^{\mu}$ have Lipschitz continuous second-order derivatives and $f^{\mu}$ is Lipschitz continuous, uniformly over $\mu \in M_{0}$.

In this paper, we always consider stable extensions $\left(\mathcal{P}^{\mu}\right)$, that satisfy (1.4) as said before.

\section{Definitions and notations}

The space of row vectors is denoted by $\mathbb{R}^{n *}$, and the adjoint and transposition operator in $\mathbb{R}^{n}$ are denoted by a $\operatorname{star}^{*}$. Fréchet derivatives of $f$, $\ell$, etc. w.r.t. arguments $u \in \mathbb{R}, y \in \mathbb{R}^{n}$, are denoted by a subscript, for instance $f_{u}(u, y)=D_{u} f(u, y)$. The space $L^{r}(0, T), r \in[1, \infty]$, is the Lebesgue space of measurable functions 
such that $\|u\|_{r}:=\left(\int_{0}^{T}|u(t)|^{r}\right)^{1 / r}<\infty$ for $1 \leq r<\infty$ and $\|u\|_{\infty}:=\operatorname{supess}_{t \in[0, T]}|u(t)|<\infty$, and $W^{1, r}(0, T)$ is the Sobolev space of functions in $L^{r}(0, T)$ with a weak derivative in $L^{r}(0, T)$. The space of continuous functions and its dual space, the space of bounded Borel measures, are denoted respectively by $C^{0}[0, T]$ and $\mathcal{M}[0, T]$. The cone of nonnegative measures is denoted by $\mathcal{M}_{+}[0, T]$, and $B V\left([0, T] ; \mathbb{R}^{n}\right)$ denotes the space of vector-valued functions of bounded variation over $[0, T]$. The elements of $\mathcal{M}[0, T]$ are identified with the derivative of functions of bounded variation vanishing at $T$. We denote by $\varphi\left(t^{-}\right)$and $\varphi\left(t^{+}\right)$the respectively left- and right limits of a function of bounded variation $\varphi$ at a time $t \in[0, T]$. Jumps are denoted by $[\varphi(t)]:=\varphi\left(t^{+}\right)-\varphi\left(t^{-}\right)$.

Given $\mu \in M_{0}$, a trajectory of $\left(\mathcal{P}^{\mu}\right)$ is an element $(u, y) \in \mathcal{U} \times \mathcal{Y}$ satisfying the state equation (1.2). A feasible trajectory is one satisfying the state constraint (1.3). The first-order time derivative of the state constraint is the function defined by $\left(g^{\mu}\right)^{(1)}: \mathbb{R} \times \mathbb{R}^{n} \rightarrow \mathbb{R},(u, y) \mapsto g_{y}^{\mu}(y) f^{\mu}(u, y)$. In this paper, we consider state constraints of first order, that is, the function $\left(g^{\mu}\right)^{(1)}(u, y)$ depends explicitly on the control variable $u$ in the neighborhood of the contact set of the constraint, see assumption (A3). It will be convenient to introduce the second-order time derivative of the state constraint by:

$$
\left(g^{\mu}\right)^{(2)}: \mathbb{R} \times \mathbb{R} \times \mathbb{R}^{n} \rightarrow \mathbb{R}, \quad(v, u, y) \mapsto\left(g^{\mu}\right)_{u}^{(1)}(u, y) v+\left(g^{\mu}\right)_{y}^{(1)}(u, y) f^{\mu}(u, y) .
$$

Wherever $u$ is differentiable, we have that

$$
\frac{\mathrm{d}^{2}}{\mathrm{~d} t^{2}} g^{\mu}(y(t))=\left(g^{\mu}\right)^{(2)}(\dot{u}(t), u(t), y(t))
$$

The classical (resp. augmented) Hamiltonian functions $H^{\mu}: \mathbb{R} \times \mathbb{R}^{n} \times \mathbb{R}^{n *} \rightarrow \mathbb{R}\left(\right.$ resp. $\tilde{H}^{\mu}: \mathbb{R} \times \mathbb{R}^{n} \times \mathbb{R}^{n *} \times \mathbb{R} \rightarrow$ $\mathbb{R})$ are defined by:

$$
\begin{aligned}
H^{\mu}(u, y, p) & :=\ell^{\mu}(u, y)+p f^{\mu}(u, y) \\
\tilde{H}^{\mu}\left(u, y, p^{1}, \eta^{1}\right) & :=H^{\mu}\left(u, y, p^{1}\right)+\eta^{1}\left(g^{\mu}\right)^{(1)}(u, y) .
\end{aligned}
$$

For $(u, y)$ a feasible trajectory of $\left(\mathcal{P}^{\mu}\right)$, define the contact set by:

$$
I\left(g^{\mu}(y)\right):=\left\{t \in[0, T] ; g^{\mu}(y(t))=0\right\} .
$$

We say that the constraint is active at time $t$, if $t \in I\left(g^{\mu}(y)\right)$; otherwise it is said inactive at time $t$. A boundary arc (resp. interior arc) is a maximal interval of positive measure $\mathcal{I}$ such that $g^{\mu}(y(t))=0$ (resp. $\left.g^{\mu}(y(t))<0\right)$, for all $t \in \mathcal{I}$. Left and right endpoints of a boundary $\operatorname{arc}\left[\tau_{\mathrm{en}}, \tau_{\mathrm{ex}}\right]$ are called entry and exit point, respectively. A touch point $\tau_{\text {to }}$ is an isolated contact point, satisfying $g^{\mu}\left(y\left(\tau_{\text {to }}\right)\right)=0$ and $g^{\mu}(y(t))<0$, for $t \neq \tau_{\text {to }}$ in the neighborhood of $\tau_{\text {to }}$. The endpoints of interior arcs belonging to $(0, T)$ are called junction points (or times).

If the set of junction points of a trajectory is finite, then it is of the form

$$
\mathcal{T}=: \mathcal{T}_{\text {en }} \cup \mathcal{T}_{\text {ex }} \cup \mathcal{T}_{\text {to }},
$$

with $\mathcal{T}_{\text {en }}, \mathcal{T}_{\text {ex }}$ and $\mathcal{T}_{\text {to }}$ the disjoint (and possibly empty) subsets of respectively regular entry, exit and touch points. We denote by $\mathcal{I}_{b}$ the union of boundary arcs, i.e. $\mathcal{I}_{b}:=\cup_{i=1}^{N_{b}}\left[\tau_{i}^{\mathrm{en}}, \tau_{i}^{\mathrm{ex}}\right]$ for $\mathcal{T}_{\mathrm{en}}:=\left\{\tau_{1}^{\mathrm{en}}<\ldots<\tau_{N_{b}}^{\mathrm{en}}\right\}$ and similar definition of $\mathcal{T}_{\text {ex }}$, and we have $I\left(g^{\mu}(y)\right)=\mathcal{T}_{\text {to }} \cup \mathcal{I}_{b}$. The arc structure (or simply structure) of a trajectory is the (finite) number of boundary arcs and touch points, and the order in which they occur.

Given a finite subset $\mathcal{S}$ of $(0, T)$, we denote by $P C_{\mathcal{S}}^{k}[0, T]$ the set of functions over $[0, T]$ that are of class $C^{k}$ outside $\mathcal{S}$, and have, as well as their first $k$ derivatives, a left and right limit over $\mathcal{S}$ and a left (resp. right) limit at $T$ (resp. 0). The subset of functions in $P C_{\mathcal{S}}^{k}[0, T]$ having continuous derivatives on $[0, T]$ until order $r$, $0 \leq r \leq k$, is denoted by $P C_{\mathcal{S}}^{k, r}[0, T]:=P C_{\mathcal{S}}^{k}[0, T] \cap C^{r}[0, T]$. We also use the notation $\nu_{\mathcal{S}}:=\left(\nu_{\tau}\right)_{\tau \in \mathcal{S}} \in \mathbb{R}^{\text {Card } \mathcal{S}}$.

Given $(\mu, u) \in M_{0} \times \mathcal{U}$, we denote by $y_{u}^{\mu}$ the (unique) state solution in $\mathcal{Y}$ of:

$$
\dot{y}_{u}^{\mu}(t)=f^{\mu}\left(u(t), y_{u}^{\mu}(t)\right) \quad \text { a.e. on }[0, T], \quad y_{u}^{\mu}(0)=y_{0}^{\mu} .
$$


By definition of a stable extension, the mapping $\mathcal{U} \times M_{0} \rightarrow \mathcal{Y},(u, \mu) \mapsto y_{u}^{\mu}$ is $C^{2}$. A useful equivalent abstract formulation of $\left(\mathcal{P}^{\mu}\right)$ is

$$
\min _{u \in \mathcal{U}} J^{\mu}(u), \quad G^{\mu}(u) \in K,
$$

with the cost function $J^{\mu}: \mathcal{U} \rightarrow \mathbb{R}, u \mapsto \int_{0}^{T} \ell^{\mu}\left(u(t), y_{u}^{\mu}(t)\right) \mathrm{d} t+\phi^{\mu}\left(y_{u}^{\mu}(T)\right), K:=C_{-}^{0}[0, T]$ the cone of continuous functions taking nonpositive values, and $G^{\mu}$ the mapping $\mathcal{U} \rightarrow C^{0}[0, T], u \mapsto g^{\mu}\left(y_{u}^{\mu}\right)$. We write $J$ and $G$ for $J^{\mu_{0}}$ and $G^{\mu_{0}}$, respectively.

\section{Optimality conditions}

Let us first recall the definition of Pontryagin extremals.

Definition 1.1. A trajectory $(u, y)$ is a Pontryagin extremal of $\left(\mathcal{P}^{\mu}\right)$, if there exist $\alpha \in \mathbb{R}_{+}, \mathrm{d} \eta \in \mathcal{M}[0, T]$ and $p \in B V\left([0, T] ; \mathbb{R}^{n *}\right),(\mathrm{d} \eta, p, \alpha) \neq 0$, such that:

$$
\begin{aligned}
\dot{y}(t) & =f^{\mu}(u(t), y(t)) \quad \text { a.e. on }[0, T], \quad y(0)=y_{0}^{\mu} \\
\mathrm{d} p(t) & =\left\{\alpha \ell_{y}^{\mu}(u(t), y(t))+p(t) f_{y}^{\mu}(u(t), y(t))\right\} \mathrm{d} t+g_{y}^{\mu}(y(t)) \mathrm{d} \eta(t) \quad \text { on }[0, T] \\
p\left(T^{+}\right) & =\alpha \phi_{y}^{\mu}(y(T)) \\
u(t) & \in \operatorname{argmin}_{\hat{u} \in \mathbb{R}}\left\{\alpha \ell^{\mu}(\hat{u}, y(t))+p(t) f^{\mu}(\hat{u}, y(t))\right\} \quad \text { a.e. on }[0, T] \\
0 & \geq g^{\mu}(y(t)), \quad \mathrm{d} \eta \geq 0, \quad \int_{0}^{T} g^{\mu}(y(t)) \mathrm{d} \eta(t)=0 .
\end{aligned}
$$

When $\alpha>0$, dividing $p$ and $\eta$ by $\alpha$, we can take $\alpha=1$ in the above equations, and in that case we say that $(u, y)$ is a regular Pontryagin extremal.

It is well known that optimal solutions of $\left(\mathcal{P}^{\mu}\right)$ are Pontryagin extremals. A sufficient condition to ensure that $\alpha=1$, i.e. that an optimal solution $(u, y)$ of $\left(\mathcal{P}^{\mu}\right)$ is a regular Pontryagin extremal, is that Robinson's constraint qualification $[29,30]$ below is satisfied (recall $(1.11)$ ):

$$
\exists \gamma>0, \quad \gamma B_{C^{0}[0, T]} \subset G^{\mu}(u)+D G^{\mu}(u) \mathcal{U}-K,
$$

with $B_{C^{0}[0, T]}$ the unit (open) ball of the space of continuous functions.

A trajectory $(u, y)$ is a stationary point of $\left(\mathcal{P}^{\mu}\right)$, if there exist $\mathrm{d} \eta \in \mathcal{M}[0, T]$ and $p \in B V\left([0, T] ; \mathbb{R}^{n *}\right)$ such that (1.12)-(1.14) and (1.16) hold (with $\alpha=1$ ), as well as

$$
0=\ell_{u}^{\mu}(u(t), y(t))+p(t) f_{u}^{\mu}(u(t), y(t)) \quad \text { for a.a. } t \in[0, T] .
$$

The above condition is in general weaker than (1.15). However, when the Hamiltonian $H^{\mu}$ is convex w.r.t. the control variable along the trajectory (and in particular when assumption (1.22) below holds), then the definitions of regular Pontryagin extremals and stationary points are equivalent.

We say that $(u, y)$ is a local solution (weak minimum) of $\left(\mathcal{P}^{\mu}\right)$, if it minimizes (1.1) over the set of feasible trajectories $(\tilde{u}, \tilde{y})$ satisfying $\|\tilde{u}-u\|_{\infty} \leq \delta$ for some $\delta>0$. Local solutions of $\left(\mathcal{P}^{\mu}\right)$ satisfying (1.17) are stationary points.

Note that the complementarity conditions (1.16) can be equivalently rewritten as:

$$
g^{\mu}(y) \in K, \quad \mathrm{~d} \eta \in \mathcal{M}_{+}[0, T], \quad \operatorname{supp}(\mathrm{d} \eta) \subset I\left(g^{\mu}(y)\right),
$$

where $\operatorname{supp}(\mathrm{d} \eta)$ denotes the support of the measure $\mathrm{d} \eta$. Another condition equivalent to (1.16) is $\mathrm{d} \eta \in$ $N_{K}\left(G^{\mu}(u)\right.$ ), where $N_{K}\left(G^{\mu}(u)\right)$ denotes the normal cone (in the sense of convex analysis) to $K$ at point $G^{\mu}(u)$. 


\section{Assumptions}

We assume that problem $(\mathcal{P})$ has a local solution, denoted in the sequel by $(\bar{u}, \bar{y})$, and that the latter satisfies, with $\bar{p}$ and $\bar{\eta}$ its associated multipliers, the following assumptions:

(A2) The control $\bar{u}$ is continuous over $[0, T]$, and there exists $\alpha>0$ such that

$$
H_{u u}\left(\bar{u}(t), \bar{y}(t), \bar{p}\left(t^{ \pm}\right)\right) \geq \alpha, \quad \text { for all } t \in[0, T]
$$

(A3) Uniform regularity of the state constraint near the contact set, i.e., there exists $\beta, \varepsilon>0$ such that

$$
\left|g_{u}^{(1)}(\bar{u}(t), \bar{y}(t))\right| \geq \beta, \quad \text { for a.a. } t, \operatorname{dist}\{t ; I(g(\bar{y}))\} \leq \varepsilon
$$

A condition stronger than (A2) implying the continuity of the control is the uniform strong convexity of the Hamiltonian w.r.t. the control variable, i.e. there exists $\alpha>0$, such that

$$
H_{u u}\left(\hat{u}, \bar{y}(t), \bar{p}\left(t^{ \pm}\right)\right) \geq \alpha, \quad \text { for all } \hat{u} \in \mathbb{R} \text { and all } t \in[0, T]
$$

It is well-know (see e.g. $[15,17])$ that when (A2)-(A3) hold, then $\bar{u}$ and the multiplier $\bar{\eta}$ are Lipschitz continuous. In particular this implies that all touch points $\tau_{\text {to }}$ are nonessential, i.e. $\left[\bar{\eta}\left(\tau_{\text {to }}\right)\right]=0$. Furthermore, (A3) implies that (1.17) holds, and that the multipliers $(\bar{p}, \bar{\eta})$ associated with $(\bar{u}, \bar{y})$ are unique. This is a consequence of the lemma below. For $\delta>0$, let $\Omega^{\delta}:=\{t \in[0, T]$, dist $\{t ; I(g(\bar{y}))\}<\delta\}$.

Lemma 1.2. Assumption (A3) implies that for all $0<\delta<\varepsilon$, with the $\varepsilon$ of (1.21), assumed to be so small that $\Omega^{\varepsilon} \subset[a, T]$ for some $a>0$, the linear mapping

$$
\mathcal{U} \rightarrow W^{1, \infty}\left(\Omega^{\delta}\right),\left.\quad v \mapsto(D G(\bar{u}) v)\right|_{\Omega^{\delta}},
$$

where $\left.\right|_{\Omega^{\delta}}$ denotes the restriction to the set $\Omega^{\delta}$, is onto.

Proof. Let us recall the proof of [7], Lemma 9. For $v \in \mathcal{U}$, we have that $D G(\bar{u}) v=g_{y}(\bar{y}) z_{v}$, where $z_{v}$ is the (unique) solution in $\mathcal{Y}$ of the linearized state equation:

$$
\dot{z}_{v}=f_{u}(\bar{u}, \bar{y}) v+f_{y}(\bar{u}, \bar{y}) z_{v}, \quad \text { a.e. on }[0, T], \quad z_{v}(0)=0 .
$$

It is easy to see that

$$
\frac{\mathrm{d}}{\mathrm{d} t} g_{y}(\bar{y}(t)) z_{v}(t)=g_{u}^{(1)}(\bar{u}, \bar{y}) v+g_{y}^{(1)}(\bar{u}, \bar{y}) z_{v}
$$

and since by $(1.21)$ and $(\mathrm{A} 1), g_{u}^{(1)}(\bar{u}, \bar{y})$ is uniformly invertible on a neighborhood of $\Omega^{\delta}$ for small $\delta>0$, the result follows as a consequence of Gronwall's lemma.

We will also make in addition to (A2)-(A3) the following assumptions:

(A4) The trajectory $(\bar{u}, \bar{y})$ has a finite set of junction times $\overline{\mathcal{T}}$, and we assume that $g(\bar{y}(T))<0$.

(A5) Uniform strict complementarity on boundary arcs:

$$
\exists \beta>0 \quad \frac{\mathrm{d} \bar{\eta}}{\mathrm{d} t}(t) \geq \beta \quad \text { for all } t \text { in the interior of boundary arcs. }
$$

(A6) Non tangentiality at second-order at (nonessential) touch points: for all touch point $\bar{\tau}_{\text {to }}$,

$$
\left.\frac{\mathrm{d}^{2}}{\mathrm{~d} t^{2}} g(\bar{y}(t))\right|_{t=\bar{\tau}_{\text {to }}}<0
$$


Note that (1.26) makes sense, since $\frac{\mathrm{d}^{2}}{\mathrm{~d} t^{2}} g(\bar{y}(t))$ is by (1.6) a continuous function of $(\bar{y}, \bar{u}, \dot{\bar{u}})$, and $\bar{u}$ and $\dot{\bar{u}}$ are continuous at a touch point $\bar{\tau}_{\text {to }}$ (indeed, $\bar{\tau}_{\text {to }}$ being a nonessential touch point, $\left(\bar{\tau}_{\text {to }}-\varepsilon, \bar{\tau}_{\text {to }}+\varepsilon\right) \cap \operatorname{supp}(\mathrm{d} \bar{\eta})=\emptyset$ for some small $\varepsilon>0$, so the continuity of $\dot{\bar{u}}$ follows from the implicit function theorem applied to the relation $\left.H_{u}(\bar{u}, \bar{y}, \bar{p})=0\right)$. This condition is similar to the reducibility hypothesis when the state constraint is of order $q \geq 2$ (see [6]). The lemma below will be proved later (see Lem. 4.9), the proof being based on the alternative formulation (Def. 4.1).

Lemma 1.3. Let $(\bar{u}, \bar{y})$ be a stationary point of $(\mathcal{P})$ satisfying $(\mathrm{A} 2)-(\mathrm{A} 4)$. Then assumption (A5) implies that the following non-tangentiality condition at second-order holds at entry and exit points:

$$
\left.\frac{\mathrm{d}^{2}}{\mathrm{~d} t^{2}} g(\bar{y}(t))\right|_{t=\bar{\tau}_{\text {en }}^{-}}<0, \quad \bar{\tau}_{\text {en }} \in \overline{\mathcal{T}}_{\text {en }} ;\left.\quad \frac{\mathrm{d}^{2}}{\mathrm{~d} t^{2}} g(\bar{y}(t))\right|_{t=\bar{\tau}_{\text {ex }}^{+}}<0, \quad \bar{\tau}_{\text {ex }} \in \overline{\mathcal{T}}_{\text {ex }}
$$

\section{Structural Stability OF Stationary POINTS}

Let $(\bar{u}, \bar{y})$ be a stationary point of $(\mathcal{P})$ satisfying $(\mathrm{A} 2)-(\mathrm{A} 6)$. Assume that $(\bar{u}, \bar{y})$ has $N_{\text {ba }}$ boundary arcs and $N_{\text {to }}$ touch points, and let $N:=N_{\text {ba }}+N_{\text {to }}$. Number the boundary arcs and touch points of $(\bar{u}, \bar{y})$ by $i=1, \ldots, N$, and denote by $I_{\mathrm{ba}}$ and $I_{\text {to }}$ the (disjoint) sets of index in $\{1, \ldots, N\}$ corresponding respectively to boundary arcs and touch points. Denote the junction times of $(\bar{u}, \bar{y})$ by $\overline{\mathcal{T}}_{\text {en }}=\left\{\bar{\tau}_{\text {en }}^{i}\right\}_{i \in I_{\text {ba }}}, \overline{\mathcal{T}}_{\text {ex }}=\left\{\bar{\tau}_{\text {ex }}^{i}\right\}_{i \in I_{\text {ba }}}$, and $\overline{\mathcal{T}}_{\text {to }}=\left\{\overline{\mathcal{T}}_{\text {to }}^{i}\right\}_{i \in I_{\text {to }}}$. For $\delta>0$, define

$$
\Omega_{i}^{\delta}:=\left(\bar{\tau}_{\mathrm{en}}^{i}-\delta, \bar{\tau}_{\mathrm{ex}}^{i}+\delta\right), \quad i \in I_{\mathrm{ba}}, \quad \Omega_{i}^{\delta}:=\left(\bar{\tau}_{\mathrm{to}}^{i}-\delta, \bar{\tau}_{\mathrm{to}}^{i}+\delta\right), \quad i \in I_{\mathrm{to}} .
$$

In view of $(\mathrm{A} 4),(\mathrm{A} 6)$ and $(1.27)$, we may fix $\kappa, \bar{\delta}>0$ satisfying the conditions below:

$\bar{\delta} \leq \varepsilon$ with the $\varepsilon$ of $(1.21)$,

$\frac{\mathrm{d}^{2}}{\mathrm{~d} t^{2}} g(\bar{y}(t)) \leq-\kappa<0 \quad$ on $\Omega_{i}^{\bar{\delta}} \backslash\left[\bar{\tau}_{\mathrm{en}}^{i}, \bar{\tau}_{\mathrm{ex}}^{i}\right]$, for all $i \in I_{\mathrm{ba}}$ and on $\Omega_{i}^{\bar{\delta}}$, for all $i \in I_{\mathrm{to}}$,

the sets $\left(\Omega_{i}^{\bar{\delta}}\right)_{1 \leq i \leq N}$ are pairwise disjoint and contained in $[a, T]$ for some $a>0$.

The next theorem gives a direct result (i.e. without using a shooting formulation) of the stability of structure of stationary points, when assumptions (A2)-(A6) are satisfied.

Theorem 2.1. Let $(\bar{u}, \bar{y})$ be a stationary point of $\left(\mathcal{P}^{\mu_{0}}\right)$ satisfying (A2)-(A6), and let $\bar{\delta}$ satisfy (2.2)-(2.4). Then for all $0<\delta<\bar{\delta}$ and all stable extensions $\left(\mathcal{P}^{\mu}\right)$ of $\left(\mathcal{P}^{\mu_{0}}\right)$, there exists a neighborhood $V_{u} \times V_{\mu}$ of $\left(\bar{u}, \mu_{0}\right)$ in $\mathcal{U} \times M$, such that all stationary points $(u, y)$ of $\left(\mathcal{P}^{\mu}\right)$ with $(u, \mu) \in V_{u} \times V_{\mu}$ satisfy the following properties, with the contact set $I\left(g^{\mu}(y)\right)$ defined by (1.9):

(S1) $I\left(g^{\mu}(y)\right) \subset \cup_{i=1}^{N} \Omega_{i}^{\delta}$;

(S2) for all $i \in I_{\mathrm{ba}}, I\left(g^{\mu}(y)\right) \cap \Omega_{i}^{\delta}$ is an interval of positive measure;

(S3) for all $i \in I_{\mathrm{to}}, I\left(g^{\mu}(y)\right) \cap \Omega_{i}^{\delta}$ is either empty, or a singleton, or an interval of positive measure.

When $(\mathrm{S} 1)-(\mathrm{S} 3)$ are satisfied, we say that a stationary point $(u, y)$ of $\left(\mathcal{P}^{\mu}\right)$ has a neighboring structure to that of $(\bar{u}, \bar{y})$.

Remark 2.2. We can actually state a "local" version of Theorem 2.1. More precisely, if a stationary point $(\bar{u}, \bar{y})$ of $\left(\mathcal{P}^{\mu_{0}}\right)$ satisfying (A3) has a boundary arc $\left[\bar{\tau}_{\text {en }}, \bar{\tau}_{\text {ex }}\right]$ (resp. a touch point $\bar{\tau}_{\text {to }}$ ) and if assumptions (A2) and (A4)-(A6) hold locally over $\left(\bar{\tau}_{\text {en }}-\delta, \bar{\tau}_{\text {ex }}+\delta\right)$ (resp. over $\left.\left(\bar{\tau}_{\text {to }}-\delta, \bar{\tau}_{\text {to }}+\delta\right)\right)$ for some $\delta>0$, then all stationary points $(u, y)$ of $\left(\mathcal{P}^{\mu}\right)$ with $(u, \mu)$ in the neighborhood of $\left(\bar{u}, \mu_{0}\right)$ have exactly one boundary arc on $\left(\bar{\tau}_{\mathrm{en}}-\delta, \bar{\tau}_{\text {ex }}+\delta\right)$ (resp. have at most either one touch point or one boundary arc on $\left(\bar{\tau}_{\text {to }}-\delta, \bar{\tau}_{\text {to }}+\delta\right)$ ).

The proof of Theorem 2.1 is given in Section 9 and will use two lemmas below. Note that by continuity of the mapping $(u, \mu) \mapsto g^{\mu}\left(y_{u}^{\mu}\right)$, it is immediate that all stationary points of a stable extension $\left(\mathcal{P}^{\mu}\right)$ with $(u, \mu)$ 
in the neighborhood of $\left(\bar{u}, \mu_{0}\right)$ satisfy (S1). Let us first define alternative multipliers needed in Lemma 2.3 (see also $[12,15,17,21,23]$ where these multipliers are used)

$$
\begin{aligned}
\eta^{1}(t) & :=\int_{t}^{T} \mathrm{~d} \eta(s)=-\eta\left(t^{+}\right) \\
p^{1}(t) & :=p(t)-\eta^{1}(t) g_{y}^{\mu}(y(t)) .
\end{aligned}
$$

With this definition, and without any assumptions on the arc structure of the trajectory (i.e. without assuming a finite number of junction points), we have that

$$
-\mathrm{d} p^{1}=\left(H_{y}^{\mu}\left(u, y, p^{1}\right)+\left(g^{\mu}\right)_{y}^{(1)}(u, y) \eta^{1}\right) \mathrm{d} t,
$$

and hence, the new alternative costate $p^{1}$ is absolutely continuous. Consequently, an equivalent form of (1.13)(1.14) (when $\alpha=1$ ) and (1.18) is, a.e. on $[0, T]$ :

$$
\begin{aligned}
-\dot{p}^{1}(t) & =H_{y}^{\mu}\left(u(t), y(t), p^{1}(t)\right)+\left(g^{\mu}\right)_{y}^{(1)}(u(t), y(t)) \eta^{1}(t), \quad p^{1}(T)=\phi_{y}^{\mu}(y(T)) \\
0 & =H_{u}^{\mu}\left(u(t), y(t), p^{1}(t)\right)+\left(g^{\mu}\right)_{u}^{(1)}(u(t), y(t)) \eta^{1}(t) .
\end{aligned}
$$

In addition, (1.16) implies the following (weaker) relations, since $\eta^{1}$ is constant on interior arcs:

$$
0=\left(g^{\mu}\right)^{(1)}(u(t), y(t)) \quad \text { on boundary arcs, } \quad 0=\dot{\eta}^{1}(t) \quad \text { on interior arcs. }
$$

Note that given a trajectory $(u, y)$ of a stable extension $\left(\mathcal{P}^{\mu}\right)$, if $(u, \mu)$ is close enough to $\left(\bar{u}, \mu_{0}\right)$, Robinson's constraint qualification (1.17) still holds. This implies the uniqueness of the multipliers associated with a stationary point $(u, y)$ of $\left(\mathcal{P}^{\mu}\right)$ with $(u, \mu)$ in the neighborhood of $\left(\bar{u}, \mu_{0}\right)$. The two lemmas below, used in the proof of Theorem 2.1, are proved in Section 9.

Lemma 2.3. Let $(\bar{u}, \bar{y})$ be a stationary point of $\left(\mathcal{P}^{\mu_{0}}\right)$ satisfying $(\mathrm{A} 2)-(\mathrm{A} 3)$ with multipliers $(\bar{p}, \bar{\eta})$, and let the associated alternative multipliers $\left(\bar{p}^{1}, \bar{\eta}^{1}\right)$ be given by (2.5)-(2.6). Consider a stable extension $\left(\mathcal{P}^{\mu}\right)$, and let $\left(u_{n}, y_{n}=y_{u_{n}}^{\mu_{n}}\right)$ be a stationary point of $\left(\mathcal{P}^{\mu_{n}}\right)$, such that $u_{n} \rightarrow \bar{u}$ in $L^{\infty}$ and $\mu_{n} \rightarrow \mu_{0}$. Denote by $p_{n}, \eta_{n}$ the (unique) multipliers associated with $\left(u_{n}, y_{n}\right)$, and let $p_{n}^{1}, \eta_{n}^{1}$ be given by $(2.5)-(2.6)$. Then:

(1) the sequence $\left(\mathrm{d} \eta_{n}\right)$ is bounded in $\mathcal{M}[0, T]$;

(2) $\left\|\mathrm{d} \eta_{n}-\mathrm{d} \bar{\eta}\right\|_{1, \infty *} \rightarrow 0$, where $\|\cdot\|_{1, \infty *}$ denote the norm of the dual of $W^{1, \infty}$ for the strong topology;

(3) $p_{n}^{1} \rightarrow \bar{p}^{1}$ uniformly over $[0, T]$;

(4) $\eta_{n}^{1} \rightarrow \bar{\eta}^{1}$ uniformly over $[0, T]$.

Remark 2.4. Note that under the assumptions of Lemma 2.3, by (2.6) and (2.5), we deduce the uniform convergence of $\left(p_{n}, \eta_{n}\right)$ towards $(\bar{p}, \bar{\eta})$.

The key tool for deriving the structural stability result of Theorem 2.1 is the following lemma.

Lemma 2.5. Let $(\bar{u}, \bar{y})$ be a stationary point of $\left(\mathcal{P}^{\mu_{0}}\right)$ satisfying $(\mathrm{A} 2)-(\mathrm{A} 6)$, and let $\bar{\delta}$ be defined as in Theorem 2.1. Then for all $0<\delta<\bar{\delta}$ and all stable extensions $\left(\mathcal{P}^{\mu}\right)$ of $\left(\mathcal{P}^{\mu_{0}}\right)$, there exists a neighborhood $V_{u} \times V_{\mu}$ of $\left(\bar{u}, \mu_{0}\right)$ in $\mathcal{U} \times M$, such that if $(u, y)$ is a stationary point of $\left(\mathcal{P}^{\mu}\right)$ with $(u, \mu) \in V_{u} \times V_{\mu}$, then $(u, y)$ has no interior arc contained in $\Omega_{i}^{\delta}$, for all $i=1, \ldots, N$. 


\section{Statement of the Main Result}

Let us first recall the second-order conditions of $[4,7]$. Let the linearized control and state spaces be respectively $\mathcal{V}:=L^{2}(0, T)$ and $\mathcal{Z}:=H^{1}\left(0, T ; \mathbb{R}^{n}\right)$, where $H^{1}(0, T)=W^{1,2}(0, T)$. The quadratic function over $\mathcal{V} \times \mathcal{Z}$ involved in the second-order conditions is:

$$
\mathcal{J}(v, z):=\int_{0}^{T} H_{(u, y),(u, y)}(\bar{u}, \bar{y}, \bar{p})((v, z),(v, z)) \mathrm{d} t+z(T)^{*} \phi_{y y}(\bar{y}(T)) z(T)+\int_{0}^{T} z(t)^{*} g_{y y}(\bar{y}(t)) z(t) \mathrm{d} \bar{\eta}(t)
$$

and the set of constraints (defining the critical cone):

$$
\begin{aligned}
\dot{z}=f_{u}(\bar{u}, \bar{y}) v+f_{y}(\bar{u}, \bar{y}) z & \text { on }[0, T], & & z(0)=0 \\
g_{y}(\bar{y}(t)) z(t) & =0 & & t \in \overline{\mathcal{I}}_{b} \\
g_{y}(\bar{y}(\tau)) z(\tau) & \leq 0 & & \tau \in \overline{\mathcal{T}}_{\text {to }},
\end{aligned}
$$

where $\overline{\mathcal{I}}_{b}$ and $\overline{\mathcal{T}}_{\text {to }}$ denote respectively the union of boundary arcs and the set of touch points of $(\bar{u}, \bar{y})$.

Theorem $3.1([4,7])$. (i) Let $(\bar{u}, \bar{y})$ be a local solution of $(\mathcal{P})$ satisfying $(\mathrm{A} 2)-(\mathrm{A} 5)$. Then

$$
\mathcal{J}(v, z) \geq 0, \quad \text { for all }(v, z) \in \mathcal{V} \times \mathcal{Z} \text { satisfying }(3.2)-(3.4)
$$

(ii) Let $(\bar{u}, \bar{y})$ be a stationary point of $(\mathcal{P})$ satisfying $(\mathrm{A} 2)-(\mathrm{A} 5)$. Then

$$
\mathcal{J}(v, z)>0, \quad \text { for all }(v, z) \in \mathcal{V} \times \mathcal{Z},(v, z) \neq 0, \text { satisfying }(3.2)-(3.4),
$$

iff $(\bar{u}, \bar{y})$ is a local solution of $(\mathcal{P})$ satisfying the quadratic growth condition:

$$
\exists c, \rho>0, \quad J(u) \geq J(\bar{u})+c\|u-\bar{u}\|_{2}^{2}, \quad \forall u \in \mathcal{U} ; G(u) \in K,\|u-\bar{u}\|_{\infty} \leq \rho .
$$

Let us recall that a quadratic form $Q$ on an Hilbert space $\mathcal{H}$ is a Legendre form, if $Q$ is weakly lower semicontinuous and if for all weakly convergent subsequence $\left(v_{n}\right) \in \mathcal{H}^{\mathbb{N}}$, say $v_{n} \rightarrow v$, we have that $v_{n} \rightarrow v$ strongly if $Q\left(v_{n}\right) \rightarrow Q(v)$. Using (A2) we can show that the quadratic form $\mathcal{J}$ is a Legendre form (see [8,18]). This plays a role to obtain the no-gap second-order conditions of Theorem 3.1.

In the stability and sensitivity analysis, we will use the condition below, stronger than (3.6):

$$
\mathcal{J}(v, z)>0, \quad \text { for all }(v, z) \in \mathcal{V} \times \mathcal{Z},(v, z) \neq 0, \text { satisfying }(3.2)-(3.3) .
$$

Definition 3.2. Let $(\bar{u}, \bar{y})=\left(u^{\mu_{0}}, y^{\mu_{0}}\right)$ be a stationary point of $\left(\mathcal{P}^{\mu_{0}}\right)$. We say that $(\bar{u}, \bar{y})$ satisfies the uniform quadratic growth condition, if for all stable extensions $\left(\mathcal{P}^{\mu}\right)$ of $\left(\mathcal{P}^{\mu_{0}}\right)$ satisfying (1.4), there exist $c, \rho>0$ and an open neighborhood $V_{0}$ of $\mu_{0}$, such that for all $\mu \in V_{0}$, there exists a unique stationary point $\left(u^{\mu}, y^{\mu}\right)$ of $\left(\mathcal{P}^{\mu}\right)$ with $\left\|u^{\mu}-\bar{u}\right\|_{\infty} \leq \rho$, and this point satisfies

$$
J^{\mu}(u) \geq J^{\mu}\left(u^{\mu}\right)+c\left\|u-u^{\mu}\right\|_{2}^{2}, \quad \forall u \in \mathcal{U} ; G^{\mu}(u) \in K,\|u-\bar{u}\|_{\infty} \leq \rho, \quad \forall \mu \in V_{0} .
$$

Of course (3.9) implies that $\left(u^{\mu}, y^{\mu}\right)$ is a local solution of $\left(\mathcal{P}^{\mu}\right)$. Note that the constants $c$ and $\rho$ in the uniform growth condition (3.9) does not depend on $\mu$.

The arc structure of the trajectory (in the sense of number and order of boundary arcs and touch points) is not necessarily stable under a small perturbation. However, by (A5), boundary arcs are locally preserved, and by (A6), the only three possibilities for a touch point is to become a boundary arc, remain a touch point or become inactive at a local solution of the perturbed problem, i.e. the solutions of the perturbed problems have a neighboring arc structure of active constraints to that of $(\bar{u}, \bar{y})$ (see Th. 2.1). Below is our main result (together with Ths. 2.1 and 6.1), that will be proved later in Section 5 . 
Theorem 3.3. Let $(\bar{u}, \bar{y})$ be a stationary point of $(\mathcal{P})$ satisfying $(\mathrm{A} 2)-(\mathrm{A} 6)$. Then assertions (i) and (ii) below are equivalent:

(i) The uniform quadratic growth (Def. 3.2) holds.

(ii) The strong second-order sufficient condition (3.8) holds.

If either point (i) or (ii) is satisfied, for $\mu \in V_{0}$ denote by $\left(u^{\mu}, y^{\mu}\right)$ the unique local solution of $\left(\mathcal{P}^{\mu}\right)$ with $\left\|u^{\mu}-\bar{u}\right\| \leq \rho$, and by $\left(p^{\mu}, \eta^{\mu}\right)$ the (unique) associated multipliers. Then $\left(u^{\mu}, y^{\mu}\right)$ has a neighboring structure to that of $(\bar{u}, \bar{y})$, and the mapping $\mu \mapsto\left(u^{\mu}, y^{\mu}, p^{\mu}, \eta^{\mu}\right) \in C^{0}[0, T] \times C^{1}\left([0, T] ; \mathbb{R}^{n}\right) \times C^{0}\left([0, T] ; \mathbb{R}^{n *}\right) \times C^{0}[0, T]$ is Lipschitz continuous on $V_{0}$.

The above result implies that the solutions of the perturbed problems satisfy the quadratic growth condition (3.9), and hence the no-gap sufficient condition (3.6) by Theorem 3.1(ii). The lemma below (proved at the end of Sect. 5) shows that the strong second-order sufficient condition (3.8) remains satisfied as well for the perturbed problems (this will be useful for the analysis of the homotopy algorithm in Sect. 8).

Lemma 3.4. Under assumptions (A2)-(A6), if either point (i) or (ii) of Theorem 3.3 is satisfied, then the locally unique stationary point $\left(u^{\mu}, y^{\mu}\right)$ of $\left(\mathcal{P}^{\mu}\right)$ satisfies the strong second-order sufficient condition (3.8), for $\mu$ close enough to $\mu_{0}$.

Remark 3.5. We show more precisely (see Lem. 5.1) that under assumptions (A2)-(A6) and point (i) or (ii) of Theorem 3.3, then the shooting parameters associated with $\left(u^{\mu}, y^{\mu}\right)$ (initial costate, jump parameters at entry times and all junction times, see the next section) are Lipschitz continuous functions of $\mu$.

Related results to Theorem 3.3, based on a shooting approach (see the next section) too, are [23], Theorem 8.3, where the existence of a locally unique local solution of $\left(\mathcal{P}^{\mu}\right)$ having the same structure as $(\bar{u}, \bar{y})$ was shown (but the uniqueness of the stationary point or the converse implication "(i) $\Rightarrow$ (ii)" are not discussed), and [6], Theorem 4.3, where only the uniqueness of stationary points satisfying some restrictions on the arc structure is argued. In addition, both results assume the absence of touch points for state constraints of first-order. Here we are able to show that $\left(u^{\mu}, y^{\mu}\right)$ is locally the unique stationary point of $\left(\mathcal{P}^{\mu}\right)$ (see Lem. 5.4) thanks to the analysis done in Section 2. As mentioned in the Introduction, this is difficult to compare to $[12,21,22]$ where an infinite dimensional approach was used, which required weaker assumptions, e.g. (A4)-(A6) are not needed, so their results are more general than Theorem 3.3, but the conclusions obtained are also weaker than those of Theorem 3.3.

In Section 6, we will provide the first-order expansion of the local optimal solution and associated multipliers of the perturbed problem (see Th. 6.1).

\section{Alternative And Shooting Formulations}

\subsection{Alternative formulation of optimality conditions}

In presence of pure state constraints, a reformulation of the optimality conditions is needed to apply shooting methods. Our results are based on the following alternative formulation of optimality conditions, see e.g. $[6,9,17,19,25]$. We use in this alternative formulation another set of alternative multipliers, that we denote by $\left(p_{1}, \eta_{1}\right)$, different from the alternative multipliers $\left(p^{1}, \eta^{1}\right)$ used in Section 2 . Whereas the latter are continuous, $\left(p_{1}, \eta_{1}\right)$ have jumps at entry points. The jumps of $p_{1}$ at entry times $\tau_{\text {en }}$, denoted by $\nu_{\tau_{\text {en }}}^{1}$, are part of the shooting parameters used in the shooting algorithm.

Definition 4.1. A trajectory $(u, y)$ is solution of the alternative formulation, if it has finitely many junction times $\mathcal{T}$ and $g^{\mu}(y(T))<0$, if $(u, y) \in P C_{\mathcal{T}}^{0}[0, T] \times P C_{\mathcal{T}}^{1,0}\left([0, T] ; \mathbb{R}^{n}\right)$ and if there exist $p_{1} \in P C_{\mathcal{T}}^{1}\left([0, T] ; \mathbb{R}^{n *}\right)$, $\eta_{1} \in P C_{\mathcal{T}}^{1}[0, T]$, and alternative jump parameters $\nu_{\mathcal{T}_{\text {en }}}^{1}$ and $\nu_{\mathcal{T}_{\text {to }}}$, such that the following relations are satisfied, 
with the augmented Hamiltonian (1.8) (time dependence is omitted):

$$
\begin{array}{rlrl}
\dot{y} & =f^{\mu}(u, y) \quad \text { on }[0, T], \quad y(0)=y_{0}^{\mu} \\
-\dot{p}_{1} & =\tilde{H}_{y}^{\mu}\left(u, y, p_{1}, \eta_{1}\right) \quad \text { on }[0, T] \backslash \mathcal{T} \\
0 & =\tilde{H}_{u}^{\mu}\left(u, y, p_{1}, \eta_{1}\right) & & \text { on }[0, T] \backslash \mathcal{T} \\
\left(g^{\mu}\right)^{(1)}(u, y) & =0 \quad \text { on } \mathcal{I}_{b} & & \\
\eta_{1}(t) & =0 \quad \text { on }[0, T] \backslash \mathcal{I}_{b} & & \\
p_{1}(T) & =\phi_{y}^{\mu}(y(T)) & & \\
g^{\mu}\left(y\left(\tau_{\text {en }}\right)\right) & =0, & & \\
g^{\mu}\left(y\left(\tau_{\text {to }}\right)\right) & =0, \quad \tau_{\text {to }} \in \mathcal{T}_{\text {en }} & & \\
{\left[p_{1}\left(\tau_{\text {en }}\right)\right]} & =-\nu_{\tau_{\text {en }}}^{1} g_{y}^{\mu}\left(y\left(\tau_{\text {en }}\right)\right), & & \tau_{\text {en }} \in \mathcal{T}_{\text {en }} \\
{\left[p_{1}\left(\tau_{\text {ex }}\right)\right]} & =0, & & \\
{\left[p_{1}\left(\tau_{\text {to }}\right)\right]} & =-\nu_{\tau_{\text {to }}} g_{y}^{\mu}\left(y\left(\tau_{\text {to }}\right)\right), & & \tau_{\text {to }} \in \mathcal{T}_{\text {to }} .
\end{array}
$$

A solution of the alternative formulation satisfies the additional conditions, if the conditions below hold:

$$
\begin{aligned}
g^{\mu}(y(t)) & <0 \quad \text { on }[0, T] \backslash\left(\mathcal{I}_{b} \cup \mathcal{T}_{\text {to }}\right) \\
\dot{\eta}_{1}(t) & \leq 0 \quad \text { on } \operatorname{Int} \mathcal{I}_{b} \\
\nu_{\tau_{\text {en }}}^{1} & =\eta_{1}\left(\tau_{\text {en }}^{+}\right), \quad \tau_{\text {en }} \in \mathcal{T}_{\text {en }} ; \quad \eta_{1}\left(\tau_{\text {ex }}^{-}\right)=0, \quad \tau_{\text {ex }} \in \mathcal{T}_{\text {ex }} \\
\nu_{\tau_{\text {to }}} & =0, \quad \tau \in \mathcal{T}_{\text {to }} .
\end{aligned}
$$

Proposition 4.2 (see e.g. $[17,19,28])$. Let $(\bar{u}, \bar{y})$ be a local solution of $(\mathcal{P})$, satisfying $(\mathrm{A} 2)-(\mathrm{A} 4)$. Then $(\bar{u}, \bar{y})$ is solution of alternative formulation (4.1)-(4.11), and satisfies additional conditions (4.12)-(4.15).

The following remarks comment on those optimality conditions and on the relations existing between the different sets of multipliers.

Remark 4.3. It can be shown (see [6], Prop. 2.10) that under assumptions (1.22) (resp. (A2)) and (A3)-(A4), relations (4.1)-(4.15) characterize regular $(\alpha=1)$ Pontryagin extremals (resp. stationary points), and the (unique) classical multipliers $\mathrm{d} \eta \in \mathcal{M}_{+}[0, T]$ and $p \in B V\left([0, T] ; \mathbb{R}^{n *}\right)$ of Definition 1.1 are given by (recall that we adopted the convention $\left.\eta\left(T^{+}\right)=0\right)$ :

$$
\eta(t)=-\sum_{\tau \in \mathcal{T}_{\text {en }}} \nu_{\tau}^{1} \mathbf{1}_{[0, \tau)}(t)-\eta_{1}\left(t^{+}\right), \quad p(t)=p_{1}(t)+\eta_{1}(t) g_{y}^{\mu}(y(t)),
$$

with $\mathbf{1}_{[0, \tau)}(t)=1$ if $0 \leq t<\tau$ and zero otherwise. Equivalently, $\eta$ is given by $\mathrm{d} \eta(t)=-\dot{\eta}_{1}(t) \mathrm{d} t$.

The classical multipliers $(p, \eta)$ and alternative ones $\left(p_{1}, \eta_{1}\right)$ can be recovered from each other by (4.16) and (4.14). By (4.9)-(4.11) and additional conditions $(4.14)-(4.15)$, we have $(p, \eta) \in P C_{\mathcal{T}}^{1,0}\left([0, T] ; \mathbb{R}^{n *}\right) \times$ $P C_{\mathcal{T}}^{1,0}[0, T]$. It is also easy to see that, when $(4.16)$ holds, $\tilde{H}^{\mu}\left(\cdot, y, p_{1}, \eta_{1}\right)=H^{\mu}(\cdot, y, p)$, and hence, (1.20) is equivalent (with $\bar{p}_{1}$ and $\bar{\eta}_{1}$ the alternative multipliers associated with $\bar{u}$ ) to:

$$
\tilde{H}_{u u}\left(\bar{u}(t), \bar{y}(t), \bar{p}_{1}\left(t^{ \pm}\right), \bar{\eta}_{1}\left(t^{ \pm}\right)\right) \geq \alpha, \quad \text { for all } t \in[0, T]
$$

Remark 4.4. On $[0, T] \backslash \mathcal{T}$, the multipliers $\eta^{1}$ and $p^{1}$ in Section 2 are related to $p_{1}$ and $\eta_{1}$ by the following relations:

$$
\eta^{1}(t)=\sum_{\tau \in \mathcal{T}_{\text {en }}} \nu_{\tau}^{1} \mathbf{1}_{[0, \tau)}(t)+\eta_{1}(t), \quad p^{1}(t)=p_{1}(t)-\sum_{\tau \in \mathcal{T}_{\text {en }}} \nu_{\tau}^{1} \mathbf{1}_{[0, \tau)}(t) g_{y}^{\mu}(y(t)) .
$$


Remark 4.5. By (4.13)-(4.14), the following necessary condition holds:

$$
\nu_{\tau_{\mathrm{en}}}^{1} \geq 0, \quad \tau_{\mathrm{en}} \in \mathcal{T}_{\mathrm{en}} .
$$

Lemma 4.6. Let $(u, y)$ be a trajectory of $\left(\mathcal{P}^{\mu}\right)$ satisfying the alternative formulation. Assume that there exist $\alpha, \beta, \varepsilon>0$ such that (we denote here $\left.\left[u\left(t^{-}\right), u\left(t^{+}\right)\right]:=\left\{(1-\sigma) u\left(t^{-}\right)+\sigma u\left(t^{+}\right) ; \sigma \in[0,1]\right\}\right)$

$$
\begin{aligned}
& \alpha \leq \tilde{H}_{u u}^{\mu}\left(\hat{u}, y(t), p_{1}\left(t^{ \pm}\right), \eta_{1}\left(t^{ \pm}\right)\right) \quad \text { for all } \hat{u} \in\left[u\left(t^{-}\right), u\left(t^{+}\right)\right] \text {and all } t \in[0, T] \\
& \beta \leq\left|\left(g^{\mu}\right)_{u}^{(1)}(\hat{u}, y(t))\right| \quad \text { for all } \hat{u} \in\left[u\left(t^{-}\right), u\left(t^{+}\right)\right] \text {and all } t: \operatorname{dist}\left\{t ; I\left(g^{\mu}(y)\right)\right\} \leq \varepsilon .
\end{aligned}
$$

Then (4.14) is equivalent to the condition below

$$
\left(g^{\mu}\right)^{(1)}\left(u\left(\tau_{\text {en }}^{-}\right), y\left(\tau_{\text {en }}\right)\right)=0, \quad \tau_{\text {en }} \in \mathcal{T}_{\text {en }} ; \quad\left(g^{\mu}\right)^{(1)}\left(u\left(\tau_{\text {ex }}^{+}\right), y\left(\tau_{\text {ex }}\right)\right)=0, \quad \tau_{\text {ex }} \in \mathcal{T}_{\text {ex }} .
$$

Also (4.14) or (4.22) is equivalent to the continuity of the control at entry/exit points.

Proof. We recall here the proof (see [23] and [6], Prop. 2.15) since the arguments will be used later in Lemma 5.2. Since $\left(g^{\mu}\right)^{(1)}\left(u\left(\tau_{\mathrm{en}}^{+}\right), y\left(\tau_{\mathrm{en}}\right)\right)=0=\left(g^{\mu}\right)^{(1)}\left(u\left(\tau_{\mathrm{ex}}^{-}\right), y\left(\tau_{\mathrm{ex}}\right)\right)$, by $(4.21)-(4.22)$ is equivalent to the continuity of the control at entry and exit times. Now let $\tau \in \mathcal{T}_{\text {en }}$. By (4.3) and (4.9),

$$
\begin{aligned}
\tilde{H}_{u}^{\mu}\left(u\left(\tau^{-}\right), y(\tau), p_{1}\left(\tau^{-}\right), \eta_{1}\left(\tau^{-}\right)\right) & =\tilde{H}_{u}^{\mu}\left(u\left(\tau^{+}\right), y(\tau), p_{1}\left(\tau^{+}\right), \eta_{1}\left(\tau^{+}\right)\right) \\
& =\tilde{H}_{u}^{\mu}\left(u\left(\tau^{+}\right), y(\tau), p_{1}\left(\tau^{-}\right), \eta_{1}\left(\tau^{+}\right)-\nu_{\tau}^{1}\right) .
\end{aligned}
$$

If (4.14) holds, then we obtain ( since $\left.\eta_{1}\left(\tau^{-}\right)=0\right)$

$$
\tilde{H}_{u}^{\mu}\left(u\left(\tau^{-}\right), y(\tau), p_{1}\left(\tau^{-}\right), \eta_{1}\left(\tau^{-}\right)\right)=\tilde{H}_{u}^{\mu}\left(u\left(\tau^{+}\right), y(\tau), p_{1}\left(\tau^{-}\right), \eta_{1}\left(\tau^{-}\right)\right),
$$

which implies by (4.20) that $u\left(\tau^{-}\right)=u\left(\tau^{+}\right)$. Conversely, if (4.22) holds, i.e. if $u$ is continuous at $\tau$, then we obtain

$$
\left(\eta_{1}\left(\tau^{+}\right)-\nu_{\tau}^{1}\right)\left(g^{\mu}\right)_{u}^{(1)}(u(\tau), y(\tau))=0 .
$$

Since by $(4.21),\left(g^{\mu}\right)_{u}^{(1)}(u, y) \neq 0$, we obtain the result. Similar arguments hold at exit points.

Remark 4.7. By (4.11) and (4.15), (4.3) and hypothesis (4.20), we can show similarly that a solution (u,y) of the alternative formulation and additional conditions satisfying (4.20)-(4.21) is such that $u$ is also continuous at touch points, and hence $(u, y) \in P C_{\mathcal{T}}^{1,0}[0, T] \times P C_{\mathcal{T}}^{2,1}\left([0, T] ; \mathbb{R}^{n}\right)$.

Remark 4.8. At a touch point $\tau_{\text {to }}$, the function $t \mapsto g^{\mu}(y(t))$ has a local isolated maximum, and a continuous derivative at $\tau_{\text {to }}$ (due to the continuity of $u$ ), hence the condition below is satisfied (compare to (4.22)):

$$
\left(g^{\mu}\right)^{(1)}\left(u\left(\tau_{\text {to }}\right), y\left(\tau_{\text {to }}\right)\right)=0, \quad \tau \in \mathcal{T}_{\text {to }} .
$$

The next lemma provides in particular a proof for Lemma 1.3.

Lemma 4.9. Let $(u, y)$ be a trajectory of $\left(\mathcal{P}^{\mu}\right)$ solution of the alternative formulation and additional conditions. Assume that there exist $\alpha, \beta, \varepsilon>0$ such that (4.20) and (4.21) holds. Then, for all $\tau_{\mathrm{en}} \in \mathcal{T}_{\mathrm{en}}$ and $\tau_{\mathrm{ex}} \in \mathcal{T}_{\mathrm{ex}}$,

$$
\left.\frac{\mathrm{d}^{2}}{\mathrm{~d} t^{2}} g^{\mu}(y(t))\right|_{t=\tau_{\text {en }}^{-}}<0 \quad \text { iff } \quad \dot{\eta}_{1}\left(\tau_{\text {en }}^{+}\right)<0 ;\left.\quad \frac{\mathrm{d}^{2}}{\mathrm{~d} t^{2}} g^{\mu}(y(t))\right|_{t=\tau_{\text {ex }}^{+}}<0 \quad \text { iff } \quad \dot{\eta}_{1}\left(\tau_{\text {ex }}^{-}\right)<0 .
$$

Proof. Let $\tau_{\text {en }} \in \mathcal{T}_{\text {en }}$. We omit in the proof the superscript ${ }^{\mu}$ on $\tilde{H}, g$ and $f$. Derivation w.r.t. time of the relation (4.3) on the left and right neighborhood of $\tau_{\text {en }}$ yields (omitting the dependence in $t$ and arguments $\left(u, y, p_{1}, \eta_{1}\right)$ of $\left.\tilde{H}\right)$ :

$$
\tilde{H}_{u u} \dot{u}+\tilde{H}_{u y} f(u, y)-\tilde{H}_{y} f_{u}(u, y)+g_{u}^{(1)}(u, y) \dot{\eta}_{1}=0
$$


Recall that $g^{(1)}(u, y)=g_{y}(y) f(u, y)$. By Lemma 4.6 and (4.14), $u$ is continuous, so it follows that, taking the jumps at time $\tau_{\mathrm{en}}$ (omitting again arguments and setting $\nu^{1}:=\nu_{\tau_{\mathrm{en}}}^{1}$ ):

$$
\begin{aligned}
{\left[\tilde{H}_{u u}\right] } & =\left[p_{1}\right] f_{u u}+\left[\eta_{1}\right] g_{u u}^{(1)}=-\nu^{1} g_{y} f_{u u}+\nu^{1} g_{u u}^{(1)}=0, \\
{\left[\tilde{H}_{u y}\right] f-\left[\tilde{H}_{y}\right] f_{u} } & =\left(\left[p_{1}\right] f_{u y}+\left[\eta_{1}\right] g_{u y}^{(1)}\right) f-\left(\left[p_{1}\right] f_{y}+\left[\eta_{1}\right] g_{y}^{(1)}\right) f_{u} \\
& =\left(-\nu^{1} g_{y} f_{u y}+\nu^{1} g_{u y}^{(1)}\right) f-\left(-\nu^{1} g_{y} f_{y}+\nu^{1} g_{y}^{(1)}\right) f_{u}=0 .
\end{aligned}
$$

Taking then the jump in (4.25) at time $\tau_{\mathrm{en}}$, the above relations imply that

$$
\tilde{H}_{u u}[\dot{u}]+g_{u}^{(1)}\left[\dot{\eta}_{1}\right]=0 .
$$

Since $u, y, p_{1}$ and $\eta_{1}$ are all continuous at exit times by Lemma 4.6, (4.26) holds as well at exit times. Since the function $\frac{\mathrm{d}^{2}}{\mathrm{~d} t^{2}} g(y(t))=g^{(2)}(\dot{u}, u, y)$, with $g^{(2)}$ given by (1.5), vanishes on $\left(\tau_{\mathrm{en}}, \tau_{\mathrm{ex}}\right)$, and $(u, y)$ is continuous, we have by (4.21) that $g^{(2)}(\dot{u}, u, y)$ is discontinuous at $\tau$ iff $\dot{u}$ is, and hence by (4.26) and (4.20)-(4.21) iff $\dot{\eta}_{1}$ is. Since $\dot{\eta}_{1}=0$ locally outside $\left(\tau_{\mathrm{en}}, \tau_{\mathrm{ex}}\right)$, and $\dot{\eta}_{1} \leq 0$ on $\left(\tau_{\mathrm{en}}, \tau_{\mathrm{ex}}\right)$ by $(4.13)$, the result follows.

Remark 4.10. We know by [6], Lemma 3.6, that we can express the quadratic cost $\mathcal{J}$, using $\left(\bar{p}_{1}, \bar{\eta}_{1}\right)$ defined by (4.16) instead of $(\bar{p}, \bar{\eta})$, over the space of linearized trajectories $(v, z)$ satisfying $(3.2)$, by $\mathcal{J}(v, z)=\mathcal{J}_{1}(v, z)$, with

$$
\begin{aligned}
\mathcal{J}_{1}(v, z):= & \int_{0}^{T} \tilde{H}_{(u, y),(u, y)}\left(\bar{u}, \bar{y}, \bar{p}_{1}, \bar{\eta}_{1}\right)((v, z),(v, z)) \mathrm{d} t \\
& +z(T)^{*} \phi_{y y}(\bar{y}(T)) z(T)+\sum_{\tau \in \overline{\mathcal{T}}_{\mathrm{en}}} \bar{\nu}_{\tau}^{1} z(\tau)^{*} g_{y y}(\bar{y}(\tau)) z(\tau),
\end{aligned}
$$

where $\tilde{H}$ is the augmented Hamiltonian (1.8), and the constraint (3.3) is equivalent to

$$
\begin{array}{cc}
g_{y}(\bar{y}(\tau)) z(\tau)=0, & \tau \in \overline{\mathcal{T}}_{\text {en }} \\
g_{(u, y)}^{(1)}(\bar{u}(t), \bar{y}(t))(v(t), z(t))=0, & t \in \overline{\mathcal{I}}_{b} .
\end{array}
$$

Remark 4.11. The second-order sufficient condition (3.8) used in the stability and sensitivity analysis, is equivalent by Remark 4.10 to

$$
\mathcal{J}_{1}(v, z)>0, \quad \text { for all }(v, z) \in \mathcal{V} \times \mathcal{Z},(v, z) \neq 0 \text {, satisfying (3.2) and (4.28)-(4.29). }
$$

This condition is weaker than the one in [23], where the entry-point constraint (4.28) is omitted. The authors present a numerical method, based on Riccati equations, allowing to check the coercivity of the quadratic form $\mathcal{J}_{1}$ over the subspace defined by (3.2) and (4.29), which is of interest in applications, while the verification of (3.8) or (4.30) in practice remains open.

\subsection{Shooting formulation with nonessential touch points}

By (A2)-(A4), applying the implicit function theorem to (4.3)-(4.5), we may express the algebraic variables $\left(u, \eta_{1}\right)$ on each arc as $C^{1}$ functions of the differential variables $\left(y, p_{1}\right)$. Denote by $\mathcal{F}_{b}^{\mu}$ and $\mathcal{F}_{i}^{\mu}$ the flows on $\left(y, p_{1}\right)$ obtained respectively on boundary and interior arcs, by eliminating the algebraic variables, and write $\left(y, p_{1}\right)(t)=\left(y(t), p_{1}(t)\right)$. On each arc $\left(t_{1}, t_{2}\right)$, we have that

$$
\left(y, p_{1}\right)\left(t_{2}^{-}\right)=\mathcal{F}_{a}^{\mu}\left(\left(y, p_{1}\right)\left(t_{1}^{+}\right), t_{2}-t_{1}\right)
$$

where $\mathcal{F}_{a}^{\mu}$ equals $\mathcal{F}_{b}^{\mu}$ for a boundary arc, and $\mathcal{F}_{i}^{\mu}$ for an interior arc. So we can (and this is precisely the idea of shooting methods) describe the alternative optimality system (4.1)-(4.11) as a sequence of applications 
of mappings $\mathcal{F}_{b}^{\mu}$ and $\mathcal{F}_{i}^{\mu}$, combined with junction conditions. Note that the mappings $\left(x, t_{1}, t_{2}\right) \rightarrow \mathcal{F}_{a}^{\mu}\left(x, t_{2}-t_{1}\right)$, $a=i, b$, are (locally) $C^{1}$ w.r.t. all arguments, and allow in particular $t_{2}-t_{1}$ to be nonpositive.

Now let us view a touch point as a boundary arc of zero length. This makes sense since, as we will see later, under a small perturbation, a touch point may switch into a boundary arc. So we have an entry point and an exit point, $\tau_{\text {en }}$ and $\tau_{\text {ex }}$, whose common value is the one of the touch point. The jump $\nu_{\tau_{\text {en }}}^{1}$ at entry point $\tau_{\text {en }}$ equals $\nu_{\tau_{\text {to }}}$ (i.e., zero). There is a zero jump of $p_{1}$ at the entry (and exit) time $\tau_{\mathrm{en}}$.

Assume that we have $N_{\text {ba }}$ boundary $\operatorname{arcs}$ and $N_{\text {to }}$ touch points. Let $N:=N_{\text {ba }}+N_{\text {to }}$. We have now $N$ entry and $N$ exit points. Denote by $t^{\text {en }}$ (resp. $t^{\text {ex }}$ ) the $N$ dimensional vector of entry (resp. exit) points, taken in the chronological order, and $\nu_{i}^{1}:=\nu_{t_{i}^{\text {en }}}^{1}$. We use the notation $t_{0}^{\mathrm{ex}}:=0$ and $t_{N+1}^{\mathrm{en}}:=T$. We may rewrite the alternative formulation as follows, taking into account the continuity of state and of costate at exit points:

$$
\begin{aligned}
\left(y, p_{1}\right)(0) & =\left(y_{0}^{\mu}, p_{0}\right) \\
\left(y, p_{1}\right)\left(t_{i}^{\mathrm{en}-}\right) & =\mathcal{F}_{i}^{\mu}\left(\left(y, p_{1}\right)\left(t_{i-1}^{\mathrm{ex}}\right), t_{i}^{\mathrm{en}}-t_{i-1}^{\mathrm{ex}}\right), \quad i=1, \ldots, N+1, \\
\left(y, p_{1}\right)\left(t_{i}^{\mathrm{ex}}\right) & =\mathcal{F}_{b}^{\mu}\left(\left(y, p_{1}\right)\left(t_{i}^{\mathrm{en}}\right), t_{i}^{\mathrm{ex}}-t_{i}^{\mathrm{en}}\right), \quad i=1, \ldots, N, \\
{\left[p_{1}\left(t_{i}^{\mathrm{en}}\right)\right] } & =-\nu_{i}^{1} g_{y}^{\mu}\left(y\left(t_{i}^{\mathrm{en}}\right)\right), \quad i=1, \ldots, N, \\
p_{1}(T) & =\phi_{y}^{\mu}(y(T)) \\
g^{\mu}\left(y\left(t_{i}^{\mathrm{en}}\right)\right) & =0, \quad i=1, \ldots, N,
\end{aligned}
$$

where $p_{0} \in \mathbb{R}^{n *}$ denotes the initial value of the costate.

We come now to the definition of the shooting mapping. Let $\Theta:=\mathbb{R}^{n} \times \mathbb{R}^{N} \times \mathbb{R}^{N} \times \mathbb{R}^{N}$ be the space of shooting parameters, of dimension $\bar{N}:=n+3 N$. A vector of shooting parameters is denoted by

$$
\theta=\left(p_{0}^{*}, \nu^{1}, t^{\mathrm{en}}, t^{\mathrm{ex}}\right) \in \Theta .
$$

The shooting mapping $F$ is defined over a neighborhood $V_{\theta} \times V_{\mu}$ of $\left(\theta_{0}, \mu_{0}\right)$ in $\mathbb{R}^{\bar{N}} \times M_{0}$ into $\mathbb{R}^{\bar{N}}$, by

$$
F(\theta, \mu)=\left(\begin{array}{c}
p_{1}(T)-\phi_{y}^{\mu}(y(T)) \\
g^{\mu}\left(y\left(t^{\mathrm{en}}\right)\right) \\
\left(g^{\mu}\right)^{(1)}\left(u\left(t^{\mathrm{en}-}\right), y\left(t^{\mathrm{en}}\right)\right) \\
\left(g^{\mu}\right)^{(1)}\left(u\left(t^{\mathrm{ex}+}\right), y\left(t^{\mathrm{ex}}\right)\right)
\end{array}\right),
$$

where the values of $\left(y, p_{1}, u\right)$ at times $t_{i}^{\text {en }}, t_{i}^{\text {ex } \pm}, T$ are given by (4.32)-(4.35), and where we used e.g. the notation

$$
\left(g^{\mu}\right)^{(1)}\left(u\left(t^{\mathrm{en}-}\right), y\left(t^{\mathrm{en}}\right)\right):=\left(\left(g^{\mu}\right)^{(1)}\left(u\left(t_{i}^{\mathrm{en}-}\right), y\left(t_{i}^{\mathrm{en}}\right)\right)\right)_{1 \leq i \leq N} \in \mathbb{R}^{N} .
$$

Being a composition of $C^{1}$ mappings, the shooting mapping is itself locally of class $C^{1}$.

Let $(\bar{u}, \bar{y})$ be a stationary point of $(\mathcal{P})$, satisfying $(\mathrm{A} 2)-(\mathrm{A} 4)$, with finite set of junction times $\overline{\mathcal{T}}$. Let $I_{\mathrm{ba}}$ and $I_{\text {to }}$ denote the (disjoint) sets of index in $\{1, \ldots, N\}$ corresponding respectively to boundary arcs and touch points of the trajectory $(\bar{u}, \bar{y})$. Split $F$ into two components:

$$
F(\theta, \mu)=\left(\Phi(\theta, \mu)^{*}, \Psi(\theta, \mu)^{*}\right)^{*}
$$


where $\Psi$ corresponds to the components $g^{\mu}\left(y\left(t_{i}^{\text {en }}\right)\right)$ for $i \in I_{\text {to }}$, denoted by the vector $g^{\mu}\left(y\left(t_{\text {to }}^{\text {en }}\right)\right) \in \mathbb{R}^{N_{\text {to }}}$. Denote similarly by $\nu_{\text {to }}^{1}$ the vector of components $\nu_{i}^{1}$, for $i \in I_{\text {to. }}$. Consider the following nonlinear complementarity problem, for $\mu$ close to $\mu_{0}$ :

$$
\text { Find } \theta \in \Theta \text { such that } \Phi(\theta, \mu)=0 \text { and } \Psi(\theta, \mu) \in N(\theta) \text {, }
$$

where

$$
N(\theta):= \begin{cases}\mathbb{R}_{-}^{N_{\text {to }}} \cap\left(\nu_{\mathrm{to}}^{1}\right)^{\perp} & \text { if } \nu_{\text {to }}^{1} \in \mathbb{R}_{+}^{N_{\text {to }}}, \\ \emptyset & \text { otherwise. }\end{cases}
$$

Note that by (4.36)-(4.37) and (4.22)-(4.23), $\theta_{0}:=\left(\bar{p}_{1}(0)^{*}, \bar{\nu}^{1}, \bar{t}^{\mathrm{en}}, \bar{t}^{\mathrm{ex}}\right)$ is solution of (4.40) for $\mu=\mu_{0}$, with $\bar{t}^{\text {en }}$ and $\bar{t}^{\text {ex }}$ the vectors of times in $\overline{\mathcal{T}}_{\text {en }} \cup \overline{\mathcal{T}}_{\text {to }}$ and $\overline{\mathcal{T}}_{\text {ex }} \cup \overline{\mathcal{T}}_{\text {to }}$ respectively, in increasing order, $\bar{\nu}_{i}^{1}=\bar{\nu}_{\bar{t}_{i}^{\text {en }}}^{1}$ if $i \in I_{\text {ba }}$, and $\bar{\nu}_{i}^{1}=0$ if $i \in I_{\text {to }}$.

It should be underlined that we allow, in formulation of problem (4.40), entry times to be greater than exit times. However, we will check in the next section, after having shown that (4.40) has a locally unique solution, that the constraint $\nu_{\text {to }}^{1} \geq 0$ in (4.40) (compare with (4.19)) is sufficient, with assumption (A6), to ensure locally for $\mu$ in the neighborhood of $\mu_{0}$ that the solution of (4.40) is such that $t_{i}^{\mathrm{en}} \leq t_{i}^{\mathrm{ex}}$ for all $i \in I_{\mathrm{to}}$. In addition, we will show that by (1.26), strict complementarity $\dot{\eta}_{1}<0$ holds on the boundary $\operatorname{arc}\left(t_{i}^{\mathrm{en}}, t_{i}^{\mathrm{ex}}\right)$ whenever $t_{i}^{\mathrm{en}}<t_{i}^{\mathrm{ex}}$.

As we will see, the formulation (4.40) is strongly related with the associated linear-quadratic tangent problem $\min _{(v, z) \in \mathcal{V} \times \mathcal{Z}} \mathcal{J}_{1}(v, z)$ subject to the equality constraints (3.2) and (4.28)-(4.29), and the inequality constraint (3.4).

Remark 4.12. When the state constraint is of higher order, under small perturbations, a nonessential touch point satisfying (1.26) cannot switch into a boundary arc, i.e. it either becomes inactive, remains nonessential, or becomes an essential touch point (with a nonzero jump of the costate), see [6].

\section{StABILITY ANALYSIS}

In problem (4.40), there are inequality constraints that cannot be reduced to equality ones since strict complementarity does not hold at touch points, and those inequality constraints introduce nonsmoothness. Therefore we cannot apply the classical implicit function theorem as it is done in [23]. Our stability analysis uses the notion of strong regularity, introduced by Robinson in [31], applied to the complementarity problem (4.40).

The point $\theta_{0}$ solution of (4.40) for $\mu=\mu_{0}$ is strongly regular, if there exist neighborhoods $\left(V_{\theta}^{\prime}, V_{\delta}\right)$ in $\mathbb{R}^{\bar{N}} \times \mathbb{R}^{\bar{N}}$

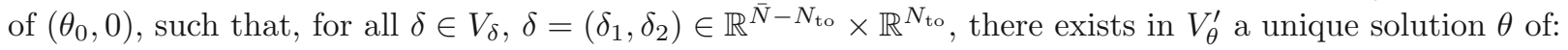

$$
\left\{\begin{array}{l}
D_{\theta} \Phi\left(\theta_{0}, \mu_{0}\right)\left(\theta-\theta_{0}\right)-\delta_{1}=0 \\
D_{\theta} \Psi\left(\theta_{0}, \mu_{0}\right)\left(\theta-\theta_{0}\right)-\delta_{2} \in N(\theta)
\end{array}\right.
$$

and the mapping $\Xi: \delta \rightarrow \theta(\delta)$ is Lipschitz continuous over $V_{\delta}$.

If $\theta_{0}$ is strongly regular, then by [31], there exist neighborhoods $\left(V_{\theta}, V_{\mu}\right)$ of $\left(\theta_{0}, \mu_{0}\right)$, such that for each $\mu \in V_{\mu}$, (4.40) has in $V_{\theta}$ a unique solution $\theta^{\mu}$,

$$
\theta^{\mu}=\left(p_{0}^{\mu *}, \nu^{\mu, 1}, t^{\mu, \mathrm{en}}, t^{\mu, \mathrm{ex}}\right),
$$

and there exists $\kappa>0$ such that for all $\mu, \mu^{\prime} \in V_{\mu}$,

$$
\left|\theta^{\mu}-\theta^{\mu^{\prime}}\right| \leq \kappa\left\|\mu-\mu^{\prime}\right\| .
$$

In addition, the following expansion of $\theta^{\mu}$ holds (see e.g. [8], p. 413, Eq. (5.41)):

$$
\theta^{\mu}=\Xi\left(-D_{\mu} F\left(\theta_{0}, \mu_{0}\right)\left(\mu-\mu_{0}\right)\right)+o\left(\left\|\mu-\mu_{0}\right\|\right) .
$$


Lemma 5.1. Under assumptions (A2)-(A6), (3.8) implies that $\theta_{0}$ is a strongly regular solution of (4.40) for $\mu=\mu_{0}$. More precisely, given $\delta=\left(\delta_{1}, \delta_{2}\right) \in \mathbb{R}^{\bar{N}-N_{\text {to }}} \times \mathbb{R}^{N_{\text {to }}}, \delta_{1}=\left(a_{T}, b_{\text {ba }}, c^{\text {en }}, c^{\text {ex }}\right) \in \mathbb{R}^{n} \times \mathbb{R}^{N_{\text {ba }}} \times \mathbb{R}^{N} \times \mathbb{R}^{N}$, $\delta_{2}=b_{\text {to }}$, there exists a unique $\omega \in \Theta, \omega=\left(\pi_{0}^{*}, \gamma^{1}, \sigma^{\mathrm{en}}, \sigma^{\mathrm{ex}}\right)$, solution of the following relation, equivalent to (5.1) with $\omega=\theta-\theta_{0}$ :

$$
\left\{\begin{array}{l}
D_{\theta} \Phi\left(\theta_{0}, \mu_{0}\right) \omega-\delta_{1}=0 \\
D_{\theta} \Psi\left(\theta_{0}, \mu_{0}\right) \omega-\delta_{2} \in N(\omega)
\end{array}\right.
$$

and $\omega$ is given as follows. Let $\left(v_{\delta}, z_{\delta}, \pi_{\delta}, \zeta_{\delta}, \lambda_{\delta}^{1}\right)$ be the unique solution and associated multipliers of the following linear-quadratic problem (recall that $\mathcal{J}_{1}$ is given by (4.27))

$$
\begin{aligned}
\left(\mathcal{P}^{\delta}\right) \quad \min _{(v, z) \in \mathcal{V} \times \mathcal{Z}} & \frac{1}{2} \mathcal{J}_{1}(v, z)+a_{T}^{*} z(T) \\
\text { subject to } \quad & (3.2),(4.29), \\
& g_{y}\left(\bar{y}\left(\bar{t}_{i}^{\mathrm{en}}\right)\right) z\left(\bar{t}_{i}^{\mathrm{en}}\right)=b_{i}, \quad i \in I_{\mathrm{ba}} \\
& g_{y}\left(\bar{y}\left(\bar{t}_{i}^{\text {en }}\right)\right) z\left(\bar{t}_{i}^{\text {en }}\right) \leq b_{i}, \quad i \in I_{\mathrm{to}},
\end{aligned}
$$

where the multipliers $\pi_{\delta}, \zeta_{\delta}$ and $\lambda_{\delta}^{1}$ are associated, respectively, with constraint (3.2), (4.29) and (5.7)-(5.8). Then $\omega$ is given by: $\pi_{0}=\pi_{\delta}(0), \gamma^{1}=\lambda_{\delta}^{1}$, and

$$
\begin{aligned}
\sigma_{i}^{\text {en }} & =\frac{c_{i}^{\text {en }}-g_{(u, y)}^{(1)}\left(\bar{u}\left(\bar{t}_{i}^{\text {en }}\right), \bar{y}\left(\bar{t}_{i}^{\text {en }}\right)\right)\left(v_{\delta}\left(\bar{t}_{i}^{\text {en }-}\right), z_{\delta}\left(\bar{t}_{i}^{\text {en }}\right)\right)}{\left.\frac{\mathrm{d}}{\mathrm{d} t} g^{(1)}(\bar{u}, \bar{y})\right|_{t=\bar{t}_{i}^{\text {en }}-}}, \quad i=1, \ldots, N, \\
\sigma_{i}^{\text {ex }} & =\frac{c_{i}^{\text {ex }}-g_{(u, y)}^{(1)}\left(\bar{u}\left(\bar{t}_{i}^{\text {ex }}\right), \bar{y}\left(\bar{t}_{i}^{\text {ex }}\right)\right)\left(v_{\delta}\left(\bar{t}_{i}^{\text {ex }}\right), z_{\delta}\left(\bar{t}_{i}^{\text {ex }}\right)\right)}{\left.\frac{\mathrm{d}}{\mathrm{d} t} g^{(1)}(\bar{u}, \bar{y})\right|_{t=\bar{t}_{i}^{\text {ex }}+}}, \quad i=1, \ldots, N .
\end{aligned}
$$

Proof. The proof uses the block-decoupling property of the Jacobian of the shooting mapping w.r.t. junction times for first-order state constraints established in [23], Lemma 4.2. See also [6], Lemma 4.5. Let us first explicit the relation (5.5). Let $(v, z, \pi, \zeta)$ be the linearized control, state, costate and state constraint multiplier solution of the linearized shooting equations (4.32)-(4.35):

$$
\begin{aligned}
\left(z, \pi_{1}\right)(0) & =\left(0, \pi_{0}\right) \\
\left(z, \pi_{1}\right)\left(\bar{t}_{i}^{\mathrm{en}-}\right) & =D \mathcal{F}_{i}^{\mu_{0}}\left(\left(\bar{y}, \bar{p}_{1}\right)\left(\bar{t}_{i-1}^{\mathrm{ex}}\right), \bar{t}_{i}^{\mathrm{en}}-\bar{t}_{i-1}^{\mathrm{ex}}\right)\left(z, \pi_{1}\right)\left(\bar{t}_{i-1}^{\mathrm{ex}}\right), \quad i=1, \ldots, N+1, \\
\left(z, \pi_{1}\right)\left(\bar{t}_{i}^{\mathrm{ex}}\right) & =D \mathcal{F}_{b}^{\mu_{0}}\left(\left(\bar{y}, \bar{p}_{1}\right)\left(\bar{t}_{i}^{\mathrm{en}+}\right), \bar{t}_{i}^{\mathrm{ex}}-\bar{t}_{i}^{\mathrm{en}}\right)\left(z, \pi_{1}\right)\left(\bar{t}_{i}^{\mathrm{en}+}\right), \quad i=1, \ldots, N, \\
{\left[\pi_{1}\left(\bar{t}_{i}^{\mathrm{en}}\right)\right] } & =-\bar{\nu}_{i}^{1} g_{y y}\left(\bar{y}\left(\bar{t}_{i}^{\mathrm{en}}\right)\right) z\left(\bar{t}_{i}^{\mathrm{en}}\right)-\gamma_{i}^{1} g_{y}\left(\bar{y}\left(\bar{t}_{i}^{\mathrm{en}}\right)\right), \quad i=1, \ldots, N .
\end{aligned}
$$

Then (5.5) writes

$$
\begin{aligned}
& \pi_{1}(T)=\phi_{y y}(\bar{y}(T)) z(T)+a_{T} \\
& g_{y}\left(\bar{y}\left(\bar{t}_{i}^{\text {en }}\right)\right) z\left(\bar{t}_{i}^{\text {en }}\right)=b_{i}, \quad i \in I_{\mathrm{ba}} \\
& g_{y}\left(\bar{y}\left(\bar{t}_{i}^{\text {en }}\right)\right) z\left(\bar{t}_{i}^{\text {en }}\right) \leq b_{i}, \quad \gamma_{i}^{1} \geq 0, \quad\left(g_{y}\left(\bar{y}\left(\bar{t}_{i}^{\text {en }}\right)\right) z\left(\bar{t}_{i}^{\text {en }}\right)-b_{i}\right) \gamma_{i}^{1}=0, \quad i \in I_{\text {to }} \\
& D g^{(1)}\left(\bar{u}\left(\bar{t}_{i}^{\text {en }}\right), \bar{y}\left(\bar{t}_{i}^{\text {en }}\right)\right)\left(v\left(\bar{t}_{i}^{\text {en }-}\right), z\left(\bar{t}_{i}^{\text {en }}\right)\right)+\left.\sigma_{i}^{\text {en }} \frac{\mathrm{d}}{\mathrm{d} t} g^{(1)}(\bar{u}, \bar{y})\right|_{t=\bar{t}_{i}^{\text {en }-}}=0, \quad i=1, \ldots, N \\
& D g^{(1)}\left(\bar{u}\left(\bar{t}_{i}^{\text {ex }}\right), \bar{y}\left(\bar{t}_{i}^{\text {ex }}\right)\right)\left(v\left(\bar{t}_{i}^{\text {ex }}\right), z\left(\bar{t}_{i}^{\text {ex }}\right)\right)+\left.\sigma_{i}^{\text {ex }} \frac{\mathrm{d}}{\mathrm{d} t} g^{(1)}(\bar{u}, \bar{y})\right|_{t=\bar{t}_{i}^{\text {ex }+}}=0, \quad i=1, \ldots, N .
\end{aligned}
$$

We recognize that (5.11)-(5.17) is the first-order optimality condition of problem $\left(\mathcal{P}_{\delta}\right)$, with $\gamma_{i}^{1}$ the multipliers associated with the constraints (5.7) and (5.8) for $i$ in respectively $I_{\mathrm{ba}}$ and $I_{\mathrm{to}}$. By (A2), we can show that the quadratic form $\mathcal{J}_{1}$ is a Legendre form over the space of linearized trajectories $(v, z)$ satisfying (3.2). Therefore, (3.8), equivalent to (4.30) by Remark 4.11, implies that $\mathcal{J}_{1}$ is uniformly positive over the linear space 
of $(v, z) \in \mathcal{V} \times \mathcal{Z}$ satisfying (3.2) and (4.28)-(4.29) (i.e. there exists $\alpha>0$ such that $\mathcal{J}_{1}(v, z) \geq \alpha\left(\|v\|_{\mathcal{V}}^{2}+\|z\|_{\mathcal{Z}}^{2}\right)$ for all $(v, z) \in \mathcal{V} \times \mathcal{Z}$ satisfying (3.2) and (4.28)-(4.29)). It follows then that problem ( $\left.\mathcal{P}^{\delta}\right)$ has, for all $\delta \in \mathbb{R}^{N}$, a unique solution and multipliers $\left(v_{\delta}, z_{\delta}, \pi_{\delta}, \zeta_{\delta}, \lambda_{\delta}^{1}\right)$ that are Lipschitz continuous w.r.t. $\delta$. Thus (5.5) has a unique solution, and by (5.18)-(5.19) and (A6) and (1.27), the variations of junction times $\sigma_{i}^{\text {en }}$ and $\sigma_{i}^{\text {ex }}$ are given by $(5.9)-(5.10)$.

Lemma 5.2. Under assumptions (A2)-(A6) and (3.8), there exists a neighborhood $V_{\mu}$ of $\mu_{0}$, such that the locally unique solution $\theta^{\mu}$ of (4.40) given by (5.2) satisfies:

$$
t_{i}^{\mu, \mathrm{ex}} \geq t_{i}^{\mu, \mathrm{en}}, \quad \text { for all } i \in I_{\mathrm{to}}
$$

and

$$
t_{i}^{\mu, \mathrm{ex}}=t_{i}^{\mu, \mathrm{en}} \Leftrightarrow \nu_{i}^{\mu, 1}=0, \quad i \in I_{\mathrm{to}} .
$$

In particular, the solution $\left(u^{\mu}, y^{\mu}, p_{1}^{\mu}, \eta_{1}^{\mu}\right)$ of $(4.32)-(4.35)$ with $\theta=\theta^{\mu}$ is well-defined over $[0, T]$, and there exists a constant $\gamma>0$, such that for all $i \in I_{\text {to }}$ and all $\mu \in V_{\mu}$ :

$$
\dot{\eta}_{1}^{\mu}(t)<-\gamma \quad \text { on } \quad\left[t_{i}^{\mu, \mathrm{en}}, t_{i}^{\mu, \mathrm{ex}}\right] \quad \text { whenever } t_{i}^{\mu, \mathrm{ex}}>t_{i}^{\mu, \mathrm{en}} .
$$

Proof. Let $i \in I_{\mathrm{to}}$. By strong regularity (Lem. 5.1), we have that

$$
t_{i}^{\mu, \text { ex }}-t_{i}^{\mu, \text { en }}=\mathcal{O}\left(\left\|\mu-\mu_{0}\right\|\right), \quad \nu_{i}^{\mu, 1}=\mathcal{O}\left(\left\|\mu-\mu_{0}\right\|\right) .
$$

Denote by $\left(u, y, p_{1}, \eta_{1}\right)$ the solution of (4.32)-(4.35) for $\theta=\theta^{\mu}$. Note that this is well-defined on each arc, but not a priori as function of time, since it may take several values for $t \in\left(\left(t_{i}^{\mu, \text { en }}, t_{i}^{\mu, \text { ex }}\right)\right)$ if $t_{i}^{\mu, \text { en }}>t_{i}^{\mu, \text { ex }}$ (where $((a, b))$ stands for $(a, b)$ if $a \leq b$ and $(b, a)$ otherwise). We will see that this last case cannot occur, i.e. (5.20) holds (and clearly also holds by continuity with a strict inequality for $i \in I_{\mathrm{ba}}$ ), and is satisfied with equality iff $\nu_{i}^{\mu, 1}=0$.

Note first that by (A2)-(A3) and the strong regularity property, for $\left\|\mu-\mu_{0}\right\|$ small enough, (4.20)-(4.21) are satisfied on each arc. Suppose first that $t_{i}^{\mu \text {,ex }}=t_{i}^{\mu \text {,en }}$. Then $\left(u, y, \eta_{1}, p_{1}\right)$ is defined as function of time without ambiguity in the neighborhood of $t_{i}^{\mu, \text { en }}$ (the algebraic variables are given by the dynamics on interior arcs). By (4.32)-(4.35), there is a jump of $p_{1}$ at entry time and no jump at exit time, and thus $\left(y, p_{1}\right)\left(t_{i}^{\mu, \text { en+ }}\right)=$ $\left(y, p_{1}\right)\left(t_{i}^{\mu, \mathrm{ex}-}\right)=\left(y, p_{1}\right)\left(t_{i}^{\mu, \mathrm{ex}+}\right)$. By definition of the problem (4.40), we have

$$
\left(g^{\mu}\right)^{(1)}\left(u\left(t_{i}^{\mu, \mathrm{en}-}\right), y\left(t_{i}^{\mu, \mathrm{en}}\right)\right)=\left(g^{\mu}\right)^{(1)}\left(u\left(t_{i}^{\mu, \mathrm{ex}+}\right), y\left(t_{i}^{\mu, \mathrm{ex}}\right)\right)=0,
$$

and hence, since $t_{i}^{\mu, \mathrm{ex}}=t_{i}^{\mu, \mathrm{en}},(4.21)$ implies that $u$ is continuous at time $t_{i}^{\mu, \mathrm{en}}$. We deduce that:

$$
0=\left[H_{u}^{\mu}\left(u\left(t_{i}^{\mu, \mathrm{en}}\right), y\left(t_{i}^{\mu, \mathrm{en}}\right), p_{1}\left(t_{i}^{\mu, \mathrm{en}}\right)\right)\right]=-\nu_{i}^{\mu, 1}\left(g^{\mu}\right)_{u}^{(1)}\left(u\left(t_{i}^{\mu, \mathrm{en}}\right), y\left(t_{i}^{\mu, \mathrm{en}}\right)\right) .
$$

Since $\left(g^{\mu}\right)_{u}^{(1)}\left(u\left(t_{i}^{\mu, \text { en }}\right), y\left(t_{i}^{\mu, \text { en }}\right)\right) \neq 0$ by (4.21), it follows that $\nu_{i}^{\mu, 1}=0$. This proves the " $\Rightarrow$ " implication in (5.21).

Suppose now that $t_{i}^{\mu, \text { ex }} \neq t_{i}^{\mu, \text { en }}$. In order to avoid any confusion, denote the solution of (4.32)-(4.35) for $\theta=\theta^{\mu}$ by $\left(u^{-}, y^{-}, p_{1}^{-}, \eta_{1}^{-}\right)$on the boundary $\operatorname{arc}\left(\left(t_{i}^{\mu, \text { en }}, t_{i}^{\mu, \mathrm{ex}}\right)\right)$, and by $\left(u^{+}, y^{+}, p_{1}^{+}, \eta_{1}^{+}\right)$on the succeeding interior $\operatorname{arc}\left(t_{i}^{\mu, \text { ex }}, t_{i+1}^{\mu, \text { en }}\right)$. Note that the limits of these functions and of their time derivative at endpoints of the interval where they are defined do exist, and are continuous w.r.t. $\mu$ (this follows from the implicit function theorem applied by (4.20)-(4.21) on each arc of the trajectory). This holds in particular for $\dot{u}^{\mu}$. Here the jump has the following signification, for instance $\left[u\left(t_{i}^{\mu, \mathrm{ex}}\right)\right]:=u^{+}\left(t_{i}^{\mu, \mathrm{ex}}\right)-u^{-}\left(t_{i}^{\mu, \mathrm{ex}}\right)$.

Since (4.20)-(4.21) are satisfied, we can show using the same local arguments as in Lemma 4.6 that

$$
\left(u^{+}, y^{+}, p_{1}^{+}, \eta_{1}^{+}\right)\left(t_{i}^{\mu, \mathrm{ex}}\right)=\left(u^{-}, y^{-}, p_{1}^{-}, \eta_{1}^{-}\right)\left(t_{i}^{\mu, \mathrm{ex}}\right),
$$


and we denote this common value by $\left(u\left(t_{i}^{\mu, \text { ex }}\right), y\left(t_{i}^{\mu \text {,ex }}\right), p_{1}\left(t_{i}^{\mu \text {,ex }}\right), \eta_{1}\left(t_{i}^{\mu, \text { ex }}\right)\right)$. By (A6), there exists by continuity a constant $c>0$ such that, for $\mu$ close enough to $\mu_{0}$,

$$
\lim _{t \rightarrow t_{i}^{\mu, \text { ex }}+} \frac{\mathrm{d}}{\mathrm{d} t}\left(g^{\mu}\right)^{(1)}\left(u^{+}(t), y^{+}(t)\right)<-c .
$$

On the other hand, we have on the boundary $\operatorname{arc}\left(\left(t_{i}^{\mu, \text { en }}, t_{i}^{\mu, \text { ex }}\right)\right)$ :

$$
\lim _{t \rightarrow t_{i}^{\mu, \mathrm{ex}}} \frac{\mathrm{d}}{\mathrm{d} t}\left(g^{\mu}\right)^{(1)}\left(u^{-}(t), y^{-}(t)\right)=0 .
$$

Since $\frac{\mathrm{d}}{\mathrm{d} t}\left(g^{\mu}\right)^{(1)}\left(u^{ \pm}(t), y^{ \pm}(t)\right)=\left(g^{\mu}\right)^{(2)}\left(\dot{u}^{ \pm}, u^{ \pm}, y^{ \pm}\right)$with $\left(g^{\mu}\right)^{(2)}$ given by (1.5), the jump of $\dot{u}$ at $t_{i}^{\mu, \text { ex }}$ satisfies

$$
\left(g^{\mu}\right)_{u}^{(1)}\left(u\left(t_{i}^{\mu, \mathrm{ex}}\right), y\left(t_{i}^{\mu, \mathrm{ex}}\right)\right)\left[\dot{u}\left(t_{i}^{\mu, \mathrm{ex}}\right)\right]=\left[\left.\frac{\mathrm{d}}{\mathrm{d} t}\left(g^{\mu}\right)^{(1)}(u(t), y(t))\right|_{t=t_{i}^{\mu, \mathrm{ex}}}\right]<-c,
$$

and hence, $\dot{u}^{-}\left(t_{i}^{\mu, \text { ex }}\right) \neq \dot{u}^{+}\left(t_{i}^{\mu, \text { ex }}\right)$. By time-derivation of (4.3) on the boundary arc $\left(\left(t_{i}^{\mu, \text { en }}, t_{i}^{\mu, \text { ex }}\right)\right)$ of nonzero length and on the interior $\operatorname{arc}\left(t_{i}^{\mu, \text { ex }}, t_{i+1}^{\mu, \text { en }}\right)$, we obtain (omitting $\left.\operatorname{arguments}\left(u^{ \pm}(t), y^{ \pm}(t), p_{1}^{ \pm}(t), \eta_{1}^{ \pm}(t)\right)\right)$ :

$$
\tilde{H}_{u u}^{\mu} \dot{u}^{ \pm}+\tilde{H}_{y u}^{\mu} f^{\mu}-\tilde{H}_{y}^{\mu} f_{u}^{\mu}+\left(g^{\mu}\right)_{u}^{(1)} \dot{\eta}_{1}^{ \pm}=0 .
$$

Hence, taking the jump at time $t_{i}^{\mu, \text { ex }}$ gives, since $\left(u, y, p_{1}, \eta_{1}\right)$ is continuous at $t_{i}^{\mu, \text { ex }}$ by (5.24):

$$
\tilde{H}_{u u}^{\mu}\left(u, y, p_{1}, \eta_{1}\right)\left(t_{i}^{\mu, \mathrm{ex}}\right)\left[\dot{u}\left(t_{i}^{\mu, \mathrm{ex}}\right)\right]+\left(g^{\mu}\right)_{u}^{(1)}(u, y)\left(t_{i}^{\mu, \mathrm{ex}}\right)\left[\dot{\eta}_{1}\left(t_{i}^{\mu, \mathrm{ex}}\right)\right]=0 .
$$

Since $\dot{\eta}_{1}^{+}\left(t_{i}^{\mu, \text { ex }}\right)=0$, by (5.27) and (4.20)-(4.21) there exists by continuity a constant $C>0$ such that, for $\left\|\mu-\mu_{0}\right\|$ small enough,

$$
\dot{\eta}_{1}^{-}\left(t_{i}^{\mu, \mathrm{ex}}\right)=-\left[\dot{\eta}_{1}\left(t_{i}^{\mu, \mathrm{ex}}\right)\right]=\frac{\tilde{H}_{u u}^{\mu}\left(u, y, p_{1}, \eta_{1}\right)\left(t_{i}^{\mu, \mathrm{ex}}\right)}{\left(\left(g^{\mu}\right)_{u}^{(1)}(u, y)\left(t_{i}^{\mu, \mathrm{ex}}\right)\right)^{2}}\left(g^{\mu}\right)_{u}^{(1)}(u, y)\left(t_{i}^{\mu, \mathrm{ex}}\right)\left[\dot{u}\left(t_{i}^{\mu, \mathrm{ex}}\right)\right]<-C .
$$

By (5.28) and time derivation of (4.4), we see that $\dot{\eta}_{1}^{-}(t)$ is given by a Lipschitz continuous function of time on $\left(\left(t_{i}^{\mu, \text { en }}, t_{i}^{\mu \text {,ex }}\right)\right)$, uniformly w.r.t. $\mu$, so there exists $m>0$ independent of $\mu$, such that

$$
\dot{\eta}_{1}^{-}(t) \leq-C+m\left|t_{i}^{\mu, \mathrm{ex}}-t_{i}^{\mu, \mathrm{en}}\right|, \quad t \in\left(\left(t_{i}^{\mu, \mathrm{en}}, t_{i}^{\mu, \mathrm{ex}}\right)\right) .
$$

In view of (5.23), this implies that $\dot{\eta}_{1}^{-}$is negative on $\left(\left(t_{i}^{\mu, \text { en }}, t_{i}^{\mu, \text { ex }}\right)\right)$ for sufficiently small $\left\|\mu-\mu_{0}\right\|$, and consequently, $\eta_{1}^{-}\left(t_{i}^{\mu, \text { en }}\right)=\eta_{1}^{-}\left(t_{i}^{\mu \text {,en }}\right)-\eta_{1}^{-}\left(t_{i}^{\mu, \text { ex }}\right)$ is nonzero and has the sign of $t_{i}^{\mu, \text { ex }}-t_{i}^{\mu, \text { en }}$. By similar arguments to Lemma 4.6, we can show that $\eta_{1}^{-}\left(t_{i}^{\mu, \text { en }}\right)=\nu_{i}^{\mu, 1}$, and since $\nu_{i}^{\mu, 1} \geq 0$ by definition of the problem (4.40), it follows that $t_{i}^{\mu, \mathrm{ex}}>t_{i}^{\mu, \mathrm{en}}$ necessarily holds whenever $t_{i}^{\mu, \mathrm{en}} \neq t_{i}^{\mu, \mathrm{ex}}$, which proves (5.20). In addition, (5.30) implies that $\nu_{i}^{\mu, 1}=\eta_{1}\left(t_{i}^{\mu, \text { en }+}\right)>0$ for $\mu$ close enough to $\mu_{0}$, which show by contraposition the " $\Leftarrow$ " implication in (5.21). Finally, relation (5.22) follows from (5.29) and (5.23), which completes the proof.

Lemma 5.3. Under assumptions (A2)-(A6) and (3.8), the solution $\left(u^{\mu}, y^{\mu}, p_{1}^{\mu}, \eta_{1}^{\mu}\right)$ of (4.32)-(4.35) for $\theta=\theta^{\mu}$, where $\theta^{\mu}$ is solution of (4.40), is, for $\left\|\mu-\mu_{0}\right\|$ small enough, such that $\left(u^{\mu}, y^{\mu}\right)$ is a stationary point of $\left(\mathcal{P}^{\mu}\right)$, with classical multipliers $\left(p^{\mu}, \eta^{\mu}\right)$ given by (4.16), and the mapping $\mu \mapsto\left(u^{\mu}, y^{\mu}, p^{\mu}, \eta^{\mu}\right) \in C^{0}[0, T] \times C^{0}\left([0, T] ; \mathbb{R}^{n}\right) \times$ $C^{0}\left([0, T] ; \mathbb{R}^{n *}\right) \times C^{0}[0, T]$ is Lipschitz continuous on a neighborhood of $\mu_{0}$.

Proof. By Lemma 5.2, we see that $\left(u^{\mu}, y^{\mu}, p_{1}^{\mu}, \eta_{1}^{\mu}\right)$ is well-defined over $[0, T]$, and by definition of the problem (4.40), satisfies the alternative formulation (4.1)-(4.11). By (A2)-(A3), (4.20)-(4.21) hold for $\left\|\mu-\mu_{0}\right\|$ small enough, so Lemma 4.6 implies that the additional condition (4.14) is satisfied, and that $u^{\mu}$ is continuous 
on $[0, T]$, as well as $\eta^{\mu}$ and $p^{\mu}$ given by (4.16). In view of Remark 4.3, in order to show that $\left(u^{\mu}, y^{\mu}\right)$ is a stationary point of $\left(\mathcal{P}^{\mu}\right)$ it remains to show that the additional conditions (4.12), (4.13) and (4.15) are satisfied. By (4.20)-(4.21), the implicit function theorem applied on each arc shows that $\dot{u}^{\mu}\left(t_{i}^{\mu, \text { en-}}\right)$ and $\dot{u}^{\mu}\left(t_{i}^{\mu, \text { ex }+}\right)$ are continuous w.r.t. $\mu$, for all $i=1, \ldots, N$, as well as $\dot{\eta}_{1}^{\mu}\left(t_{i}^{\mu, \text { en }+}\right)$ and $\dot{\eta}_{1}^{\mu}\left(t_{i}^{\mu, \text { ex }}{ }^{-}\right)$for $i \in I_{\text {ba }}$. So let $\left\|\mu-\mu_{0}\right\|$ be so small that, by (1.25)-(1.27) and (1.6),

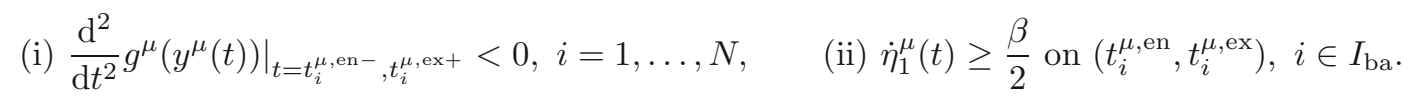

Let $i \in N_{\mathrm{to}}$. If $\nu_{i}^{\mu, 1}=0$, then by Lemma $5.2, t_{i}^{\mu, \mathrm{en}}=t_{i}^{\mu, \mathrm{ex}}, u^{\mu}$ and its time derivative are continuous at $t_{i}^{\mu, \text { en }}$, and $\left(g^{\mu}\right)^{(1)}\left(u^{\mu}\left(t_{i}^{\mu, \text { en }}\right), y^{\mu}\left(t_{i}^{\mu, \text { en }}\right)\right)=0$. By $(\mathrm{A} 6)$ and standard continuity arguments, there exists $\varepsilon>0$ such that $g^{\mu}\left(y^{\mu}(\cdot)\right)$ attains its maximum over $\left(\bar{t}_{i}^{\text {en }}-\varepsilon, \bar{t}_{i}^{\text {en }}+\varepsilon\right)$ at the unique point $t_{i}^{\mu, \text { en }}$. Therefore if $g^{\mu}\left(y^{\mu}\left(t_{i}^{\mu, \text { en }}\right)\right)<0$, the state constraint is locally not active. If $g^{\mu}\left(y^{\mu}\left(t_{i}^{\mu, \text { en }}\right)\right)=0$, then $t_{i}^{\mu, \text { en }}$ is a touch point of the perturbed problem, and (4.15) holds by (5.21). If $\nu_{i}^{\mu, 1}>0$, then by Lemma $5.2, t_{i}^{\mu, \text { en }}<t_{i}^{\mu, \text { ex }}$ and we have a boundary arc. By (5.22), additional condition (4.13) holds on this boundary arc. If $i \in I_{\text {ba }}$, then (4.13) holds on the boundary $\operatorname{arc}\left(t_{i}^{\mu, \mathrm{en}}, t_{i}^{\mu, \mathrm{ex}}\right)$ by (5.31)(ii). Finally, (4.12) holds near the junction points by (5.31)(i), and outside a small neighborhood of contact points, we obtain $g^{\mu}\left(y^{\mu}\right)<0$ by a standard compactness argument. Hence $\left(u^{\mu}, y^{\mu}\right)$ is a stationary point, with classical multipliers $\left(p^{\mu}, \eta^{\mu}\right)$ given by (4.16).

Lipschitz continuity of the mapping $\mu \mapsto\left(u^{\mu}, y^{\mu}, p^{\mu}, \eta^{\mu}\right)$ follows from Lipschitz continuity of the mapping $\mu \mapsto$ $\theta^{\mu}$ by strong regularity (Lem. 5.1), Lipschitz continuity of $\left.(\theta, \mu) \mapsto(u, y, p, \eta)\right|_{k}$, where $\left.(u, y, p, \eta)\right|_{k}$ denotes the restriction of the solution of (4.32)-(4.35) and (4.16) to "arc" $k$ (possibly a singleton), for all $k=1, \ldots, 2 N+1$, and continuity of $u^{\mu}, \dot{y}^{\mu}, p^{\mu}$ and $\eta^{\mu}$ on $[0, T]$.

Thanks to Theorem 2.1, we can show that $\left(u^{\mu}, y^{\mu}\right)$ is the locally unique stationary point of $\left(\mathcal{P}^{\mu}\right)$.

Lemma 5.4. Under assumptions (A2)-(A6) and (3.8), there exist a $L^{\infty}$ neighborhood $V_{u}$ of $\bar{u}$ and a neighborhood $V_{\mu}$ of $\mu_{0}$, such that for all $\mu \in V_{\mu},\left(u^{\mu}, y^{\mu}\right)$ is the locally unique stationary point of $\left(\mathcal{P}^{\mu}\right)$ with $u \in V_{u}$.

Proof. Let $(u, y)$ be a stationary point of $\left(\mathcal{P}^{\mu}\right)$ with $(u, \mu)$ in the neighborhood of $\left(\bar{u}, \mu_{0}\right)$. By Theorem 2.1, $(u, y)$ satisfies $(\mathrm{S} 1)-(\mathrm{S} 3)$, and therefore has finitely many junction times, so it makes sense to speak of the finitedimensional vector of "shooting parameters" $\theta$ (initial costate, jump parameters at entry times, and junction times) such that $(u, y)$ is solution of the alternative formulation (Def. 4.1). Now construct its augmented set of shooting parameters $\hat{\theta}$ as follows. For all $i \in I_{\text {to }}$, if the state constraint is not active on $\Omega_{i}^{\delta}$, add to the set of shooting parameters $\theta$ the (unique by (A6)) time in $\Omega_{i}^{\delta}$ where $g^{\mu}(y)$ attains its maximum over $\Omega_{i}^{\delta}$, duplicate all such times as well as touch points, add a zero jump parameter for each of them, and obtain then a $\hat{\theta} \in \Theta$ such that $\hat{\theta}$ is solution of $(4.40)$, and $(u, y)$ is the trajectory associated with $\hat{\theta}$.

Let us show that this augmented set of shooting parameters $\hat{\theta}$ is arbitrarily close to $\theta_{0}$ when $\left\|\mu-\mu_{0}\right\|$ and $\|u-\bar{u}\|_{\infty}$ are small enough. Indeed, the convergence of the initial costate is a consequence of Remark 2.4. For $i \in I_{\mathrm{ba}}$, since we know by Theorem 2.1 that $\Omega_{i}^{\delta} \cap I\left(g^{\mu}(y)\right)$ is an interval $\left[\tau_{\mathrm{en}, i}^{\mu}, \tau_{\mathrm{ex}, i}^{\mu}\right]$, letting $\delta \rightarrow 0$, we obtain that $\bar{t}_{i}^{\text {en }} \leq \liminf _{\mu \rightarrow \mu_{0}} \tau_{\mathrm{en}, i}^{\mu}$ and $\bar{t}_{i}^{\text {ex }} \geq \lim \sup _{\mu \rightarrow \mu_{0}} \tau_{\mathrm{ex}, i}^{\mu}$. The converse inequalities $\bar{t}_{i}^{\text {en }} \geq \lim _{\sup _{\mu \rightarrow \mu_{0}}} \tau_{\mathrm{en}, i}^{\mu}$ and $\bar{t}_{i}^{\mathrm{ex}} \leq \liminf \operatorname{in}_{\mu \rightarrow \mu_{0}} \tau_{\mathrm{ex}, i}^{\mu}$ are obtained as follows. Assume e.g. by contradiction that $\bar{t}_{i}^{\mathrm{en}}<\lim \sup _{\mu \rightarrow \mu_{0}} \tau_{\mathrm{en}, i}^{\mu}$. Then there exist $\delta>0$, a stable extension $\left(\mathcal{P}^{\mu}\right)$, a sequence $\mu_{n} \rightarrow \mu_{0}$, and a stationary point $\left(u_{n}, y_{n}\right)$ of $\left(\mathcal{P}^{\mu_{n}}\right)$, with multipliers $\left(p_{n}, \eta_{n}\right)$, such that $u_{n} \rightarrow \bar{u}$ in $L^{\infty}$ and $\tau_{\text {en }, i}^{\mu_{n}} \geq \bar{t}_{i}^{\text {en }}+\delta$ for all $n$. Let $\varphi$ be a $C^{\infty}$ function with support in $\left[\bar{t}_{i}^{\mathrm{en}}, \bar{t}_{i}^{\mathrm{en}}+\delta\right]$ and positive on $\left(\bar{t}_{i}^{\mathrm{en}}, \bar{t}_{i}^{\mathrm{en}}+\delta\right)$. Then $\int_{0}^{T} \varphi(t) \mathrm{d} \eta_{n}(t)=0$, for all $n$. But by $(\mathrm{A} 5), \int_{0}^{T} \varphi(t) \mathrm{d} \bar{\eta}(t)>0$, which contradicts the second assertion in Lemma 2.3. This achieves to show the convergence of entry/exit points for $i \in I_{\mathrm{ba}}$. Letting $\delta \rightarrow 0$ in (S3), we obtain similarly the convergence of touch points and entry/exit points of boundary arcs to the common value $\bar{t}_{i}^{\text {en }}$, for all $i \in I_{\text {to }}$. The convergence of nonactive local isolated maxima of $g^{\mu}(y)$ in $\Omega_{i}^{\delta}$ when $i \in I_{\text {to }}$, is obtained by classical arguments, since (1.26) holds and locally on $\Omega_{i}^{\delta}$, the second-order derivative (1.6) is continuous on interior arcs since $u$ and $\dot{u}$ are (indeed, for $\|u-\bar{u}\|_{\infty}$ and $\left\|\mu-\mu_{0}\right\|$ small enough, $H_{u u}^{\mu}(u, y, p) \geq \alpha / 2>0$ by (A2) and Rem. 2.4), so that $g^{\mu}(y)$ belongs to a $C^{2}$ neighborhood 
of $g^{\mu_{0}}(\bar{y})$. Finally, the convergence of jump parameters at entry times follows from assertion (4) in Lemma 2.3, since $\eta^{1}$ and $\eta_{1}$ are related by (4.18), and $\eta_{1}$ satisfies (4.5) and (4.14).

Hence if $(\mu, u)$ is close enough to $\left(\mu_{0}, \bar{u}\right)$, the augmented set of shooting parameters $\hat{\theta}$ belongs to the neighborhood $V_{\theta}$ of $\theta_{0}$, on which (4.40) has a unique solution $\theta^{\mu}$ by Lemma 5.1, and $(u, y)$ is the (unique) trajectory associated with $\hat{\theta}$. Consequently, $\hat{\theta}=\theta^{\mu}$ and $(u, y)=\left(u^{\mu}, y^{\mu}\right)$ is the unique stationary point of $\left(\mathcal{P}^{\mu}\right)$ with $(u, \mu)$ in the neighborhood of $\left(\bar{u}, \mu_{0}\right)$.

Now we can prove the main result. Under assumptions (A2)-(A6) and point (ii) of Theorem 3.3, for $\mu$ in the neighborhood of $\mu_{0}$ and $v \in L^{2}$, denote by $z_{v}^{\mu}$ the unique solution in $\mathcal{Z}$ of the linearized state equation

$$
\dot{z}_{v}^{\mu}=f_{u}^{\mu}\left(u^{\mu}, y^{\mu}\right) v+f_{y}^{\mu}\left(u^{\mu}, y^{\mu}\right) z_{v}^{\mu} \quad \text { a.e. on }[0, T], \quad z_{v}^{\mu}(0)=0
$$

and by $Q^{\mu}$ the quadratic form over $L^{2}$ defined by

$$
Q^{\mu}(v)=\mathcal{J}^{\mu}\left(v, z_{v}^{\mu}\right)
$$

where $\mathcal{J}^{\mu}$ is defined by $(3.1)$ for $\left(\mathcal{P}^{\mu}\right)$ and its stationary point and multipliers $\left(u^{\mu}, y^{\mu}, p^{\mu}, \eta^{\mu}\right)$.

Proof of Theorem 3.3. By Lemmas 5.1-5.4, to achieve the proof of (ii) $\Rightarrow$ (i), it remains to show that $u^{\mu}$ satisfies the uniform quadratic growth condition. The arguments used are similar to those in the proof of [6], Theorem 4.3. We argue by contradiction. Assume that the uniform quadratic growth does not hold. Then there exist a sequence $\mu_{n}$ converging to $\mu_{0}$ and a sequence $u_{n} \rightarrow \bar{u}$ in $L^{\infty}$ such that for all $n, G^{\mu_{n}}\left(u_{n}\right) \in K$ and

$$
J^{\mu_{n}}\left(u_{n}\right) \leq J^{\mu_{n}}\left(u^{\mu_{n}}\right)+o\left(\left\|u_{n}-u^{\mu_{n}}\right\|_{2}^{2}\right)
$$

Introducing the Lagrangian of (1.11) defined by $L^{\mu}(u, \eta):=J^{\mu}(u)+\left\langle\eta, G^{\mu}(u)\right\rangle$, with $\langle\cdot, \cdot\rangle$ the duality product in $\mathcal{M}[0, T] \times C^{0}[0, T]$ defined by $\langle\eta, x\rangle=\int_{0}^{T} x(t) \mathrm{d} \eta(t)$, we obtain that

$$
L^{\mu_{n}}\left(u_{n}, \eta^{\mu_{n}}\right) \leq L^{\mu_{n}}\left(u^{\mu_{n}}, \eta^{\mu_{n}}\right)+o\left(\left\|u_{n}-u^{\mu_{n}}\right\|_{2}^{2}\right)
$$

Set $\varepsilon_{n}:=\left\|u_{n}-u^{\mu_{n}}\right\|_{2} \rightarrow 0$ and $v_{n}:=\left(u_{n}-u^{\mu_{n}}\right) / \varepsilon_{n}$. A second-order expansion of the Lagrangian shows that

$$
L^{\mu_{n}}\left(u_{n}, \eta^{\mu_{n}}\right)=L^{\mu_{n}}\left(u^{\mu_{n}}, \eta^{\mu_{n}}\right)+\varepsilon_{n}^{2} Q^{\mu_{n}}\left(v_{n}\right)+o\left(\varepsilon_{n}^{2}\right),
$$

where $Q^{\mu_{n}}$ is defined by (5.33). It follows then that $Q^{\mu_{n}}\left(v_{n}\right) \leq o(1)$. Since $\left(v_{n}\right)$ is bounded in $\mathcal{V}=L^{2}$, we may assume that it converges weakly to some $\bar{v} \in L^{2}$. In view of the compact inclusion of $H^{1}(0, T)$ in $C^{0}[0, T]$, the associated linearized state $z_{n}:=z_{v_{n}}^{\mu_{n}}$ defined by $(5.32)$ converges uniformly to $\bar{z}:=z_{\bar{v}}^{\mu_{0}}$. We may write that

$$
Q^{\mu_{n}}\left(v_{n}\right)=Q^{\mu_{0}}\left(v_{n}\right)+Q^{\mu_{n}}\left(v_{n}\right)-Q^{\mu_{0}}\left(v_{n}\right)
$$

and using that $\left\|v_{n}\right\|_{2}$ is bounded it is not difficult to check that $Q^{\mu_{n}}\left(v_{n}\right)-Q^{\mu_{0}}\left(v_{n}\right) \rightarrow 0$. Therefore by weak lower-semicontinuity of the Legendre form $Q=Q^{\mu_{0}}$ by (1.20), we obtain that

$$
\mathcal{J}(\bar{v}, \bar{z})=Q(\bar{v}) \leq \liminf _{n \rightarrow+\infty} Q\left(v_{n}\right) \leq \limsup _{n \rightarrow+\infty} Q\left(v_{n}\right) \leq 0
$$

Moreover, $\bar{v}$ and $\bar{z}$ satisfy the constraint (3.3). Indeed, since $G^{\mu_{n}}\left(u_{n}\right) \in K$, we have that $g_{y}^{\mu_{n}}\left(y^{\mu_{n}}\right) z_{n}+r_{n} \leq 0$ on $I\left(g^{\mu_{n}}\left(y^{\mu_{n}}\right)\right)$, where $r_{n}$ satisfies $\left\|r_{n}\right\|_{\infty}=\mathcal{O}\left(\varepsilon_{n}\right)$. Since $\frac{\mathrm{d}}{\mathrm{d} t} g_{y}^{\mu_{n}}\left(y^{\mu_{n}}(t)\right) z_{n}(t)=\left(g_{u}^{\mu_{n}}\right)^{(1)}\left(u^{\mu_{n}}, y^{\mu_{n}}\right) v_{n}+$ $\left(g_{y}^{\mu_{n}}\right)^{(1)}\left(u^{\mu_{n}}, y^{\mu_{n}}\right) z_{n}$, it follows from Cauchy-Schwarz inequality that the functions (of time) $g_{y}^{\mu_{n}}\left(y^{\mu_{n}}\right) z_{n}$ are 
uniformly Hölder continuous. Therefore, there exists a constant $C>0$ such that, for all large enough $n$, using Lemma 5.1,

$$
\sup _{t \in \cup_{i=1}^{N}\left[\bar{t}_{i}^{\text {en }}, \bar{t}_{i}^{\text {ex }}\right]} g_{y}^{\mu_{n}}\left(y^{\mu_{n}}(t)\right) z_{n}(t) \leq \mathcal{O}\left(\varepsilon_{n}\right)+C \sqrt{\max _{i=1, \ldots, N}\left\{\left|t_{i}^{\mu_{n}, \text { en }}-\bar{t}_{i}^{\text {en }}\right|,\left|t_{i}^{\mu_{n}, \text { ex }}-\bar{t}_{i}^{\text {ex }}\right|\right\}}=o(1) .
$$

Since $g_{y}^{\mu_{n}}\left(y^{\mu_{n}}\right) z_{n} \rightarrow g_{y}(\bar{y}) \bar{z}$ uniformly, it follows that $g_{y}(\bar{y}) \bar{z} \leq 0$ on $\cup_{i=1}^{N}\left[\bar{t}_{i}^{\mathrm{en}}, \bar{t}_{i}^{\mathrm{ex}}\right]$. In addition, by (5.34), we have that $\left\langle\eta^{\mu_{n}}, g_{y}^{\mu_{n}}\left(y^{\mu_{n}}\right) z_{n}\right\rangle=-D J^{\mu_{n}}\left(u^{\mu_{n}}\right) v_{n} \geq \mathcal{O}\left(\varepsilon_{n}\right)$. Therefore, $\left\langle\bar{\eta}, g_{y}(\bar{y}) \bar{z}\right\rangle \geq 0$, which implies finally by (A5) that $g_{y}(\bar{y}) \bar{z}=0$ on $\cup_{i=1}^{N}\left[\bar{t}_{i}^{\mathrm{en}}, \bar{t}_{i}^{\mathrm{ex}}\right]$, i.e. (3.3) holds. Thus (3.8) and (5.35) imply that $\bar{v}=0$. But then $Q\left(v_{n}\right) \rightarrow Q(\bar{v})$, and hence, by the property of Legendre forms, $v_{n} \rightarrow \bar{v}$ strongly, contradicting that $\left\|v_{n}\right\|_{2}=1$ for all $n$.

To prove the converse implication, we construct a perturbation of the constraint $g^{\mu}$, so that (nonessential) touch points becomes inactive on the perturbed problem $\left(\mathcal{P}^{\mu}\right)$, and $(\bar{u}, \bar{y})$ is a stationary point of $\left(\mathcal{P}^{\mu}\right)$. This is where we need nonautonomous perturbations. Let $\varphi$ be a $C^{\infty}$ function with support in $[-1,1]$ and positive on $(-1,1)$. Set $\mu_{0}=0$ and $g^{\mu}(y):=g(y)-\sum_{\tau \in \overline{\mathcal{T}}_{\mathrm{to}}} \mu^{5} \varphi\left(\left(y_{n}-\tau\right) / \mu\right)$ for $\mu \neq 0$ (recall that we assume $(1.4)$ ). Then $\left(\ell, \phi, f, g^{\mu}, y_{0}\right)$ is a stable extension of $(\mathcal{P}),(\bar{u}, \bar{y})$ is a stationary point of $\left(\mathcal{P}^{\mu}\right)$ for all $|\mu|$ small enough, and $g^{\mu}(\bar{y}(\tau))<0$ for all nonessential touch point $\tau$. By the definition of the uniform growth condition, $(\bar{u}, \bar{y})$ is a local solution of $\left(\mathcal{P}^{\mu}\right)$ satisfying (3.9), so it follows from Theorem 3.1(ii) that the strong second-order sufficient condition (3.8) holds.

We end this section by the proof of Lemma 3.4.

Proof of Lemma 3.4. Denote by $Q^{\mu}$ the quadratic form (5.33) and $\tilde{C}^{\mu}$ the set of $v \in \mathcal{V}$ satisfying the constraints $(3.2)-(3.3)$ for $\left(\mathcal{P}^{\mu}\right)$ and its stationary point $\left(u^{\mu}, y^{\mu}\right)$, i.e. such that

$$
g_{y}^{\mu}\left(y^{\mu}\right) z_{v}^{\mu}=0 \quad \text { on }\left[t_{i}^{\mu, \mathrm{en}}, t_{i}^{\mu, \mathrm{ex}}\right], \quad \text { for all } i=1, \ldots, N
$$

Let us show that there exists $\alpha^{\prime}>0$ such that for all $\mu$ close enough to $\mu_{0}$ and all $v \in \tilde{C}^{\mu}\left(u^{\mu}\right)$, we have $Q^{\mu}(v) \geq \alpha^{\prime}\|v\|_{2}^{2}$, which will give the result.

We argue by contradiction, as in the proof of the uniform growth condition in Theorem 3.3. Assume this is not the case. Then there exist sequences $\left(\mu_{n}\right)_{n \in \mathbb{N}^{*}}$ and $\left(v_{n}\right)_{n \in \mathbb{N}^{*}}$, such that $\mu_{n} \rightarrow \mu_{0}, v_{n} \in \tilde{C}^{\mu_{n}}$ for all $n$, and

$$
Q^{\mu_{n}}\left(v_{n}\right) \leq o(1)\left\|v_{n}\right\|_{2}^{2}
$$

Since $\tilde{C}^{\mu_{n}}$ is a cone (in fact, here, a linear subspace of $\mathcal{V}$ ), and $Q^{\mu_{n}}$ is a quadratic form, assume w.l.o.g. that $\left\|v_{n}\right\|_{2}=1$, and taking a subsequence if necessary, that the sequence $\left(v_{n}\right)$ converges weakly to some $\bar{v} \in \mathcal{V}$. Then the associated state $z_{n}:=z_{v_{n}}^{\mu_{n}}$ given by (5.32) is weakly convergent to $\bar{z}:=z_{\bar{v}}^{\mu_{0}}$ in $H^{1}$, and hence $z_{n} \rightarrow \bar{z}$ uniformly. By the same argument as in the proof of Theorem 3.3 (see (5.36)), since $v_{n} \in \tilde{C}^{\mu_{n}}$, we deduce that $\sup _{t \in \cup_{i=1}^{N}\left[\bar{t}_{i}^{\text {en }}, \bar{t}_{i}^{\text {ex }}\right]}\left|g_{y}^{\mu_{n}}\left(y^{\mu_{n}}(t)\right) z_{n}(t)\right| \leq C \sqrt{\max _{i=1, \ldots, N}\left\{\left|t_{i}^{\mu_{n}, \text { en }}-\bar{t}_{i}^{\text {en }}\right|,\left|t_{i}^{\mu_{n}, \text { ex }}-\bar{t}_{i}^{\text {ex }}\right|\right\}}=o(1)$. It follows then that $\bar{v} \in \tilde{C}^{\mu_{0}}$. But (5.38) implies that $Q^{\mu_{0}}(\bar{v}) \leq 0$, therefore $\bar{v}=0$ by (3.8), and then $Q^{\mu_{0}}\left(v_{n}\right) \rightarrow Q^{\mu_{0}}(\bar{v})$. Since $Q^{\mu_{0}}$ is a Legendre form, it follows that $v_{n} \rightarrow \bar{v}$ strongly, contradicting that $\left\|v_{n}\right\|_{2}=1$ for all $n$. This achieves the proof.

\section{Sensitivity ANALYSiS}

Under assumptions (A2)-(A6) and point (i) or (ii) of Theorem 3.3, we investigate in this section directional differentiability of solutions. Given a stable extension $\left(\mathcal{P}^{\mu}\right)$, by Lemma 5.1, strong regularity holds, and the mapping $\Xi: V_{\delta} \rightarrow V_{\theta}^{\prime}, \delta \mapsto \theta(\delta)$ solution of (5.1) is given by $\Xi(\delta)=\theta_{0}+\omega(\delta)$, where $\omega(\delta)$ is the solution of (5.5). It is easy to see that the mapping $\delta \mapsto \omega(\delta)$ is positively homogeneous of degree one, and it follows 
then from (5.4) that the mapping $\mu \mapsto \theta^{\mu}$ is Fréchet directionally differentiable. The directional derivatives in direction $d \in M$ are obtained by substituting into (5.5) $\delta$ by $-D_{\mu} F\left(\theta_{0}, \mu_{0}\right) d$. Therefore,

$$
\theta^{\mu_{0}+d}=\theta_{0}+\omega_{d}+o(\|d\|)
$$

where

is as follows. Denote by

$$
\omega_{d}=\left(\pi_{d, 0}^{*}, \gamma_{d}^{1}, \sigma_{d}^{\mathrm{en}}, \sigma_{d}^{\mathrm{ex}}\right) \in \mathbb{R}^{n} \times \mathbb{R}^{N} \times \mathbb{R}^{N} \times \mathbb{R}^{N}
$$

$$
\left(v_{d}, z_{d}, \pi_{1, d}, \zeta_{1, d}, \lambda_{d}^{1}\right)
$$

the (unique) optimal solution, costate and multipliers of the linear-quadratic problem below:

$$
\begin{aligned}
& \min _{(v, z) \in \mathcal{V} \times \mathcal{Z}} \frac{1}{2} \int_{0}^{T} D_{(u, y, \mu),(u, y, \mu)}^{2} \tilde{H}\left(\bar{u}, \bar{y}, \bar{p}_{1}, \bar{\eta}_{1}, \mu_{0}\right)((v, z, d),(v, z, d)) \mathrm{d} t \\
& +\frac{1}{2} D^{2} \hat{\phi}\left(\bar{y}(T), \mu_{0}\right)((z(T), d),(z(T), d)) \\
& +\frac{1}{2} \sum_{i \in I_{\mathrm{ba}}} \bar{\nu}_{i}^{1} D^{2} \hat{g}\left(\bar{y}\left(\bar{t}_{i}^{\mathrm{en}}\right), \mu_{0}\right)\left(\left(z\left(\bar{t}_{i}^{\mathrm{en}}\right), d\right),\left(z\left(\bar{t}_{i}^{\mathrm{en}}\right), d\right)\right) \\
& \text { subject to: } \\
& \dot{z}=D \hat{f}\left(\bar{u}, \bar{y}, \mu_{0}\right)(v, z, d) \quad \text { on }[0, T], \quad z(0)=D \hat{y}_{0}\left(\mu_{0}\right) d \\
& D \hat{g}^{(1)}\left(\bar{u}, \bar{y}, \mu_{0}\right)(v, z, d)=0 \quad \text { on } \overline{\mathcal{I}}_{b} \\
& D \hat{g}\left(\bar{y}\left(\bar{t}_{i}^{\mathrm{en}}\right), \mu_{0}\right)\left(z\left(\bar{t}_{i}^{\mathrm{en}}\right), d\right)=0, \quad i \in I_{\mathrm{ba}} \\
& D \hat{g}\left(\bar{y}\left(\bar{t}_{i}^{\mathrm{en}}\right), \mu_{0}\right)\left(z\left(\bar{t}_{i}^{\mathrm{en}}\right), d\right) \leq 0, \quad i \in I_{\mathrm{to}},
\end{aligned}
$$

with $\pi_{1, d}$ associated with the constraint (6.4), $\zeta_{1, d}$ with (6.5), and $\lambda_{d}^{1}$ with (6.6)-(6.7). Then we have

$$
\begin{aligned}
\pi_{d, 0} & =\pi_{1, d}(0) \\
\gamma_{d}^{1} & =\lambda_{d}^{1} \\
\sigma_{d, i}^{\mathrm{en}} & =-\frac{D \hat{g}^{(1)}\left(\bar{u}\left(\bar{t}_{i}^{\mathrm{en}}\right), \bar{y}\left(\bar{t}_{i}^{\mathrm{en}}\right), \mu_{0}\right)\left(v_{d}\left(\bar{t}_{i}^{\mathrm{en}-}\right), z_{d}\left(\bar{t}_{i}^{\mathrm{en}}\right), d\right)}{\left.\frac{\mathrm{d}}{\mathrm{d} t} g^{(1)}(\bar{u}, \bar{y})\right|_{t=\bar{t}_{i}^{\mathrm{en}}-}}, i=1, \ldots, N \\
\sigma_{d, i}^{\mathrm{ex}} & =-\frac{D \hat{g}^{(1)}\left(\bar{u}\left(\bar{t}_{i}^{\mathrm{ex}}\right), \bar{y}\left(\bar{t}_{i}^{\mathrm{ex}}\right), \mu_{0}\right)\left(v_{d}\left(\bar{t}_{i}^{\mathrm{ex}+}\right), z_{d}\left(\bar{t}_{i}^{\mathrm{ex}}\right), d\right)}{\left.\frac{\mathrm{d}}{\mathrm{d} t} g^{(1)}(\bar{u}, \bar{y})\right|_{t=\bar{t}_{i}^{e x+}}}, i=1, \ldots, N .
\end{aligned}
$$

Since the mapping $\mu \mapsto \theta^{\mu}$ is Fréchet directionally differentiable, and the solution $\left(u^{\mu}, y^{\mu}, p_{1}^{\mu}, \eta_{1}^{\mu}\right)$ of $(4.32)-$ (4.36) is, on each arc, a $C^{1}$ function of $\left(\theta^{\mu}, \mu\right)$, combining with the continuity of $u^{\mu}$ and of the classical multipliers $p^{\mu}$ and $\eta^{\mu}$ given by (4.16) (which follows from Lem. 5.3), we obtain the following result.

Theorem 6.1. Let $(\bar{u}, \bar{y})$ be a stationary point of $(\mathcal{P})$ satisfying (A2)-(A6). If either point (i) or (ii) of Theorem 3.3 is satisfied, then there exists a neighborhood $V_{\mu}$ of $\mu$, such that the mapping $\mu \mapsto\left(u^{\mu}, y^{\mu}, p^{\mu}, \eta^{\mu}\right)$ is Fréchet directionally differentiable in the space

$$
L^{r}(0, T) \times W^{1, r}\left(0, T ; \mathbb{R}^{n}\right) \times L^{r}\left(0, T ; \mathbb{R}^{n *}\right) \times L^{r}(0, T), \quad \text { for all } 1 \leq r<+\infty,
$$

and the derivatives of the state and control in direction $d$ are the optimal solution $\left(v_{d}, z_{d}\right)$ of linear-quadratic problem $\left(\mathcal{P}_{d}\right)$, while those of the costate $p^{\mu}$ and state constraint multiplier $\eta^{\mu}$ are obtained, respectively, a.e. by

$$
\begin{aligned}
& \pi_{d}(t)=\pi_{1, d}(t)+\zeta_{1, d}(t) g_{y}^{\mu_{0}}(\bar{y}(t))+\bar{\eta}_{1}(t) D \hat{g}_{y}\left(\bar{y}(t), \mu_{0}\right)\left(z_{d}(t), d\right) \\
& \zeta_{d}(t)=-\sum_{i=1}^{N} \gamma_{d, i}^{1} \mathbf{1}_{\left[0, \bar{t}_{i}^{\mathrm{en}}\right)}(t)-\zeta_{1, d}(t)
\end{aligned}
$$


In addition, all shooting parameters (initial costate, jump parameters and junction times) are Fréchet directionally differentiable w.r.t. $\mu$, and their directional derivative in direction d are given by (6.8)-(6.11).

Remark 6.2. We can show that an equivalent formulation of $\left(\mathcal{P}_{d}\right)$ is (see Rem. 4.10) to minimize

$$
\begin{gathered}
\int_{0}^{T} D_{(u, y, \mu),(u, y, \mu)}^{2} H\left(\bar{u}, \bar{y}, \bar{p}, \mu_{0}\right)((v, z, d),(v, z, d)) \mathrm{d} t+D^{2} \hat{\phi}\left(\bar{y}(T), \mu_{0}\right)((z(T), d),(z(T), d)) \\
+\int_{0}^{T} D^{2} \hat{g}\left(\bar{y}(t), \mu_{0}\right)((z(t), d),(z(t), d)) \mathrm{d} \bar{\eta}(t)
\end{gathered}
$$

for $(v, z) \in \mathcal{V} \times \mathcal{Z}$ subject to the constraints (6.4), (6.7) and

$$
D \hat{g}\left(\bar{y}, \mu_{0}\right)(z, d)=0 \quad \text { on } \overline{\mathcal{I}}_{b} \text {. }
$$

This last constraint is equivalent to $(6.5)-(6.6)$ since $D \hat{g}^{(1)}\left(\bar{u}, \bar{y}, \mu_{0}\right)(v, z, d)=\frac{\mathrm{d}}{\mathrm{d} t} D \hat{g}\left(\bar{y}(t), \mu_{0}\right)(z(t), d)$. Then, using the relation (6.12), we can show that $\pi_{d}$, the directional derivative of $p^{\mu}$ w.r.t. $\mu$, is the multiplier associated with (6.4) in formulation (6.14)-(6.15) of $\left(\mathcal{P}_{d}\right)$, and that the directional derivative of $\frac{\mathrm{d} \eta^{\mu}}{\mathrm{d} t}$ w.r.t. $\mu$, equal by $(6.13)$ to $\dot{\zeta}_{d}=-\dot{\zeta}_{1, d}$, is equal to the multiplier associated with the constraint (6.15).

Let us conclude this section by the following observation. For $i \in I_{\text {to }}$, since $\bar{t}_{i}^{\text {en }}=\bar{t}_{i}^{\mathrm{ex}}$, the optimality system of $\left(\mathcal{P}_{d}\right)$, easily obtained, yields that $H_{u u} v_{d}+H_{u y} z_{d}+\pi_{1, d} f_{u}=0$ at $\bar{t}_{i}^{\text {en }}$, and that the jump of $\pi_{1, d}$ is given by $\left[\pi_{1, d}\left(\bar{t}_{i}^{\mathrm{en}}\right)\right]=-\gamma_{d, i}^{1} g_{y}\left(\bar{y}\left(\bar{t}_{i}^{\mathrm{en}}\right)\right)$. Hence, the jump of $v_{d}$ is given by

$$
\left[v_{d}\left(\bar{t}_{i}^{\mathrm{en}}\right)\right]=\gamma_{d, i}^{1} H_{u u}^{-1}(\bar{u}, \bar{y}, \bar{p})\left(\bar{t}_{i}^{\mathrm{en}}\right) g_{y}\left(\bar{y}\left(\bar{t}_{i}^{\mathrm{en}}\right)\right) f_{u}(\bar{u}, \bar{y})\left(\bar{t}_{i}^{\mathrm{en}}\right)=\gamma_{d, i}^{1} H_{u u}^{-1}(\bar{u}, \bar{y}, \bar{p})\left(\bar{t}_{i}^{\mathrm{en}}\right) g_{u}^{(1)}(\bar{u}, \bar{y})\left(\bar{t}_{i}^{\mathrm{en}}\right),
$$

and we obtain from $(6.10)-(6.11)$

$$
\sigma_{d, i}^{\mathrm{ex}}-\sigma_{d, i}^{\mathrm{en}}=-\frac{g_{u}^{(1)}(\bar{u}, \bar{y})\left(\bar{t}_{i}^{\mathrm{en}}\right)\left[v_{d}\left(\bar{t}_{i}^{\mathrm{en}}\right)\right]}{\left.\frac{\mathrm{d}}{\mathrm{d} t} g^{(1)}(\bar{u}, \bar{y})\right|_{t=\bar{t}_{i}^{\mathrm{en}}}}=C_{i} \gamma_{d, i}^{1}, \quad \text { with } \quad C_{i}:=\frac{H_{u u}^{-1}(\bar{u}, \bar{y}, \bar{p})\left(\bar{t}_{i}^{\mathrm{en}}\right)\left(g_{u}^{(1)}(\bar{u}, \bar{y})\left(\bar{t}_{i}^{\mathrm{en}}\right)\right)^{2}}{-\left.\frac{\mathrm{d}}{\mathrm{d} t} g^{(1)}(\bar{u}, \bar{y})\right|_{t=\bar{t}_{i}^{\mathrm{en}}}}>0
$$

Since $\gamma_{d, i}^{1} \geq 0$ for $i \in I_{\text {to }}$, we see that $\sigma_{d, i}^{\mathrm{ex}}-\sigma_{d, i}^{\mathrm{en}} \geq 0$, with equality iff $\gamma_{d, i}^{1}=0$. It follows that, for $\mu-\mu_{0}=d$, the length of the boundary arc and the jump parameter are related, at first order, by

$$
t_{i}^{\mu, \mathrm{ex}}-t_{i}^{\mu, \mathrm{en}}=C_{i} \nu_{i}^{\mu, 1}+o\left(\left\|\mu-\mu_{0}\right\|\right)
$$

Remark 6.3. It was quite expected that nonessential touch points generally turn into boundary arcs for constraints of first order (see e.g. [9]). However it was surprising to be able to describe this transition between touch points and boundary arcs by a shooting approach when the structure is not stable, and obtain the differentiability of the shooting mapping, and in particular of the entry and exit times of the appearing boundary arcs.

Note that those results are false for control constraints. Consider for example the problem below:

$$
\min _{u \in \mathcal{U}} \int_{0}^{2}\left(u(t)-(t-1)^{2}\right)^{2} \mathrm{~d} t
$$

Here we have no state, or more precisely, the state is equal to the time. Obviously the solution is $u(t)=(t-1)^{2}$. Add now a constraint $u(t) \geq \varepsilon$ for $\varepsilon>0$. Then the optimal solution is $u(t)=\varepsilon$ on $\left[\tau_{-}^{\varepsilon}, \tau_{+}^{\varepsilon}\right]$ with $\tau_{ \pm}^{\varepsilon}=1 \pm \sqrt{\varepsilon}$, and $u(t)=(t-1)^{2}$ on $\left[0, \tau_{-}^{\varepsilon}\right) \cup\left(\tau_{+}^{\varepsilon}, 2\right]$. So for $\varepsilon>0$ a boundary arc appear, whose end points $\tau_{-}^{\varepsilon}$ and $\tau_{+}^{\varepsilon}$ are not differentiable at the point $\varepsilon=0$, and whose length is of order $\sqrt{\varepsilon}$ and not $\varepsilon$. A fortiori the shooting mapping is not differentiable at the point $\varepsilon=0$, and the algorithm described in Section 8 has no obvious extension to control constraints (or more generally to mixed control-state constraints). 


\section{EXAMPLE OF SENSITIVITY ANALYSIS}

We illustrate the results of this paper on a very basic example. We consider the problem of an elastic line of positive mass, fixed at its endpoints and submitted to a vertical uniform force $(g)$. The problem is to find the equilibrium position, i.e. minimize the energy. Assuming the elastic potential to be quadratic with unit constant, this can be written as the optimal control problem (with $t$ replaced by $x \in[0,1]$ ):

$$
\min \int_{0}^{1}\left(\frac{u(x)^{2}}{2}+g y(x)\right) \mathrm{d} x, \quad \dot{y}(x)=u(x), \quad y(0)=0=y(1) .
$$

We add a first-order state constraint, e.g. the level of the floor

$$
y(x) \geq-h .
$$

Here $g$ and $h$ denotes positive constants.

Remark 7.1. Our results can be extended with only slight adaptations to the case when there are also finitely many equality and inequality constraints on the final state, if we assume in addition a controllability condition. In the case of a fixed final state, $y(T)=y_{T}$ given in $\mathbb{R}^{n}$, this controllability condition is assumption (A1') below. Recall that given $\delta>0$, we denote by $\Omega^{\delta}:=\{t \in[0, T]$, $\operatorname{dist}\{t ; I(g(\bar{y}))\}<\delta\}$.

(A1') (i) The initial and final conditions satisfy $g\left(y_{0}\right)<0$ and $g\left(y_{T}\right)<0$.

(ii) There exists $\delta>0$ such that the linear mapping $\mathcal{U} \rightarrow W^{1, \infty}\left(\Omega^{\delta}\right) \times \mathbb{R}^{n} ; v \mapsto\left(\left.g_{y}(\bar{y}(\cdot)) z_{v}(\cdot)\right|_{\Omega^{\delta}}, z_{v}(T)\right)$, where $z_{v}$ is the solution of (1.24) and $\left.\right|_{\Omega^{\delta}}$ denotes the restriction to the set $\Omega^{\delta}$, is onto (and therefore has a bounded right inverse by the open mapping theorem).

This assumption (A1') plays the role of Lemma 1.2 in the proofs. Note that when the dynamics $f$ is linear, i.e. $f(u, y)=A y+B u$, then (A1')(ii) is satisfied if the pair $(A, B)$ is controllable, and if (A1')(i) and (A3) hold.

For the example considered here, (A1') is obviously satisfied so all the previous results are valid. The unconstrained optimal trajectory when $h / g \geq 1 / 8$ is given by:

$$
y(x)=\frac{1}{2} g x^{2}-\frac{1}{2} g x, \quad u(x)=g x-\frac{1}{2} g .
$$

The resolution of the constrained problem when $h / g \leq 1 / 8$ is as follows. The trajectory is:

$$
\begin{gathered}
u(x)= \begin{cases}g\left(x-x_{\mathrm{en}}\right) & \text { on }\left[0, x_{\mathrm{en}}\right] \\
0 & \text { on }\left[x_{\mathrm{en}}, x_{\mathrm{ex}}\right] \\
g\left((x-1)-\left(x_{\mathrm{ex}}-1\right)\right) & \text { on }\left[x_{\mathrm{ex}}, 1\right]\end{cases} \\
y(x)= \begin{cases}g\left(x^{2} / 2-x_{\mathrm{en}} x\right) & \text { on }\left[0, x_{\mathrm{en}}\right] \\
-h & \text { on }\left[x_{\mathrm{en}}, x_{\mathrm{ex}}\right] \\
g\left((x-1)^{2} / 2-\left(x_{\mathrm{ex}}-1\right)(x-1)\right) & \text { on }\left[x_{\mathrm{ex}}, 1\right] .\end{cases}
\end{gathered}
$$

Entry and exit positions $x_{\mathrm{en}}$ and $x_{\mathrm{ex}}$ are given by:

$$
x_{\mathrm{en}}=\sqrt{2 h / g}, \quad x_{\mathrm{ex}}=1-\sqrt{2 h / g} .
$$

The alternative state constraint multiplier on $\left[x_{\mathrm{en}}, x_{\mathrm{ex}}\right]$ is given by:

$$
\eta_{1}(x)=p_{1}(x)=-g\left(x-x_{\mathrm{ex}}\right) \geq 0, \quad \dot{\eta}_{1}(x)=-g<0,
$$

and hence, the jump parameter at entry time is:

$$
\nu_{\mathrm{en}}^{1}=\eta_{1}\left(x_{\mathrm{en}}\right)=g\left(x_{\mathrm{ex}}-x_{\mathrm{en}}\right)=g(1-2 \sqrt{2 h / g}) \geq 0 .
$$


We consider perturbations w.r.t. nominal values of parameters $g=g_{0}=1$ and $h=h_{0}=1 / 8$, for which there is a touch point at $x=1 / 2$. The strong sufficient second-order condition (3.8) clearly holds, since the linear-quadratic problem:

$$
\min \int_{0}^{1} \frac{v^{2}(x)}{2} \mathrm{~d} x, \quad \dot{z}(x)=v(x), \quad z(0)=0=z(1)
$$

having a strongly convex cost function, has $(v, z)=0$ for unique solution. Let us then study the perturbed quadratic problem at $\left(g_{0}, h_{0}\right)$ in direction $d:=(\gamma, \eta)$ :

$$
\min \int_{0}^{1}\left(\frac{v(x)^{2}}{2}-\gamma z(x)\right) \mathrm{d} x, \quad \dot{z}(x)=v(x), \quad z(0)=0=z(1),
$$

subject to the interior point inequality constraint:

$$
z(1 / 2) \geq-\eta
$$

The unconstrained trajectory is:

$$
z_{d}(x)=\gamma\left(\frac{x^{2}}{2}-\frac{x}{2}\right), \quad v_{d}(x)=\gamma\left(x-\frac{1}{2}\right) .
$$

Therefore, the constraint is active, iff $\eta \leq \gamma / 8$. If $\eta>\gamma / 8,(7.7)$ corresponds to the directional derivative of the unconstrained trajectory (7.3). When $\eta \leq \gamma / 8$, the constraint (7.6) is active, i.e. $z_{d}(1 / 2)=-\eta$, and therefore, the solution of the linear-quadratic problem is as follows:

$$
\begin{gathered}
v_{d}(x)= \begin{cases}\gamma x-(2 \eta+\gamma / 4) & \text { on }[0,1 / 2] \\
\gamma(x-1)+(2 \eta+\gamma / 4) & \text { on }[1 / 2,1] .\end{cases} \\
z_{d}(x)= \begin{cases}\gamma x^{2} / 2-(2 \eta+\gamma / 4) x & \text { on }[0,1 / 2] \\
\gamma(x-1)^{2} / 2+(2 \eta+\gamma / 4)(x-1) & \text { on }[1 / 2,1] .\end{cases}
\end{gathered}
$$

The multiplier $\lambda_{d}$ associated with the constraint (7.6) is, by (6.16):

$$
\lambda_{d}=\left[\pi_{d}(1 / 2)\right]=-\left[v_{d}(1 / 2)\right]=-2(2 \eta-\gamma / 4) \geq 0,
$$

and, by (6.10)-(6.11), the variations of entry and exit points $\sigma_{d, \text { en }}$ and $\sigma_{d, \text { ex }}$ are given by:

$$
\sigma_{d, \mathrm{en}}=-\frac{v\left(1 / 2^{-}\right)}{g_{0}}=-\gamma / 4+2 \eta, \quad \sigma_{d, \mathrm{ex}}=-\frac{v\left(1 / 2^{+}\right)}{g_{0}}=\gamma / 4-2 \eta .
$$

By (7.5) and (7.4), we check that the above formula corresponds to the first-order variations, with $g=g_{0}+\gamma$ and $h=h_{0}+\eta,|\gamma|,|\eta|$ small, of:

$$
\nu_{\mathrm{en}}^{1}=(1+\gamma)\left(1-2 \sqrt{\frac{1 / 4+2 \eta}{1+\gamma}}\right), \quad x_{\mathrm{en}}=\sqrt{\frac{1 / 4+2 \eta}{1+\gamma}}, \quad x_{\mathrm{ex}}=1-\sqrt{\frac{1 / 4+2 \eta}{1+\gamma}} .
$$

We consider perturbations in three directions $d=(\gamma, \eta)$ :

$$
\begin{array}{ll}
\text { Case (a) } & (\gamma, \eta)=(0,-0.02) \\
\text { Case (b) } & (\gamma, \eta)=(1,0) \\
\text { Case (c) } & (\gamma, \eta)=(1,-0.02)
\end{array}
$$



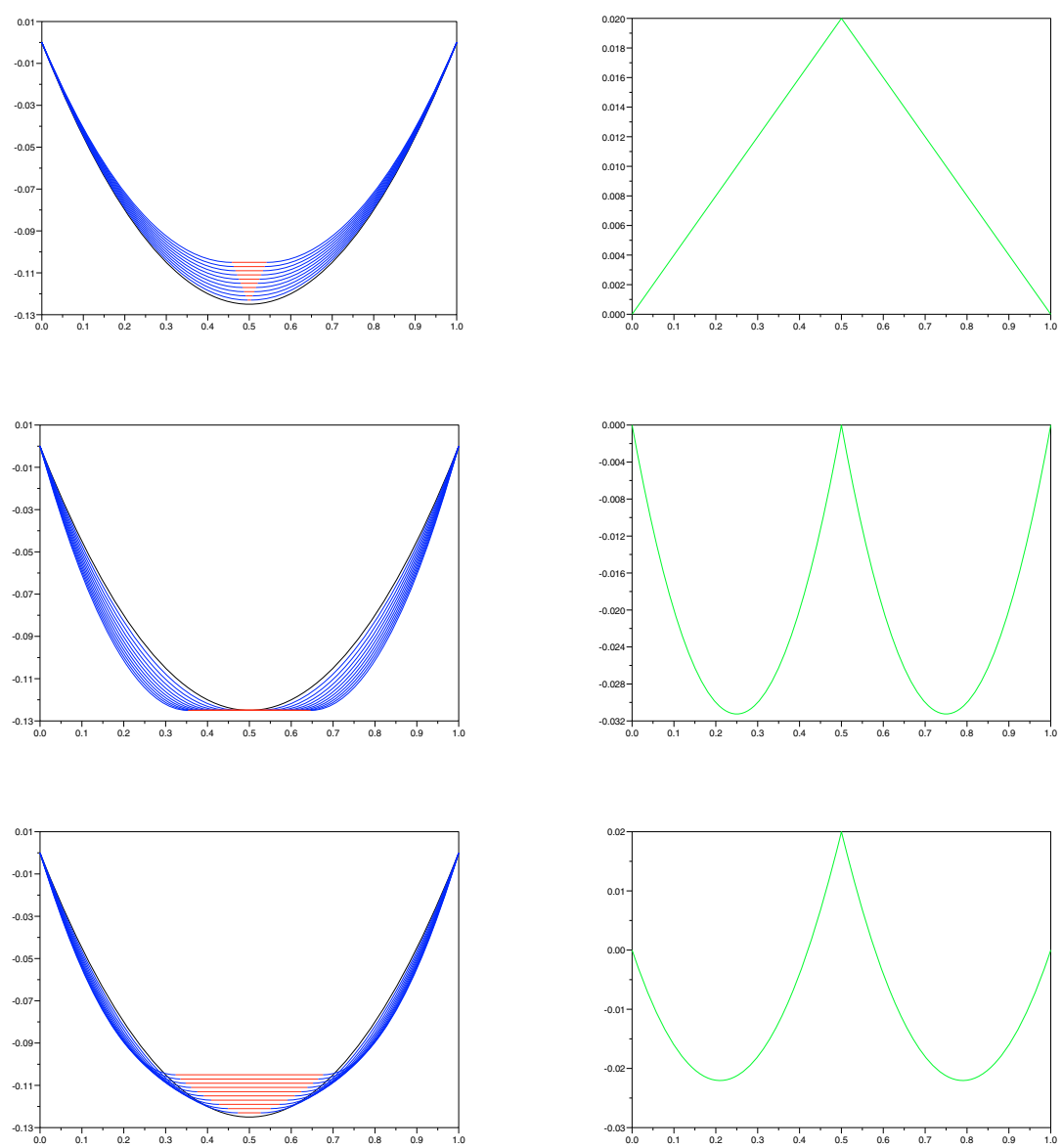

Figure 1. Perturbation of the state (left) and directional derivatives (right) in case (a) to (c) (from top to bottom).

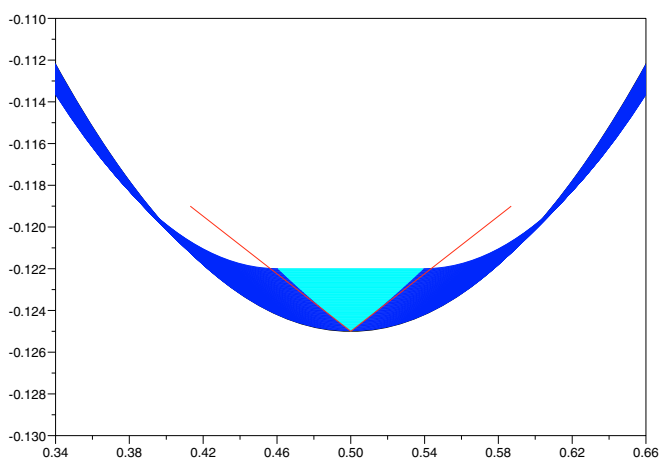

FiguRE 2. Variation of the length of the boundary arc in case (c). 
Case (a) corresponds to an elevation of the ground level, case (b) corresponds to an increasing of the "gravitational" force $g$, both of them leading to the emergence of a boundary arc, and case (c) combines elevation of the ground and increasing of $g$. The perturbed trajectories and directional derivatives of the state in $W^{1, r}$, $1 \leq r<+\infty$, are presented for each case in Figure 1. The unconstrained trajectory for $\left(g_{0}, h_{0}\right)$ is a parabola. In Figure 2, we focus on the appearance of the boundary arc in case (c), check that its length is of the order of the perturbation and compare with the directional derivatives of variation of junction times (7.9).

\section{8. Номотору METHOD}

We present in this section an algorithm that combines shooting and continuation (or homotopy) methods for solving optimal control problems with a scalar first-order state constraint, when the structure of the trajectory is unknown. It keeps the advantages of shooting methods regarding to the (high) precision and the (low) complexity, and enables to get rid of the (sometimes) hard task to guess a priori the structure of the trajectory, and of the initialization of some of the shooting parameters (only the initialization of the initial costate is left to the user). The idea is to handle automatically the appearance (and disappearance) of boundary arcs, so that the algorithm finds itself the structure of the trajectory. The results of the previous sections are used.

General results on homotopy methods can be found in e.g. [1], [11], Chapter 5, and applications of homotopy methods to optimal control problems in e.g. [10,14,24].

\subsection{Description of the algorithm}

The problem to be solved is the following:

$$
\begin{aligned}
\min _{(u, y) \in \mathcal{U} \times \mathcal{Y}} & \int_{0}^{T} \ell(u(t), y(t)) \mathrm{d} t+\phi(y(T)) \\
\text { subject to } & \dot{y}(t)=f(u(t), y(t)) \quad \text { a.e. on }[0, T], \quad y(0)=y_{0}, \\
& g(y(t)) \leq 0 \quad \text { on }[0, T] .
\end{aligned}
$$

We assume that $(\mathcal{P})$ satisfies $(\mathrm{A} 0)-(\mathrm{A} 1)$. In view of Remark 7.1, we can more generally consider a fixed final state $y(T)=y_{T}$ and $\phi=0$ if we assume in addition that the controllability condition (A1') holds.

We consider the natural homotopy on the state constraint $\left(\mathcal{P}^{\mu}\right)$, for $\mu \in[0,1]$, defined by $\left(\ell^{\mu}, \phi^{\mu}, f^{\mu}, y_{0}^{\mu}\right):=$ $\left(\ell, \phi, f, y_{0}\right)$ and

$$
g^{\mu}(y):=g(y)-(1-\mu) K,
$$

where the constant $K>0$ is large enough, so that the state constraint of problem $\left(\mathcal{P}^{0}\right)$ is not active, except maybe at finitely many (isolated) touch points in $(0, T)$. We explain later how we choose $K$ in the algorithm. We thus have $\left(\mathcal{P}^{1}\right) \equiv(\mathcal{P})$.

The shooting mapping (4.39) for $\left(\mathcal{P}^{\mu}\right)$ is denoted by $F(\theta, \mu)$, where $\theta$ is the vector of shooting parameters, of variable dimension depending on the structure of the trajectory, and $\mu$ is the (scalar) homotopy parameter. Since we only have here one state constraint of first order, note that the structure of the trajectory, and hence $F$, is entirely determined by the dimension of $\theta$. More precisely, the number of boundary arcs of the trajectory $N_{\text {ba }}$ is given by (assuming the state constraint inactive at initial and final times)

$$
N_{\mathrm{ba}}=\frac{\operatorname{dim}(\theta)-n}{3} \in \mathbb{N} .
$$

The structure of the trajectory follows then from the alternation between interior and boundary arcs. We denote by $y^{\theta, \mu}$ the state solution of the alternative formulation for the shooting parameter $\theta$ and the value of the homotopy parameter $\mu$. The algorithm is as follows (see Algorithm 8.1).

The algorithm is initialized by solving the unconstrained problem (without the state constraint) (8.1)-(8.2). We thus obtain a vector of shooting parameters $\theta_{0}$ (reduced to the initial costate), associated with a stationary 
point of (8.1)-(8.2), which is a local solution of (8.1)-(8.2) if the second-order sufficient condition (3.6) holds. The constant $K$ in (8.4) is taken equal to $K:=\max _{t \in[0, T]} g\left(y^{\theta_{0}, 0}(t)\right)$. If $K \leq 0$, then $\theta_{0}$ is a vector of shooting parameters associated with a local solution of $(\mathcal{P})$. If $K>0$, we start the homotopy from $\mu=\mu_{0}:=0$ in $(8.4)$ to $\mu=1$.

The variable $m_{k}$ denotes the maximum of $g^{\mu_{k}}\left(y^{\theta_{k}, \mu_{k}}\right)$, attained at time $\tau_{k}$. If $m_{k}$ is positive, this means that the state constraint is violated so the structure is not correct and we have to add a boundary arc (Step A). The variable $i_{k}$ equals zero iff all entry and exit times of boundary arcs are such that entry times are lower than or equal to the corresponding exit times, and equals $i>0$ if the entry time of the $i$ th boundary arc is greater than the corresponding exit time. If $i_{k}=i>0$, the structure is not correct again so we have to delete the $i$ th boundary arc (Step A). All this will be justified later in Section 8.3 under some assumptions. If both $m_{k} \leq 0$ and $i_{k}=0$, this means that the structure is correct, i.e. the current iterate $\theta_{k}$ is a vector of shooting parameters associated with a stationary point $\left(u^{\mu_{k}}, y^{\mu_{k}}\right)$ of $\left(\mathcal{P}^{\mu_{k}}\right)$. We thus increase the value of $\mu$ and do a simple predictor-corrector iteration (Steps B-C), keeping the same structure for the shooting mapping. Then in Step D we calculate the new values of $m_{k+1}$ and $i_{k+1}$ that say whether the structure is still correct or has to be updated in the next iteration. We do so until reaching the value $\mu=1$.

If the Newton algorithm in Step C fails, then we decrease the value of the step $\Delta \mu_{k}$, and go back to the last value $\left(\mu_{k-1}, \theta_{k-1}\right)$ satisfying $F\left(\mu_{k-1}, \theta_{k-1}\right)=0$ and $\max \left(m_{k-1}, i_{k-1}\right)=0$.

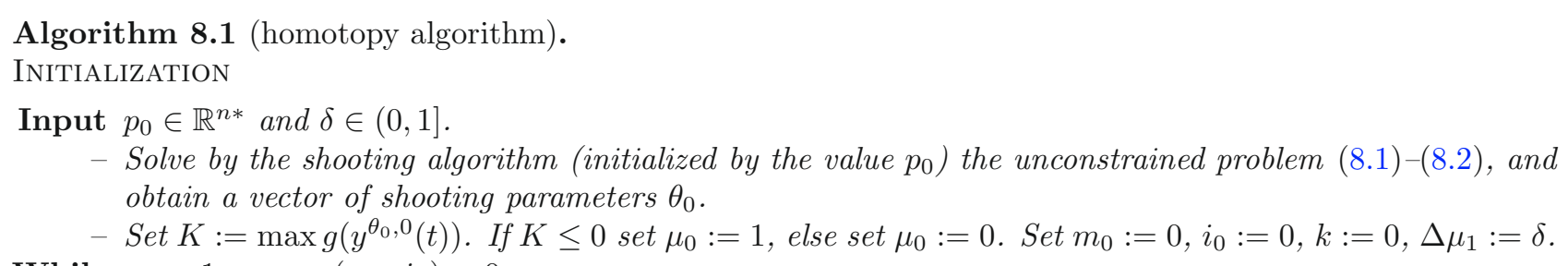

While $\mu_{k}<1$ or $\max \left(m_{k}, i_{k}\right)>0$

If $\max \left(m_{k}, i_{k}\right)>0$ then STEP A (update the structure)

IF $m_{k}>0$ THEN (addition of a boundary arc)

Initialize the new shooting parameters $\left(\nu^{1}, \tau_{\mathrm{en}}, \tau_{\mathrm{ex}}\right)$ associated with this boundary arc by:

$$
\nu^{1}=0 \quad \text { and } \quad \tau_{\mathrm{en}}=\tau_{\mathrm{ex}}=\tau_{k} .
$$

Take the remaining shooting parameters equal to the previous value $\theta_{k}$, and obtain a vector of shooting parameters $\bar{\theta}_{k}$ of dimension $\operatorname{dim}\left(\theta_{k}\right)+3$.

END IF

IF $i_{k}>0$ (suppression of a boundary arc)

Remove the shooting parameters $\left(\nu^{1}, \tau_{\mathrm{en}}, \tau_{\mathrm{ex}}\right)$ corresponding to the $i_{k}$-th boundary arc from the vector of shooting parameters $\theta_{k}$, and obtain a new vector of shooting parameters $\bar{\theta}_{k}$ of dimension $\operatorname{dim}\left(\theta_{k}\right)-3$.

END IF

Set $\bar{\mu}_{k}:=\mu_{k}$ (the value of $\mu$ is unchanged by this step).

Else STEP B (prediction)

Set $k:=k+1$

$\bar{\mu}_{k}:=\min \left\{\mu_{k-1}+\Delta \mu_{k} ; 1\right\}$

End if

$\bar{\theta}_{k}:=\theta_{k-1}-D_{\theta} \mathcal{F}\left(\theta_{k-1}, \mu_{k-1}\right)^{-1} D_{\mu} \mathcal{F}\left(\theta_{k-1}, \mu_{k-1}\right)\left(\bar{\mu}_{k}-\mu_{k-1}\right)$.

STEP C (correction) Try to solve, by a Newton method, $\mathcal{F}\left(\theta, \bar{\mu}_{k}\right)=0$. The Newton algorithm is initialized by the value $\bar{\theta}_{k}$.

If the Newton algorithm fails then (go back to old values of $\mu$ and $\theta$ and decrease the step). Set $\mu_{k}:=$ $\mu_{k-1}, \theta_{k}:=\theta_{k-1}, m_{k}:=m_{k-1}, i_{k}:=i_{k-1}, \tau_{k}:=\tau_{k-1}, \Delta \mu_{k}:=\Delta \mu_{k} / 2$ and $k:=k-1$.

Else (success) obtain a solution $\theta_{k}$ such that $F\left(\theta_{k}, \bar{\mu}_{k}\right)=0$. Set $\mu_{k}:=\bar{\mu}_{k}$. 
STEP D (verify if the structure is correct)

- Set $m_{k}:=\max g^{\mu_{k}}\left(y^{\theta_{k}, \mu_{k}}(t)\right)$ and $\tau_{k} \in \operatorname{argmax} g^{\mu_{k}}\left(y^{\theta_{k}, \mu_{k}}(t)\right)$.

- Set $i_{k}:=0$. For all $i=1, \ldots, N_{\mathrm{ba}}^{k}\left(N_{\mathrm{ba}}^{k}\right.$ given by (8.5)), if $\theta_{k}$ is such that the entry time corresponding to the ith boundary arc is greater than the exit time corresponding to the ith boundary arc, then $i_{k}:=i$.

End if

- IF $\max \left(m_{k}, i_{k}\right)=0$ THEN set $\Delta \mu_{k+1}:=\delta$.

\section{End while}

Remark 8.2. Note that the Newton algorithm converges quadratically, provided that the initial point is good enough. Therefore, we can see rapidly in Step C whether the Newton algorithm converges or not and if we need to decrease the step $\Delta \mu_{k}$.

Remark 8.3. Clearly, the present algorithm does not take into account all possible events, since it principally assumes the stability of boundary arcs (which holds when uniform strict complementarity is satisfied, see assumption $\left(\mathcal{H}_{2}\right)$ below). If uniform strict complementarity does not hold along the homotopy path, then it may happen for example that a boundary arc splits into two boundary arcs, or on the contrary that two boundary arcs melt into one.

\subsection{Existence of the homotopy path}

Assume that the following holds:

$\left(\mathcal{H}_{0}\right)$ For $\mu_{0}=0$, the unconstrained problem $\left(\mathcal{P}^{0}\right)$ has a local solution $(\bar{u}, \bar{y})$ that satisfies $(\mathrm{A} 0)-(\mathrm{A} 3)$, the contact set $I\left(g^{0}(\bar{y})\right)$ is composed of finitely many (nonessential) touch points in $(0, T)$, all of them satisfying (1.26), and the strong second-order sufficient condition (3.8) is satisfied.

By Theorem 3.3, $\left(\mathcal{H}_{0}\right)$ implies that there exists $\tilde{\mu}>0$ such that for all $\mu \in[0, \tilde{\mu}),\left(\mathcal{P}^{\mu}\right)$ has a locally unique local solution $\left(u^{\mu}, y^{\mu}\right)$ with multipliers $\left(p^{\mu}, \eta^{\mu}\right)$, that satisfies assumptions (A1)-(A3) for $\left(\mathcal{P}^{\mu}\right)$. In addition, this local solution $\left(u^{\mu}, y^{\mu}\right)$ of $\left(\mathcal{P}^{\mu}\right)$ has a neighboring structure to that of $(\bar{u}, \bar{y})$, implying that if $(\bar{u}, \bar{y})$ has $N$ touch points, then $\left(u^{\mu}, y^{\mu}\right)$ has at most $N$ boundary arcs or touch points, i.e. satisfies (A4). Further, strict complementarity holds on the boundary arcs of $\left(u^{\mu}, y^{\mu}\right)$, and the touch points satisfy (1.26) by continuity, i.e. (A5)-(A6) are satisfied. Finally, $\left(u^{\mu}, y^{\mu}\right)$ satisfies the strong second-order condition (3.8) for $\left(\mathcal{P}^{\mu}\right)$ by Lemma 3.4.

Consequently, assumption $\left(\mathcal{H}_{0}\right)$ ensures that the homotopy path is well-defined on an interval $[0, \tilde{\mu}) \subset[0,1]$, and that assumptions (A1)-(A6) as well as the strong second-order sufficient condition (3.8) remain satisfied on this neighborhood. Let

$$
\begin{array}{r}
\mu_{\max }:=\sup \left\{\tilde{\mu} \in[0,1]: \text { for all } \mu \in[0, \tilde{\mu}], \text { the locally unique local solution }\left(u^{\mu}, y^{\mu}\right)\right. \\
\text { of } \left.\left(\mathcal{P}^{\mu}\right) \text { satisfies }(\mathrm{A} 1)-(\mathrm{A} 6) \text { and }(3.8)\right\} .
\end{array}
$$

The preceding discussion shows that assumption $\left(\mathcal{H}_{0}\right)$ implies that $\mu_{\max }>0$.

Lemma 8.4 (existence of the homotopy path). Assume that $\left(\mathcal{H}_{0}\right)$ holds, that there exists $L>0$ such that for all $\mu \in\left[0, \mu_{\max }\right)$,

$$
\left\|\dot{u}^{\mu}\right\|_{1}+\left\|u^{\mu}\right\|_{\infty} \leq L
$$

and that (A1) and (A3) are uniformly satisfied, i.e. there exist $\beta, \varepsilon, \zeta>0$ such that for all $\mu \in\left[0, \mu_{\max }\right)$,

$$
g^{\mu}\left(y_{0}^{\mu}\right)<-\zeta \quad \text { and } \quad\left|\left(g^{\mu}\right)_{u}^{(1)}\left(u^{\mu}(t), y^{\mu}(t)\right)\right| \geq \beta, \quad \text { for all } t, \operatorname{dist}\left\{t ; I\left(g^{\mu}\left(y^{\mu}\right)\right)\right\} \leq \varepsilon \text {. }
$$

Then there exists a sequence $\left(\mu_{n}\right)_{n \in \mathbb{N}^{*}}$ such that $\mu_{n} \uparrow \mu_{\max },\left(u^{\mu_{n}}, y^{\mu_{n}}\right) \rightarrow(\tilde{u}, \tilde{y})$ uniformly, $\left(p^{\mu_{n}}, \mathrm{~d} \eta^{\mu_{n}}\right)$ weakly-* converges to $(\tilde{p}, \mathrm{~d} \tilde{\eta})$ in $L^{\infty}\left(0, T ; \mathbb{R}^{n *}\right) \times \mathcal{M}[0, T]$, and $(\tilde{u}, \tilde{y}, \tilde{p}, \tilde{\eta})$ is a stationary point and its multipliers of $\left(\mathcal{P}^{\mu_{\max }}\right)$.

Moreover, if $(\tilde{u}, \tilde{y}, \tilde{p}, \tilde{\eta})$ satisfies assumptions (A1)-(A6) and the strong second-order sufficient condition (3.8), then $\left(u^{\mu}, y^{\mu}, p^{\mu}, \eta^{\mu}\right)$ converges when $\mu \uparrow \mu_{\max }$ to a locally unique local solution of $\left(\mathcal{P}^{\mu_{\max }}\right)$ and its multipliers 
$(\tilde{u}, \tilde{y}, \tilde{p}, \tilde{\eta})=:\left(u^{\mu_{\max }}, y^{\mu_{\max }}, p^{\mu_{\max }}, \eta^{\mu_{\max }}\right)$, and $\mu_{\max }=1$, i.e. the homotopy path is locally well-defined over $\mu \in[0,1]$.

Proof. Consider a sequence $\left(\mu_{n}\right)_{n \in \mathbb{N}^{*}} \subset\left[0, \mu_{\max }\right)$ such that $\mu_{n} \rightarrow \mu_{\max }$ when $n \rightarrow+\infty$. Since $W^{1,1}(0, T)$ is compactly embedded in $C^{0}[0, T],(8.7)$ implies that there exists a subsequence, still denoted by $\left(\mu_{n}\right)$, such that the sequence $\left(u^{\mu_{n}}\right)$ converges uniformly to some $\tilde{u} \in \mathcal{U}$. By (8.7), we may pass to the limit in the state equation (1.2) and obtain that $y^{\mu_{n}}$ converges in $\mathcal{Y}$ to the state $\tilde{y}:=y_{\tilde{u}}^{\mu_{\max }}$ solution of (1.10).

By (8.8), Robinson's constraint qualification (1.17) is uniformly satisfied for all $\mu \in\left[0, \mu_{\max }\right)$, i.e. the positive constant $\gamma$ in (1.17) does not depend on $\mu$. It follows then from [8], Proposition 4.43, and (8.7) that $\left\|\mathrm{d} \eta^{\mu_{n}}\right\|_{\mathcal{M}[0, T]}$ is uniformly bounded. Therefore there exists a weakly-* convergent subsequence $\mathrm{d} \eta^{\mu_{n}} \stackrel{*}{\rightarrow} \mathrm{d} \tilde{\eta}$ in $\mathcal{M}[0, T]$. Since $\mathrm{d} \eta^{\mu} \in N_{K}\left(g^{\mu}\left(y^{\mu}\right)\right)$ for all $\mu \in\left[0, \mu_{\max }\right)$, and $g^{\mu_{n}}\left(y^{\mu_{n}}\right) \rightarrow g^{\mu_{\max }}(\tilde{y})$ strongly (i.e. uniformly), we deduce easily from the definition of the normal cone that $\mathrm{d} \tilde{\eta} \in N_{K}\left(g^{\mu_{\max }}(\tilde{y})\right)$. By the costate equation (1.13) (with $\alpha=1), \mathrm{d} p^{\mu}$ is uniformly bounded in $\mathcal{M}\left([0, T] ; \mathbb{R}^{n *}\right)$. Therefore, there exists a weakly-* convergent subsequence $\mathrm{d} p^{\mu_{n}} \stackrel{*}{\rightarrow} \mathrm{d} \tilde{p} \in \mathcal{M}\left([0, T] ; \mathbb{R}^{n *}\right)$. Due to the convergence of the final condition (1.14), we deduce easily from the integration by parts formula [13], p. 154

$$
\int_{0}^{T} p(t) \varphi(t) \mathrm{d} t=-\int_{0}^{T} \mathrm{~d} p(t) \Phi(t)+p(T) \Phi(T) \quad \text { for all }(p, \varphi) \in B V \times L^{1} \text { with } \Phi(t):=\int_{0}^{t} \varphi(s) \mathrm{d} s
$$

that $p^{\mu_{n}}$ weakly-* converges in $L^{\infty}\left(0, T ; \mathbb{R}^{n *}\right)$ to a limit $\tilde{p}$ given by $\tilde{p}(t):=\int_{T}^{t} \mathrm{~d} \tilde{p}(s)+\phi_{y}^{\mu_{\max }}(\tilde{y}(T))$. Since (1.18) and (1.13) are linear in $p$ and $\eta$, we may pass to the weak-* limit and obtain that $(\tilde{u}, \tilde{y})$ is a stationary point of $\left(\mathcal{P}^{\mu_{\max }}\right)$ with multipliers $(\tilde{p}, \tilde{\eta})$.

Now assume that this stationary point $(\tilde{u}, \tilde{y})$ of $\left(\mathcal{P}^{\mu_{\max }}\right)$ satisfies assumptions $(\mathrm{A} 1)-(\mathrm{A} 6)$ and the strong second-order sufficient condition (3.8). These assumptions imply by Theorem 3.3 that $(\tilde{u}, \tilde{y})$ is an isolated stationary point of $\left(\mathcal{P}^{\mu_{\max }}\right)$, which shows the local uniqueness of the stationary point $(\tilde{u}, \tilde{y})$ of $\left(\mathcal{P}^{\mu_{\max }}\right)$ constructed above and of its multipliers. In addition $(\tilde{u}, \tilde{y})$ is a local solution of $\left(\mathcal{P}^{\mu_{\max }}\right)$, and by Theorem 3.3, we obtain the existence of the homotopy path on the interval $\left[\mu_{\max }, \mu_{\max }+\varepsilon\right)$, for some $\varepsilon>0$, and assumptions (A1)-(A6) hold on this interval by Theorem 3.3, as well as the strong second-order condition (3.8) by Lemma 3.4. This implies that $\mu_{\max }=1$, otherwise this would contradict the definition of $\mu_{\max }$. Therefore the homotopy path is locally well-defined over $[0,1]$.

We thus make the assumptions below:

$\left(\mathcal{H}_{1}\right)$ For all $\mu \in[0,1],\left(u^{\mu}, y^{\mu}\right)$ satisfies (A2), there exist $L>0$ and $\beta, \varepsilon, \zeta>0$ such that (8.7) and (8.8) hold, and $g^{\mu}\left(y^{\mu}(T)\right)<0$.

$\left(\mathcal{H}_{2}\right)$ For all $\mu \in[0,1],\left(u^{\mu}, y^{\mu}\right)$ has finitely many boundary arcs, and there exists $\beta>0$ such that for all $\mu \in[0,1], \dot{\eta}_{1}^{\mu}<-\beta$ on the boundary $\operatorname{arcs}$ of $\left(u^{\mu}, y^{\mu}\right)$ (with $\eta_{1}^{\mu}$ the alternative state constraint multiplier associated with $\left.\left(u^{\mu}, y^{\mu}\right)\right)$.

$\left(\mathcal{H}_{3}\right)$ For all $\mu \in[0,1],\left(u^{\mu}, y^{\mu}\right)$ has finitely many (nonessential) touch points, all of them satisfying (1.26).

$\left(\mathcal{H}_{4}\right)$ For all $\mu \in[0,1],\left(u^{\mu}, y^{\mu}\right)$ satisfies the strong second-order sufficient condition (3.8) for $\left(\mathcal{P}^{\mu}\right)$.

Actually the Algorithm 8.1 is correct only if we replace assumption $\left(\mathcal{H}_{3}\right)$ by:

$\left(\mathcal{H}_{3}^{\prime}\right)$ For all $\mu \in[0,1],\left(u^{\mu}, y^{\mu}\right)$ has at most one (nonessential) touch point, and the latter satisfies (1.26). But the algorithm can be generalized to the more general case case when $\left(\mathcal{H}_{3}\right)$ holds (see Rem. 8.14).

Remark 8.5. Assumptions $\left(\mathcal{H}_{0}\right)-\left(\mathcal{H}_{4}\right)$ needed to ensure the existence (and local uniqueness) of the homotopy path, and the convergence of the algorithm, are rather strong, but they also give some indications on why the algorithm fails, if it fails (for other reasons than numerical ones, see Rem. 8.13). Either (8.7) is not satisfied (i.e. $u^{\mu}$ is not uniformly Lipschitz continuous), or the problem becomes singular (i.e. (8.8) fails), or a solution with infinitely many boundary arcs or touch points is met during the homotopy, or strict complementarity on boundary arcs fails, or finally the strong second-order sufficient condition (3.8) fails. 


\subsection{Correctness of the algorithm}

The existence of a locally unique local solution $\left(u^{\mu}, y^{\mu}\right)$ of $\left(\mathcal{P}^{\mu}\right)$, for all $\mu \in[0,1]$, is guaranteed by assumptions $\left(\mathcal{H}_{1}\right)-\left(\mathcal{H}_{4}\right)$. In addition, for all $\mu \in[0,1]$, the locally unique local solution $\left(u^{\mu}, y^{\mu}\right)$ of $\left(\mathcal{P}^{\mu}\right)$ has finitely many boundary arcs and touch points. So to prove the correctness of the algorithm, it suffices to show that the algorithm does find, in finitely many steps, these local solutions $\left(u^{\mu}, y^{\mu}\right)$ for a finite increasing sequence of values of $\mu$, until $\mu=1$ (in fact, the algorithm gives the vector of shooting parameters $\theta^{\mu}$, of appropriate dimensions, associated with the trajectory $\left.\left(u^{\mu}, y^{\mu}\right)\right)$. For this Lemmas 8.6 to 8.10 given below will be useful.

Lemma 8.6. Assume that $\left(\mathcal{H}_{0}\right)-\left(\mathcal{H}_{4}\right)$ hold. Then the trajectories $\left(u^{\mu}, y^{\mu}\right)_{\mu \in[0,1]}$ have finitely many different structures, and the mapping $\mu \mapsto \theta^{\mu}$ is globally Lipschitz continuous over $[0,1]$.

Here, since the dimension of $\theta^{\mu}$ may vary, by "globally Lipschitz continuous" we mean that on any subinterval of $[0,1]$ where the trajectories $\left(u^{\mu}, y^{\mu}\right)$ have "neighboring structures", then the mapping $\mu \mapsto \theta^{\mu}$ is Lipschitz continuous with a Lipschitz constant uniform on $[0,1]$.

Proof. By assumptions $\left(\mathcal{H}_{1}\right)-\left(\mathcal{H}_{4}\right)$ and Theorem 3.3, for all $\mu \in[0,1]$, there exists an open neighborhood $V_{\mu}$ of $\mu$ such that for all $\mu^{\prime} \in V_{\mu}$, the locally unique local solution $\left(u^{\mu^{\prime}}, y^{\mu^{\prime}}\right)$ of $\left(\mathcal{P}^{\mu^{\prime}}\right)$ has a neighboring structure to that of $\left(u^{\mu}, y^{\mu}\right)$, and the mapping $\mu^{\prime} \mapsto \theta^{\mu^{\prime}}$ is Lipschitz continuous over $V_{\mu}$. We can thus extract from $\left(V_{\mu}\right)_{\mu \in[0,1]}$ a finite covering $\left(V_{\hat{\mu}_{k}}\right)_{k=0, \ldots, M}$ of $[0,1]$. Since for each $\hat{\mu}_{k}$, there exist finitely many possible neighboring structures to that of $\left(u^{\hat{\mu}_{k}}, y^{\hat{\mu}_{k}}\right)$, and $\mu \mapsto \theta^{\mu}$ is Lipschitz continuous on each $V_{\hat{\mu}_{k}}$, the result follows.

Although by Lemma 8.6 the trajectories $\left(u^{\mu}, y^{\mu}\right)_{\mu \in[0,1]}$ have finitely many different structures, assumptions $\left(\mathcal{H}_{0}\right)-\left(\mathcal{H}_{4}\right)$ do not imply that there are finitely many changes in the structure of the trajectory along the homotopy path (see Rem. 8.7 below). More precisely, we say that the structure of the trajectory changes at $\bar{\mu} \in[0,1)$, if $\left(u^{\bar{\mu}}, y^{\bar{\mu}}\right)$ has a touch point that either disappears or turns into a boundary arc (of positive length) when $\mu \rightarrow \bar{\mu}^{+}$. We will therefore make the following assumption in the proof of correctness of the algorithm (Prop. 8.11), in addition to $\left(\mathcal{H}_{0}\right)-\left(\mathcal{H}_{4}\right)$ that ensure the existence of the homotopy path.

$\left(\mathcal{H}_{5}\right)$ There exist finitely many values of $\mu \in(0,1)$ for which the structure of the trajectory $\left(u^{\mu}, y^{\mu}\right)$ changes.

Remark 8.7. Consider the problem (7.1), with $g=1$, subject to the state constraint (7.2) where $h$ depends on $\mu \in[0,1]$, i.e. $y \geq h^{\mu}$ with $h^{\mu}=-1 / 8+\mu^{5} \sin (1 / \mu)$. For $\mu=0$, there is a nonessential touch point at $\tau=1 / 2$. When $\mu^{5} \sin (1 / \mu)>0$, i.e. $\mu \in \cup_{n \in \mathbb{N}^{*}}\left(\frac{1}{(2 n+1) \pi}, \frac{1}{2 n \pi}\right) \cup\left(\frac{1}{\pi}, 1\right]$, then the latter turns into a boundary arc, and when $\mu^{5} \sin (1 / \mu)<0$, i.e. $\mu \in \cup_{n \in \mathbb{N}^{*}}\left(\frac{1}{2 n \pi}, \frac{1}{(2 n-1) \pi}\right)$, the boundary arc disappear (the state constraint is not active). Therefore, for any $\varepsilon>0$ arbitrarily small, the structure changes for infinitely many values of $\mu$ in the interval $[0, \varepsilon]$. By Theorem 6.1, the computation of the directional derivatives in direction $d=1$ at point $\mu=0$ shows that problem $\left(\mathcal{P}_{d}\right)$ has zero for unique solution, and therefore the directional derivatives of the entry/exit points and jump parameters at entry times are all zero in that case.

After this general description of the homotopy path, we will focus now on the changes in the structure, i.e. when there are nonessential touch points. So consider a value $\bar{\mu} \in[0,1]$ for which $\left(u^{\bar{\mu}}, y^{\bar{\mu}}\right)$ has $N_{\text {to }} \geq 1$ (nonessential) touch points $\bar{\tau}_{i}, i=1, \ldots, N_{\mathrm{to}}$. Denote by $F_{j}$, for $j=1, \ldots, 2^{N_{\mathrm{to}}}$, the shooting mappings corresponding to all possible neighboring structures to that of $\left(u^{\bar{\mu}}, y^{\bar{\mu}}\right)$, i.e. each touch point $\bar{\tau}_{i}$ is or not converted into a boundary arc like in Section 4.2. Denote by $\bar{\theta}_{j}$ the appropriate vector of shooting parameters of $\left(u^{\bar{\mu}}, y^{\bar{\mu}}\right)$ for $F_{j}$. Thus we have

$$
F_{j}\left(\bar{\theta}_{j}, \bar{\mu}\right)=0, \quad \text { for all } j=1, \ldots, 2^{N_{\text {to }}} .
$$

For $\mu$ in the neighborhood of $\bar{\mu}$, and all $j=1, \ldots, 2^{N_{\text {to }}}$, we consider the problem:

Find $\theta$ of appropriate dimensions solution of: $\quad F_{j}(\theta, \mu)=0$. 
Lemma 8.8. Assume that $\left(\mathcal{H}_{0}\right)-\left(\mathcal{H}_{4}\right)$ hold. Let $\bar{\mu} \in[0,1]$ be such that $\left(u^{\bar{\mu}}, y^{\bar{\mu}}\right)$ has $N_{\text {to }} \geq 1$ (nonessential) touch points $\bar{\tau}_{i}, i=1, \ldots, N_{\text {to }}$. Then there exist an open neighborhood $\bar{V}_{\mu}$ of $\bar{\mu}$ and open neighborhoods $V_{j}$ of $\bar{\theta}_{j}$, $j=1, \ldots, 2^{N_{\mathrm{to}}}$, such that for all $j=1, \ldots, 2^{N_{\mathrm{to}}}$ and for all $\mu \in \bar{V}_{\mu}$, the problem (8.9) has in $V_{j}$ a unique solution $\theta_{j}^{\mu}$, and the mappings $\bar{V}_{\mu} \rightarrow V_{j}, \mu \mapsto \theta_{j}^{\mu}$, are of class $C^{1}$.

Proof. By $\left(\mathcal{H}_{3}\right)$, the touch points $\bar{\tau}_{i}$ all satisfy (1.26). By $\left(\mathcal{H}_{4}\right)$ the strong second-order sufficient condition (3.8) is satisfied, and hence the Jacobians $D_{\theta} F_{j}\left(\bar{\theta}_{j}, \bar{\mu}\right)$ are nonsingular, for all $j=1, \ldots, 2^{N_{\text {to }}}$ (by the same arguments as in the proof of Lem. 5.1). So it follows from the classical implicit function theorem that (8.9) has a locally a unique solution $\theta_{j}^{\mu}$, which is $C^{1}$ w.r.t. $\mu$.

Under the assumptions of Lemma 8.8, for $\mu \in \bar{V}_{\mu}$ and $j=1, \ldots, 2^{N_{\text {to }}}$, denote by $y_{j}^{\mu}$ the state associated with $\theta_{j}^{\mu}$, i.e. solution of (4.32)-(4.35) for the arc structure of $F_{j}$. Note that $y_{j}^{\mu}$ is well-defined on each arc of the trajectory only (not on $[0, T]$ ), since some entry times in $\theta_{j}^{\mu}$ may be greater than the corresponding exit times. Let $\hat{\theta}_{j}^{\mu}$ denote the augmented vector of shooting parameters obtained from $\theta_{j}^{\mu}$ by adding, for each touch point $\bar{\tau}_{i}$ that was not converted into a boundary arc in $F_{j}$, a zero jump parameter for the costate and an entry and exit time both equal to the unique local maximum of $g^{\mu}\left(y_{j}^{\mu}(t)\right)$ in the neighborhood of $\bar{\tau}_{i}$. Thus the augmented vectors of shooting parameters $\hat{\theta}_{j}^{\mu}$ have the same dimension for all $j$, which is also the dimension of the shooting mapping $F$ in (4.39) for which all the $N_{\text {to }}$ touch points are converted into boundary arcs. For $\mu=\bar{\mu}$, we denote the augmented vector of shooting parameters by $\bar{\theta}=\hat{\theta}_{j}^{\bar{\mu}}$, for all $j$.

Lemma 8.9. Under the assumptions of Lemma 8.8, there exists an open neighborhood $\overline{\bar{V}}_{\mu}$ of $\bar{\mu}$ such that for all $j=1, \ldots, 2^{N_{\mathrm{to}}}$, the mapping $\mu \mapsto \hat{\theta}_{j}^{\mu}$ is $C^{1}$ over $\overline{\bar{V}}_{\mu}$, and for all $\mu \in \overline{\bar{V}}_{\mu}$, the augmented vector of shooting parameters $\hat{\theta}_{j}^{\mu}$ is solution of (4.40), iff the two conditions below are satisfied:

$$
\begin{aligned}
g^{\mu}\left(y_{j}^{\mu}(t)\right) & \leq 0, & & \text { on each arc }, \\
\tau_{\mathrm{en}, j}^{\mu} & \leq \tau_{\mathrm{ex}, j}^{\mu}, & & \text { for all boundary arcs },
\end{aligned}
$$

where for each boundary arc of $F_{j}, \tau_{\mathrm{en}, j}^{\mu}$ and $\tau_{\mathrm{ex}, j}^{\mu}$ denote the components of $\theta_{j}^{\mu}$ corresponding respectively to the entry and exit point of the boundary arc.

Proof. In the neighborhood of a touch point $\bar{\tau}_{i}$ that was not converted into a boundary $\operatorname{arc}$ in $F_{j}$, for all $\mu \in \bar{V}_{\mu}$, the function $g^{\mu}\left(y_{j}^{\mu}(\cdot)\right)$ is locally well-defined and $C^{2}$. Therefore, since $\left.\frac{\mathrm{d}^{2}}{\mathrm{~d} t^{2}} g^{\bar{\mu}}\left(y^{\bar{\mu}}\right)\right|_{t=\bar{\tau}_{i}}<0$, the function that with $g^{\mu}\left(y_{j}^{\mu}\right)$ associates its (unique) local maximum time in the neighborhood of $\bar{\tau}_{i}$ is $C^{1}$, and hence, by Lemma 8.8, $\mu \mapsto \hat{\theta}_{j}^{\mu}$ is $C^{1}$. Now denote by $t_{\mathrm{en}}^{i}$ and $\nu_{i}^{1}$ respectively the entry time and jump parameter of the boundary arc associated with the touch point $\bar{\tau}_{i}$ in $\hat{\theta}_{j}^{\mu}, j=1, \ldots, 2^{N_{\text {to }}}$. By the arguments of the proof of Lemma 5.2 , we have that (8.11) is equivalent to $\nu_{i}^{1} \geq 0$ for all $i=1, \ldots, N_{\text {to }}$, and for each $i$ we have either $g^{\mu}\left(y_{j}^{\mu}\left(t_{\mathrm{en}}^{i}\right)\right)=0$ or $\nu_{i}^{1}=0$. Therefore (8.10)-(8.11) are equivalent to the condition $\Psi\left(\hat{\theta}_{j}^{\mu}, \mu\right) \in N\left(\hat{\theta}_{j}^{\mu}\right)$. The conclusion follows.

Let $j_{1}, j_{2} \in\left\{1, \ldots, 2^{N_{\text {to }}}\right\}, j_{1} \neq j_{2}$, and $\mu \in \overline{\bar{V}}_{\mu}$. Given a solution $\theta_{j_{1}}^{\mu}$ of (8.9) for $j=j_{1}$, let us explain now how to initialize the Newton algorithm in order to find a solution of (8.9) for $j=j_{2}$. The initial point $\tilde{\theta}_{j_{1}, j_{2}}^{\mu}$ is obtained from $\theta_{j_{1}}^{\mu}$ as follows:

- For every touch point $\bar{\tau}_{i}$ that was converted into a boundary arc in $F_{j_{1}}$ but not in $F_{j_{2}}$, remove from $\theta_{j_{1}}^{\mu}$ the shooting parameters associated with this boundary arc.

- For every touch point $\bar{\tau}_{i}$ that was converted into a boundary arc in $F_{j_{2}}$ but not in $F_{j_{1}}$, add to $\theta_{j_{1}}^{\mu}$ the three shooting parameters associated with this boundary $\operatorname{arc}\left(\nu^{1, i}, \tau_{\mathrm{en}}^{i}, \tau_{\mathrm{ex}}^{i}\right)$ as follows: $\nu^{1, i}=0$, and $\tau_{\mathrm{en}}^{i}$ and $\tau_{\text {ex }}^{i}$ are both equal to the unique point of local maximum of $g^{\mu}\left(y_{j_{1}}^{\mu}\right)$ in the neighborhood of $\bar{\tau}_{i}$.

Lemma 8.10. Under the assumptions of Lemma 8.8, for all $j_{1}, j_{2} \in\left\{1, \ldots, 2^{N_{\mathrm{to}}}\right\}, j_{1} \neq j_{2}$, there exists $\bar{\delta}_{j_{1}, j_{2}}>0$, such that for all $\mu,|\mu-\bar{\mu}| \leq \bar{\delta}_{j_{1}, j_{2}}$, the Newton method to solve the equation (8.9) for $j=j_{2}$ 
is convergent to $\theta_{j_{2}}^{\mu}$, whenever the initial point $\tilde{\theta}_{j_{1}, j_{2}}^{\mu}$ is obtained from the solution $\theta_{j_{1}}^{\mu}$ of $(8.9)$ for $j=j_{1}$ as explained above.

Proof. By Lemma 8.8, there exists $\rho_{j_{2}}^{\mu}>0$ such that the Newton algorithm to solve (8.9) for $j=j_{2}$ converges to $\theta_{j_{2}}^{\mu}$ for all initial point $\theta_{0}$ satisfying $\left|\theta_{0}-\theta_{j_{2}}^{\mu}\right|<\rho_{j_{2}}^{\mu}$, and this constant is uniformly positive, i.e. $\rho_{j_{2}}^{\mu} \geq \rho>0$ for all $\mu$ in a compact neighborhood of $\bar{\mu}^{1}$. Let $|\mu-\bar{\mu}| \leq \bar{\delta}_{j_{1}, j_{2}}:=\min \left(\kappa_{j_{1}}^{-1}, \kappa_{j_{2}}^{-1}\right) \rho / 3$, with $\kappa_{j}$ the Lipschitz constant of the mapping $\mu \mapsto \hat{\theta}_{j}^{\mu}$ (Lem. 8.9). Let $\bar{\theta}:=\hat{\theta}_{j_{1}}^{\bar{\mu}}=\hat{\theta}_{j_{2}}^{\bar{\mu}}$ and note that we obviously have $\tilde{\theta}_{j_{1}, j_{2}}^{\bar{\mu}}=\bar{\theta}_{j_{2}}$. It follows then that $\left|\tilde{\theta}_{j_{1}, j_{2}}^{\mu}-\theta_{j_{2}}^{\mu}\right| \leq\left|\tilde{\theta}_{j_{1}, j_{2}}^{\mu}-\tilde{\theta}_{j_{1}, j_{2}}^{\bar{\mu}}\right|+\left|\bar{\theta}_{j_{2}}-\theta_{j_{2}}^{\mu}\right| \leq\left|\hat{\theta}_{j_{1}}^{\mu}-\bar{\theta}\right|+\left|\bar{\theta}-\hat{\theta}_{j_{2}}^{\mu}\right| \leq \frac{2}{3} \rho$, from which the result follows.

We give now a theoretical proof of correctness of the algorithm.

Proposition 8.11. Assume that $\left(\mathcal{H}_{0}\right)-\left(\mathcal{H}_{2}\right),\left(\mathcal{H}_{3}^{\prime}\right)$ and $\left(\mathcal{H}_{4}\right)-\left(\mathcal{H}_{5}\right)$ hold. Then there exists $\delta_{0}>0$ such that, whenever $p_{0}$ is close enough to $\bar{p}(0)$, for all $0<\delta<\delta_{0}$ the Algorithm 8.1 follows the homotopy path previously described, and ends with a vector of shooting parameters $\theta^{1}$ of adapted dimension associated with a local solution $\left(u^{1}, y^{1}\right)$ of $\left(\mathcal{P}^{1}\right) \equiv(\mathcal{P})$. In addition, if $0<\delta<\delta_{0}$, the steps $\Delta \mu_{k}$ are not reduced by the algorithm (i.e. Newton's algorithm in Step $\mathrm{C}$ do not fail).

Proof. By $\left(\mathcal{H}_{5}\right)$, there exist finitely many values of $\mu \in(0,1), 0<\bar{\mu}_{1}<\ldots<\bar{\mu}_{m}<1$, for which the structure of the trajectory $\left(u^{\mu}, y^{\mu}\right)$ changes. By $\left(\mathcal{H}_{3}^{\prime}\right)$, this implies that for all $j=1, \ldots, m$, the trajectory associated with $\bar{\mu}_{j}$ has exactly one touch point $\bar{\tau}_{\text {to }}^{j}$. Set $\bar{\mu}_{0}:=0$ and $\bar{\mu}_{m+1}:=1$. For all $j=0, \ldots, m$, denote by $F_{j}$ the shooting mapping corresponding to the structure of $\left(u^{\mu}, y^{\mu}\right)$ for $\mu \in\left(\bar{\mu}_{j}, \bar{\mu}_{j+1}\right)$. We have $F_{j} \neq F_{j+1}$, for all $j=0, \ldots, m$.

Let $j=0, \ldots, m$. For all $\mu \in\left[\bar{\mu}_{j}, \bar{\mu}_{j+1}\right]$, by (3.8) and Lemma 8.8, there exists a constant $\rho_{j}>0$ (uniform w.r.t. $\mu$, see ${ }^{1}$ ) such that the shooting algorithm (i.e. Newton's algorithm to solve $\left.F_{j}(\theta, \mu)=0\right)$ converges to $\theta^{\mu}$ for all initial point $\theta_{0}$ satisfying $\left|\theta_{0}-\theta^{\mu}\right|<\rho_{j}$. For all $\mu, \mu^{\prime} \in\left[\bar{\mu}_{j}, \bar{\mu}_{j+1}\right]$, with $\theta^{\prime}$ the solution of the prediction step obtained from $\theta^{\mu}$ by

$$
D_{\theta} F_{j}\left(\theta^{\mu}, \mu\right)\left(\theta^{\prime}-\theta^{\mu}\right)+D_{\mu} F_{j}\left(\theta^{\mu}, \mu\right)\left(\mu^{\prime}-\mu\right)=0
$$

it is easy to see that there exists a constant $C_{j}$ such that $\left|\theta^{\prime}-\theta^{\mu^{\prime}}\right| \leq C_{j}\left|\mu-\mu^{\prime}\right|^{2}$. Therefore the convergence of the Newton algorithm to $\theta^{\mu^{\prime}}$ with the initial point $\theta^{\prime}$ is guaranteed if $\left|\mu-\mu^{\prime}\right|<\hat{\delta}:=\min _{j=0}^{m}\left(\rho_{j} / C_{j}\right)^{1 / 2}$. Now let $\delta_{0}>0$ be the minimum of $\hat{\delta}$ defined above, of all the finitely many constants $\bar{\delta}_{j_{1}, j_{2}}>0$ of Lemma 8.10 involved at the changes of structure of the trajectory, and finally of $\bar{\mu}_{j+1}-\bar{\mu}_{j}>0$, for $j=0, \ldots, m$.

Let $\delta \in\left(0, \delta_{0}\right)$. The proof of the the algorithm is by finite induction on the property below, for $k \geq 0$ :

$\left(\mathcal{A}_{k}\right)$ At each passage in the prediction step (Step B), before $k$ is increased, we have $\mu_{k}=\min (k \delta, 1), m_{k}=0$, $i_{k}=0$ and

- if $\mu_{k} \notin\left\{\bar{\mu}_{j}\right\}_{j=0, \ldots, m}, \theta_{k}=\theta^{\mu_{k}}$ is the (unique) vector of shooting parameters associated with $\left(u^{\mu_{k}}, y^{\mu_{k}}\right)$;

- if $\mu_{k}=\bar{\mu}_{j}$ for some $j=0, \ldots, m$,

* if either $k=0$ or the touch point of $\bar{\mu}_{j}$ is either inactive or a (nonessential) touch point when $\mu \rightarrow \bar{\mu}_{j}^{-}$, then $\theta_{k}$ is the vector of shooting parameters associated with $\left(u^{\mu_{k}}, y^{\mu_{k}}\right)$ that does not contain the touch point of $\bar{\mu}_{j}$ as a boundary arc of zero length;

* if the touch point of $\bar{\mu}_{j}$ is a boundary arc for $\mu \rightarrow \bar{\mu}_{j}^{-}$, then $\theta_{k}$ is the vector of shooting parameters associated with $\left(u^{\mu_{k}}, y^{\mu_{k}}\right)$ that contains the touch point of $\bar{\mu}_{j}$ as a boundary arc of zero length.

\footnotetext{
${ }^{1}$ From the proof of the Newton algorithm, it can be seen that this constant $\rho_{j}^{\mu}$ depends continuously on the Lipschitz constant of $D_{\theta} F_{j}(\cdot, \mu)$, on $\left\|D_{\theta} F_{j}\left(\theta_{j}^{\mu}, \mu\right)^{-1}\right\|^{-1}$ and on the modulus of continuity of $D_{\theta} F_{j}(\cdot, \mu)^{-1}$, and is therefore a continuous function of $\mu$.

${ }^{2}$ This constant $C_{j}$ depends on $\left\|D_{\theta} F_{j}\left(\theta^{\mu}, \mu\right)^{-1}\right\|$, on the Lipschitz constant of $D F_{j}$ and on the Lipschitz constant of the mapping $\mu \mapsto \theta^{\mu}$ on $\left[\bar{\mu}_{j}, \bar{\mu}_{j+1}\right]$ (Lem. 8.6).
} 
For $p_{0}$ sufficiently close to $\bar{p}(0)$, the initialization step of the algorithm succeeds in obtaining the initial vector of shooting parameters (reduced to the initial costate) $\theta^{0}=\theta^{\mu_{0}}$ associated with the local solution $(\bar{u}, \bar{y})$ of $\left(\mathcal{P}^{0}\right)$. So $\left(\mathcal{A}_{0}\right)$ holds. Assume now that $\left(\mathcal{A}_{k-1}\right)$ holds, and let $j \in\{0, \ldots, m+1\}$ be such that

$$
\bar{\mu}_{j}<\mu_{k-1} \leq \bar{\mu}_{j+1}
$$

We thus go through the prediction Step B and then to Step C. By $\left(\mathcal{A}_{k-1}\right)$, we try to solve, by the Newton algorithm, the equation

$$
F_{j}\left(\theta, \mu_{k}\right)=0
$$

By construction of $\delta_{0}$, the Newton algorithm succeeds and obtain a solution $\theta_{k}^{\prime}$ of (8.12). So we go to Step D. There are two cases to consider. Either (a) $\mu_{k} \leq \bar{\mu}_{j+1}$ or (b) $\mu_{k}>\bar{\mu}_{j+1}$.

In case (a), the structure of the trajectory does not change, so we obtain the vector of shooting parameters $\theta_{k}:=\theta_{k}^{\prime}=\theta^{\mu_{k}}$ associated with $\left(u^{\mu_{k}}, y^{\mu_{k}}\right)$. Therefore $m_{k} \leq 0$ and $i_{k}=0$, which shows $\left(\mathcal{A}_{k}\right)$.

In case (b), by construction of $\delta_{0}$, we have $\mu_{k} \in\left(\bar{\mu}_{j+1}, \bar{\mu}_{j+2}\right)$. Therefore $\theta^{\mu_{k}}$ is the (locally unique) solution of

$$
F_{j+1}\left(\theta, \mu_{k}\right)=0
$$

By Lemma 8.9, among all the "augmented vectors of shooting parameters" associated with one of the (two) possible neighboring structures to $\left(u^{\bar{\mu}_{j+1}}, y^{\bar{\mu}_{j+1}}\right)$, only $\theta^{\mu_{k}}$ satisfies (8.10)-(8.11). Therefore we deduce that necessarily, the augmented vector of shooting parameters $\hat{\theta}_{k}$ obtained from $\theta_{k}^{\prime}$ solution of (8.12) does not satisfy either (8.10) or (8.11), i.e. either $m_{k}>0$ or $i_{k}>0$.

Assume e.g. that $m_{k}>0$, i.e. $g^{\mu_{k}}\left(y^{\theta_{k}^{\prime}, \mu_{k}}\right)$ has positive values. Using $\left(\mathcal{H}_{2}\right)$ and Lemma 4.9 , this can only happen in the neighborhood of the touch point $\bar{\tau}_{\text {to }}^{j+1}$ of $\bar{\mu}_{j+1}$, i.e. $\tau_{k}$ is close to $\bar{\tau}_{\text {to }}^{j+1}$. Note that this is possible only if $\bar{\tau}_{\text {to }}^{j+1}$ was not converted in a boundary arc in $F_{j}$. So we go to Step A and add a boundary arc. Here, $\bar{\mu}_{j}$ having a single touch point, there are only two possible neighboring structures to that of $\left(u^{\bar{\mu}_{j}}, y^{\bar{\mu}_{j}}\right)$. Having eliminated $F_{j}$, it remains only one possible structure, i.e. with $\bar{\tau}_{\text {to }}^{j+1}$ as a boundary arc, which corresponds necessarily to $F_{j+1}$. The shooting parameters associated with this new boundary arc are initialized by (8.6), and hence we obtain an augmented vector of shooting parameters $\tilde{\theta}_{k}$, that by Lemma 8.10 belongs, by construction of $\delta_{0}$, to the neighborhood of $\theta^{\bar{\mu}_{j+1}}$ for which the Newton algorithm solving (8.13) is convergent to $\theta^{\mu_{k}}$. We thus obtain $\theta_{k}=\theta^{\mu_{k}}$, which satisfies $m_{k}=0$ and $i_{k}=0$, and therefore $\left(\mathcal{A}_{k}\right)$ holds.

The case $i_{k}>0$ is dealt with similarly, i.e. if it happens that for a boundary arc, the entry time is greater than the exit time, this can only happen in the neighborhood of the touch point $\bar{\tau}_{\text {to }}^{j+1}$ of $\bar{\mu}_{j+1}$, and this implies that this touch point is converted in a boundary arc in $F_{j}$. So we remove in Step A this boundary arc, and conclude with the same arguments that $\left(\mathcal{A}_{k}\right)$ holds again. The result follows by finite induction on $k$, since the algorithms ends for the smaller integer $k \geq \frac{1}{\delta}$.

Remark 8.12. The process of reduction of $\Delta \mu_{k}$ is not active if $\delta$ is small enough, as appears from Proposition 8.8. However, in practice we do not know what a correct value of $\Delta \mu_{k}$ is so that this reduction process is useful.

Of course when initialized with $\delta>\delta_{0}$ it may happen that Newton's method converges to a point that does not belong to the continuous path $\left(u^{\mu}, y^{\mu}\right)$, i.e., it computes another critical point, say $\left(\hat{u}^{\mu}, \hat{y}^{\mu}\right)$. If the latter satisfies conditions of Theorem 3.3, then the algorithm continues despite the jump to another branch of solutions.

Remark 8.13. We could theoretically give an explicit expression for the constant $\delta_{0}$ that ensures the convergence in Proposition 8.11, but the latter depends on constants involving, among other, bounds on the Hessian of the shooting mapping that are almost impossible to calculate. In case of ill-conditioning ( $\delta_{0}$ is very small), the convergence may be difficult, if not impossible, to achieve in practice, due to numerical errors.

Remark 8.14. Algorithm 8.1 and Proposition 8.11 can be extended to the case when $\left(\mathcal{H}_{3}\right)$ holds instead of $\left(\mathcal{H}_{3}^{\prime}\right)$. If $\left(\mathcal{H}_{3}^{\prime}\right)$ does not hold, but $\left(\mathcal{H}_{3}\right)$ do, this means that there exists $\bar{\mu} \in(0,1)$ such that $\left(u^{\bar{\mu}}, y^{\bar{\mu}}\right)$ has 
$N_{\text {to }}$ touch points, $N_{\text {to }} \geq 2$. If the structure of the shooting mapping changes at this point, there are a priori $2^{N_{\text {to }}}$ possibilities for the new structure when $\mu \rightarrow \hat{\mu}^{+}$. It is possible to enumerate all of them, i.e. solve (8.9), for all $j=1, \ldots, 2^{N_{\text {to }}}$, for $\mu>\bar{\mu}$ close to $\bar{\mu}$. Lemma 8.9 ensures that if (8.10)-(8.11) are satisfied for some $j$, then we have found the new structure, and Lemma 5.1 ensures that (8.10)-(8.11) will be satisfied for at least one $j$.

A possibility that may reduces the enumeration is to use the directional differentiability of solutions in Theorem 6.1. One can e.g. solve the problem $\left(\mathcal{P}_{d}\right)$, and whenever the variation $\sigma_{d, i}^{\mathrm{ex}}-\sigma_{d, i}^{\mathrm{en}}$ given by $(6.8)$ is positive (resp. negative), this tells us that the touch point $\tau_{\text {to }}^{i}$ have to be converted into boundary arc (resp. removed from the shooting mapping). For touch points such that $\sigma_{d, i}^{\mathrm{ex}}-\sigma_{d, i}^{\mathrm{en}}=0$, this gives no information on $\tau_{\text {to }}^{i}$ so it possibly remains different possibilities to enumerate.

\subsection{Numerical implementation}

The convergence of the algorithm presented in the previous subsections is illustrated on the academic problem below:

$$
\begin{aligned}
& (\mathcal{P}) \quad \min \int_{0}^{1}\left(\frac{u^{2}(t)}{2}+g(t) y(t)\right) \mathrm{d} t \\
& \text { s.t. } \quad \dot{y}(t)=u(t), \quad y(0)=y(1)=0, \quad y(t) \geq h
\end{aligned}
$$

with

$$
g(t):=g_{0}(c-\sin (\alpha t)), \quad c, \alpha>0 .
$$

The time is introduced as a state variable, and let $\mu=\left(h-h_{0}\right) /\left(h_{1}-h_{0}\right)$ be the homotopy parameter, with $h_{0}=\min \bar{y}(t)$, for $\bar{y}$ the solution of the problem without the state constraint, and $h_{1}=h$ the desired value of the state constraint. Numerical values of constants are taken equal to

$$
g_{0}:=10, \quad \alpha=10 \pi, \quad c=0.1, \quad h_{1}=-0.001 .
$$

The algorithm is initialized with the value $p_{0}=0$, and $\delta=1 / 5$ to initialize the steps $\Delta \mu_{k}$. Let us comment Figure 3 where the results of the algorithm are presented. The algorithm reduces the step $\Delta \mu_{k}$ once, in the next to last iteration, since the Newton algorithm was not converging, meaning here that it was not converging quadratically. Thus the solution was computed for the values $\mu_{0}=0, \mu_{k}=k \delta=k / 5$ for $k=1, \ldots, 4, \mu_{5}=9 / 10$ and $\mu_{6}=1$. We plotted in dark blue the state $y_{k}$ solution of $\left(\mathcal{P}^{\mu_{k}}\right)$ obtained at the exit of the WHILE loop when $m_{k}=i_{k}=0$, for $k=0, \ldots, 6$. In light blue we plotted the previous iterations, including the states obtained when $m_{k}>0$ at the exit of the WHILE loop (so we can see the algorithm add a boundary arc at the following iteration when this happens).

For $k=0$, we just have the solution of the unconstrained problem. For $k=1$, the algorithm adds a single boundary arc around time $t=0.55$. At each iteration $k=2,3,4$, the algorithm detects that the state constraint is violated so it adds a boundary arc. So for $k=4$ we have $\mu_{k}=0.8$ and four boundary arcs. Then the algorithm tries to pursue the homotopy with $\mu=1$. It detects that it has to add a boundary arc but Newton algorithm fails. Therefore it decreases the step and obtained the solution for $\mu_{5}$ (see the figure for $k=5$ ) that has a fifth boundary arc. It then increases $\mu$ to $\mu_{6}=1$ and obtain the solution of $(\mathcal{P})$ which exhibits five boundary arcs.

At each passage in the Newton algorithm (Step C), the latter converges very rapidly in 2 or 3 iterations (for the tolerance $\left.\left|F\left(\theta_{k}, \mu_{k}\right)\right|_{\infty} \leq 10^{-10}\right)$ excepted of course the time it failed because $\Delta \mu_{k}$ was too large, and at the very last passage (which requires 5 iterations).

Finally, let us check that the uniform strict complementarity hypothesis $\left(\mathcal{H}_{2}\right)$ is satisfied. On a boundary arc, (4.3) gives

$$
u_{b}+p_{1}-\eta_{1}=0 \quad \text { with } u_{b}=0,
$$

i.e. $p_{1}=\eta_{1}$. Hence, $\dot{\eta}_{1} \leq \beta<0$ on boundary arcs iff $p_{1}$ is (uniformly) decreasing. This is the case, see the figure bottom right in Figure 3 on which we plotted $p_{1}$ for the final solution for $\mu_{6}=1$ (the portions corresponding to boundary arcs are plotted in red). We can also check similarly that this uniform strict complementarity assumption is satisfied as well for all other values of $\mu_{k}, k=1, \ldots, 5$. 
$k=0$
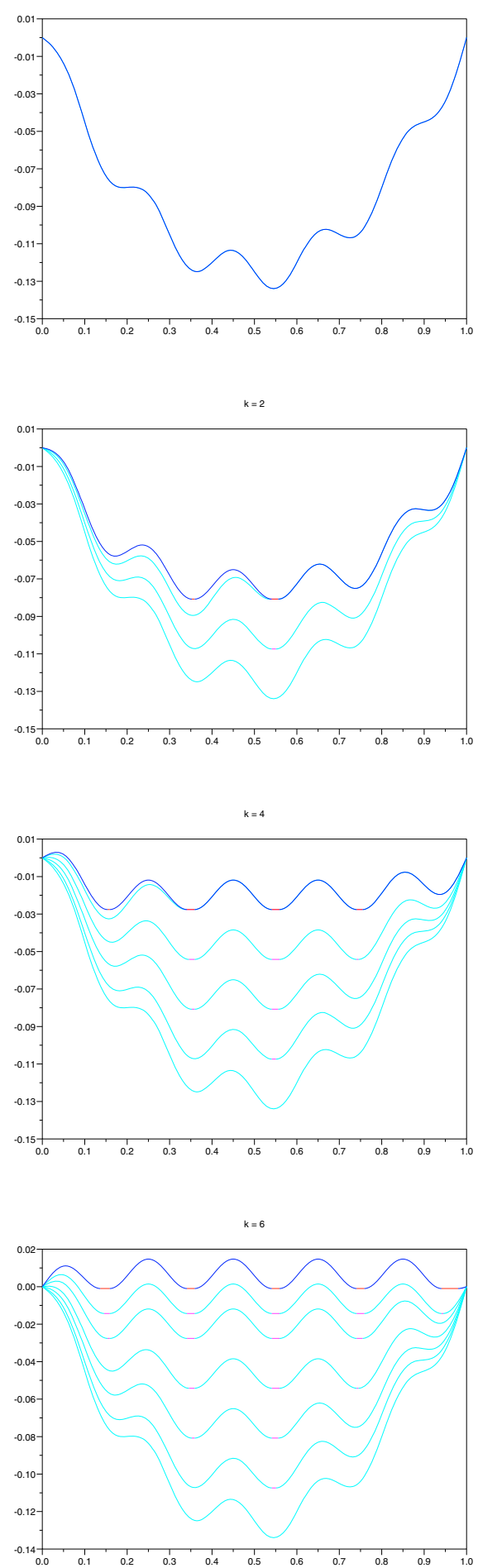

$k=1$

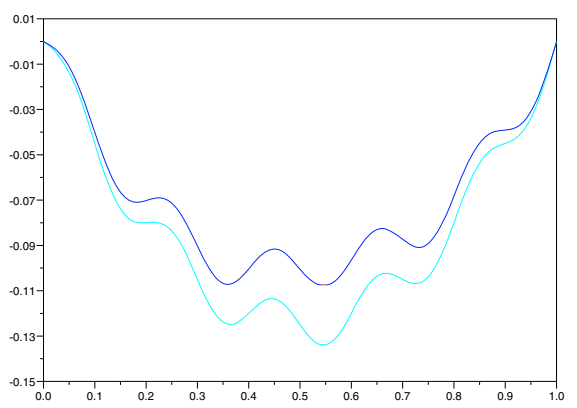

$k=3$
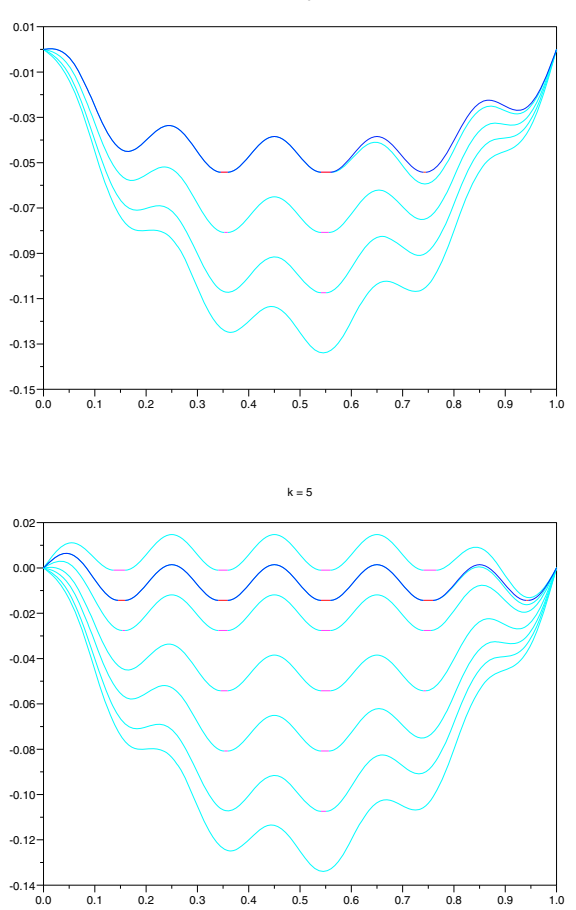

costate $(k=\theta)$

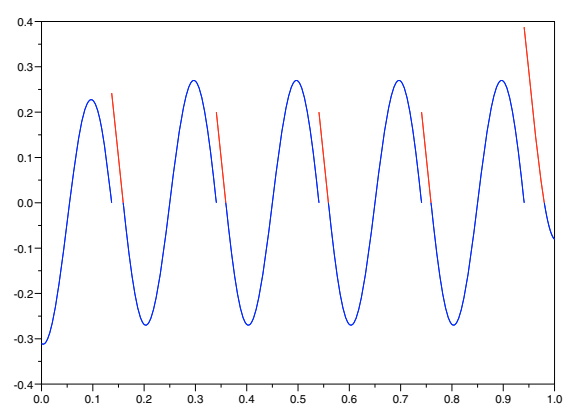

FigURE 3. Iterations of the homotopy algorithm and costate $p_{1}($ for $k=6)$. 


\section{Proof of Theorem 2.1}

We start by the proof of Lemma 2.3, then give that of Lemma 2.5, and finally that of Theorem 2.1.

Proof of Lemma 2.3. Let $\delta>0$. By continuity of the mapping $(u, \mu) \mapsto g^{\mu}\left(y_{u}^{\mu}\right)$, there exists $\delta>0$, such that for $n$ large enough (this is precisely assertion $(\mathrm{S} 1)$ ),

$$
I\left(g^{\mu_{n}}\left(y_{n}\right)\right) \subset \Omega^{\delta}:=\cup_{i=1}^{N} \Omega_{i}^{\delta} .
$$

The first assertion of the lemma is a classical consequence of Robinson's constraint qualification (1.17) (see e.g. [8], Prop. 4.43). By Lemma 1.2, reducing $\delta$ if necessary, the mapping (1.23) is onto. $\operatorname{Since} \operatorname{supp}\left(\mathrm{d} \eta_{n}\right) \subset$ $I\left(g^{\mu_{n}}\left(y_{n}\right)\right) \subset \Omega^{\delta}$ by (9.1), the second assertion follows from [8], Proposition 4.44 and Remark 4.45(i), meaning that

$$
\sup _{\Phi \in W^{1, \infty}(0, T), \Phi \neq 0} \frac{\left|\int_{0}^{T} \Phi(t)\left(\mathrm{d} \eta_{n}-\mathrm{d} \eta\right)(t) \mathrm{d} t\right|}{\|\Phi\|_{1, \infty}} \underset{n \rightarrow+\infty}{\longrightarrow} 0 .
$$

Let $p_{n}^{1}$ and $\eta_{n}^{1}$ be the multipliers associated with the stationary point $\left(u_{n}, y_{n}\right)$ of $\left(\mathcal{P}^{\mu_{n}}\right)$ by $(2.5)-(2.6)$. By (2.7),

$$
\frac{\mathrm{d}}{\mathrm{d} t}\left(p_{n}^{1}-\bar{p}^{1}\right)=\left(p_{n}^{1}-\bar{p}^{1}\right) f_{y}(\bar{u}, \bar{y})+g_{y}^{(1)}(\bar{u}, \bar{y})\left(\eta_{n}^{1}-\bar{\eta}^{1}\right)+r_{n}(t) \quad \text { a.e. on }[0, T],
$$

with $\left\|r_{n}\right\|_{\infty} \rightarrow 0$ when $n \rightarrow+\infty$. By Gronwall's lemma, there exists a constant $C>0$ such that

$$
\left|p_{n}^{1}(t)-\bar{p}^{1}(t)\right| \leq C\left|\phi_{y}^{\mu_{n}}\left(y_{n}(T)\right)-\phi_{y}^{\mu_{0}}(\bar{y}(T))\right|+C \int_{t}^{T}\left|\eta_{n}^{1}(s)-\bar{\eta}^{1}(s)\right| \mathrm{d} s+o_{\infty}(1) \leq C\left\|\eta_{n}^{1}-\bar{\eta}^{1}\right\|_{1}+o_{\infty}(1),
$$

where $o_{\infty}(1)$ denotes a function that goes to zero in $L^{\infty}$ when $n \rightarrow+\infty$. Let us show that $\eta_{n}^{1} \rightarrow \bar{\eta}^{1}$ in $L^{1}$. The sequence $\left(\mathrm{d} \eta_{n}\right)_{n \in \mathbb{N}^{*}}$ being bounded in $\mathcal{M}[0, T]$ by the first assertion (1), it follows that $\left(\eta_{n}^{1}\right)_{n \in \mathbb{N}^{*}}$ is bounded in $B V$, for the norm $\|\eta\|_{B V}=\|\eta\|_{1}+\|\mathrm{d} \eta\|_{\mathcal{M}}$. By the compactness theorem in $B V$ [2], Theorem 3.23, there exists a subsequence $\left(\eta_{\psi(n)}^{1}\right)_{n \in \mathbb{N}^{*}}$ converging in $L^{1}$ to some $\tilde{\eta} \in B V(0, T)$, and such that $\mathrm{d} \eta_{\psi(n)} \stackrel{*}{\rightarrow}-\mathrm{d} \tilde{\eta}$ in $\mathcal{M}[0, T]$. It suffices then to show that necessarily, $-\mathrm{d} \tilde{\eta}=\mathrm{d} \bar{\eta}$ and $\tilde{\eta}=\bar{\eta}^{1}$ in order to obtain the convergence of the whole sequence $\left(\eta_{n}^{1}\right)_{n \in \mathbb{N}^{*}}$ to $\bar{\eta}^{1}$ in $L^{1}$. So let us do that. The space $W^{1, \infty}(0, T)$ being dense in $C^{0}[0, T]$, it follows easily from (9.2) that $\mathrm{d} \eta_{n} \stackrel{*}{\rightarrow} \mathrm{d} \bar{\eta}$, and hence $-\mathrm{d} \tilde{\eta}=\mathrm{d} \bar{\eta}$. Thus $\tilde{\eta}$ equals $\bar{\eta}$ up to a constant. Using Fubini's theorem and (9.2), we obtain

$$
\int_{0}^{T} \eta_{n}^{1}(t) \mathrm{d} t=\int_{0}^{T} s \mathrm{~d} \eta_{n}(s) \underset{n \rightarrow+\infty}{\longrightarrow} \int_{0}^{T} s \mathrm{~d} \bar{\eta}(s)=\int_{0}^{T} \bar{\eta}^{1}(t) \mathrm{d} t
$$

implying finally that $\tilde{\eta}=\bar{\eta}$, and consequently, that $\eta_{n}^{1} \rightarrow \bar{\eta}^{1}$ in $L^{1}$. By (9.3), we deduce then that $p_{n}^{1} \rightarrow \bar{p}^{1}$ uniformly over $[0, T]$.

Finally, for $\left\|u_{n}-\bar{u}\right\|_{\infty}$ small enough, $\left|\left(g^{\mu_{n}}\right)_{u}^{(1)}\left(u_{n}, y_{n}\right)\right| \geq \beta / 2>0$ on $\Omega^{\delta}$, so by $(2.8)$ we have on $\Omega^{\delta}$ :

$$
\eta_{n}^{1}=-\frac{H_{u}^{\mu_{n}}\left(u_{n}, y_{n}, p_{n}^{1}\right)}{\left(g^{\mu_{n}}\right)_{u}^{(1)}\left(u_{n}, y_{n}\right)} \rightarrow-\frac{H_{u}^{\mu_{0}}\left(\bar{u}, \bar{y}, \bar{p}^{1}\right)}{\left(g^{\mu_{0}}\right)_{u}^{(1)}(\bar{u}, \bar{y})}=\bar{\eta}^{1} \quad \text { uniformly on } \Omega^{\delta}
$$

and $\eta_{n}^{1}$ is piecewise constant on $[0, T] \backslash \Omega^{\delta}$, which shows the last assertion.

Proof of Lemma 2.5. Let $(u, y)$ be a stationary point of $\left(\mathcal{P}^{\mu}\right)$ with multipliers $\left(p^{1}, \eta^{1}\right)$ given by $(2.5)-(2.6)$. By time derivation of (2.8), we have, using the augmented Hamiltonian (1.8),

$$
\tilde{H}_{u u}^{\mu}\left(u, y, p^{1}, \eta^{1}\right) \dot{u}+\tilde{H}_{u y}^{\mu}\left(u, y, p^{1}, \eta^{1}\right) f^{\mu}(u, y)-\tilde{H}_{y}^{\mu}\left(u, y, p^{1}, \eta^{1}\right) f_{u}^{\mu}(u, y)+\left(g^{\mu}\right)_{u}^{(1)}(u, y) \dot{\eta}^{1}=0 .
$$


For $\left\|\mu-\mu_{0}\right\|$ and $\|u-\bar{u}\|_{\infty}$ small enough, then $\|y-\bar{y}\|_{\infty}$ is arbitrarily small, as well as $\left\|p^{1}-\bar{p}^{1}\right\|_{\infty}$ and $\left\|\eta^{1}-\bar{\eta}^{1}\right\|_{\infty}$ by Lemma 2.3. Consequently, for $(u, \mu)$ close enough to $\left(\bar{u}, \mu_{0}\right)$, we have by $(1.20)$ that $\tilde{H}_{u u}^{\mu}\left(u, y, p^{1}, \eta^{1}\right) \geq \alpha / 2$ on $[0, T]$. Multiplying $(9.4)$ by $\left(g^{\mu}\right)_{u}^{(1)}(u, y) / \tilde{H}_{u u}^{\mu}\left(u, y, p^{1}, \eta^{1}\right)$, we obtain that

$$
\left(g^{\mu}\right)_{u}^{(1)}(u, y) \dot{u}+\frac{\left(g^{\mu}\right)_{u}^{(1)}(u, y)^{2}}{\tilde{H}_{u u}^{\mu}\left(u, y, p^{1}, \eta^{1}\right)} \dot{\eta}^{1} \rightarrow g_{u}^{(1)}(\bar{u}, \bar{y}) \dot{\bar{u}}+\frac{g_{u}^{(1)}(\bar{u}, \bar{y})^{2}}{\tilde{H}_{u u}\left(\bar{u}, \bar{y}, \bar{p}^{1}, \bar{\eta}^{1}\right)} \dot{\bar{\eta}}^{1}
$$

uniformly over $[0, T]$. In view of $(1.5)-(1.6)$, it follows that

$$
\left(g^{\mu}\right)^{(2)}(\dot{u}, u, y)+\frac{\left(g^{\mu}\right)_{u}^{(1)}(u, y)^{2}}{\tilde{H}_{u u}^{\mu}\left(u, y, p^{1}, \eta^{1}\right)} \dot{\eta}^{1} \rightarrow g^{(2)}(\dot{\bar{u}}, \bar{u}, \bar{y})+\frac{g_{u}^{(1)}\left(\bar{u}, \bar{y}^{2}\right.}{\tilde{H}_{u u}\left(\bar{u}, \bar{y}, \bar{p}^{1}, \bar{\eta}^{1}\right)} \dot{\bar{\eta}}^{1}
$$

again, uniformly over $[0, T]$.

Now on every $\Omega_{i}^{\delta}$, for small enough $\delta>0$, we have by (A5)-(A6), (1.27) and (1.21) the existence of a constant $\kappa_{1}>0$ such that either $g^{(2)}(\dot{\bar{u}}, \bar{u}, \bar{y})<-\kappa_{1}$ and $\dot{\bar{\eta}}^{1}=0$, or $g^{(2)}(\dot{\bar{u}}, \bar{u}, \bar{y})=0,\left|g_{u}^{(1)}(\bar{u}, \bar{y})\right| \geq \kappa_{1}$ and $\dot{\bar{\eta}}^{1} \leq-\kappa_{1}$. It follows that, for some $\kappa_{2}>0, \delta$ small enough and $(\mu, u)$ close to $\left(\mu_{0}, \bar{u}\right)$,

$$
\left(g^{\mu}\right)^{(2)}(\dot{u}, u, y)+\frac{\left(g^{\mu}\right)_{u}^{(1)}(u, y)^{2}}{\tilde{H}_{u u}^{\mu}\left(u, y, p^{1}, \eta^{1}\right)} \dot{\eta}^{1} \leq-\kappa_{2} \quad \text { on } \Omega_{i}^{\delta} .
$$

If $g^{\mu}(y(t))<0$, then $\dot{\eta}^{1}(t)=0$, and hence, $\left(g^{\mu}\right)^{(2)}(\dot{u}, u, y)(t)<-\kappa_{2} / 2$. But on an interior arc included in $\Omega_{i}^{\delta}, g^{\mu}(y)$ would attain its minimum at some point $t$ where $\left(g^{\mu}\right)^{(2)}(\dot{u}, u, y)(t) \geq 0$, which gives the desired contradiction.

Remark 9.1. It follows from (9.7) that the property of uniform strict complementarity is stable, in the sense that if the state constraint is active, then $\dot{\eta}^{1}$ remains uniformly far from zero (uniformly over $[0, T]$ ).

Now we are ready to give the proof of Theorem 2.1.

Proof of Theorem 2.1. Assertion (S1) is immediate, and (S3) follows directly from Lemma 2.5 since there is no interior arc of $(u, y)$ in $\Omega_{i}^{\delta}$. In view of Lemma 2.5, to complete the proof of (S2), it remains to show that $\Omega_{i}^{\delta} \cap I\left(g^{\mu}(y)\right)$ is an interval of positive measure, i.e. a boundary arc. Assume that this is false. Then there exist a stable extension $\left(\mathcal{P}^{\mu}\right)$, sequences $u_{n} \rightarrow \bar{u}$ in $L^{\infty}, \mu_{n} \rightarrow \mu_{0}$, and $\left(u_{n}, y_{n}\right)$ a stationary point of $\left(\mathcal{P}^{\mu_{n}}\right)$, such that for all $n, \Omega_{i}^{\delta} \cap I\left(g^{\mu_{n}}\left(y_{n}\right)\right)$ is either empty or a singleton by Lemma 2.5. Taking if necessary a subsequence, this implies that there exists an interval of positive measure $\left(t_{1}, t_{2}\right) \subset\left[\bar{t}_{i}^{\mathrm{en}}, \bar{t}_{i}^{\mathrm{ex}}\right]$, such that $\left(t_{1}, t_{2}\right) \cap I\left(g^{\mu_{n}}\left(y_{n}\right)\right)=\emptyset$ for all $n$, and hence, $\left(t_{1}, t_{2}\right) \cap \operatorname{supp}\left(\mathrm{d} \eta_{n}\right)=\emptyset$. Let $\varphi$ be a $C^{\infty}$ function with support in $\left[t_{1}, t_{2}\right]$ which is positive on $\left(t_{1}, t_{2}\right)$. Then we have $\int_{0}^{T} \varphi(t) \mathrm{d} \eta_{n}(t)=0$, for all $n$. But by (A5), $\bar{\eta}$ has a positive density over $\left(t_{1}, t_{2}\right)$, and hence, $\int_{0}^{T} \varphi(t) \mathrm{d} \bar{\eta}(t)>0$, which contradicts the second assertion in Lemma 2.3. This achieves the proof of assertion (S2).

Acknowledgements. The authors thank an anonymous referee for his remarks that helped to improve the paper.

\section{REFERENCES}

[1] E.L. Allgower and K. Georg, Numerical continuation methods, Springer Series in Computational Mathematics 13. SpringerVerlag, Berlin (1990).

[2] L. Ambrosio, N. Fusco and D. Pallara, Functions of bounded variation and free discontinuity problems, Oxford Mathematical Monographs. The Clarendon Press Oxford University Press, New York (2000).

[3] P. Berkmann and H.J. Pesch, Abort landing in windshear: optimal control problem with third-order state constraint and varied switching structure. J. Optim. Theory Appl. 85 (1995) 21-57. 
[4] J.F. Bonnans and A. Hermant, Conditions d'optimalité du second ordre nécessaires ou suffisantes pour les problèmes de commande optimale avec une contrainte sur l'état et une commande scalaires. C. R. Math. Acad. Sci. Paris $\mathbf{3 4 3}$ (2006) $473-478$.

[5] J.F. Bonnans and A. Hermant, Second-order analysis for optimal control problems with pure state constraints and mixed control-state constraints. Ann. Inst. H. Poincaré Anal. Non Linéaire (to appear).

[6] J.F. Bonnans and A. Hermant, Well-posedness of the shooting algorithm for state constrained optimal control problems with a single constraint and control. SIAM J. Control Optim. 46 (2007) 1398-1430.

[7] J.F. Bonnans and A. Hermant, No gap second order optimality conditions for optimal control problems with a single state constraint and control. Math. Programming, Ser. B (2007) DOI: 10.1007/s10107-007-0167-8.

[8] J.F. Bonnans and A. Shapiro, Perturbation analysis of optimization problems. Springer-Verlag, New York (2000).

[9] A.E. Bryson, W.F. Denham and S.E. Dreyfus, Optimal programming problems with inequality constraints I: Necessary conditions for extremal solutions. AIAA Journal 1 (1963) 2544-2550.

[10] R. Bulirsch, F. Montrone and H.J. Pesch, Abort landing in the presence of windshear as a minimax optimal control problem. II. Multiple shooting and homotopy. J. Optim. Theory Appl. 70 (1991) 223-254.

[11] P. Deuflhard, Newton methods for nonlinear problems, Affine invariance and adaptive algorithms, Springer Series in Computational Mathematics 35. Springer-Verlag, Berlin (2004).

[12] A.L. Dontchev and W.W. Hager, Lipschitzian stability for state constrained nonlinear optimal control. SIAM J. Control Optim. 36 (1998) 698-718 (electronic).

[13] N. Dunford and J. Schwartz, Linear operators, Vols. I and II. Interscience, New York (1958), (1963).

[14] J. Gergaud and T. Haberkorn, Homotopy method for minimum consumption orbit transfer problem. ESAIM: COCV 12 (2006) 294-310 (electronic).

[15] W.W. Hager, Lipschitz continuity for constrained processes. SIAM J. Control Optim. 17 (1979) 321-338.

[16] A. Haraux, How to differentiate the projection on a convex set in Hilbert space. Some applications to variational inequalities. J. Math. Soc. Japan 29 (1977) 615-631.

[17] R.F. Hartl, S.P. Sethi and R.G. Vickson, A survey of the maximum principles for optimal control problems with state constraints. SIAM Review 37 (1995) 181-218.

[18] A.D. Ioffe and V.M. Tihomirov, Theory of Extremal Problems. North-Holland Publishing Company, Amsterdam (1979). Russian Edition: Nauka, Moscow (1974).

[19] D.H. Jacobson, M.M. Lele and J.L. Speyer, New necessary conditions of optimality for control problems with state-variable inequality contraints. J. Math. Anal. Appl. 35 (1971) 255-284.

[20] K. Malanowski, Two-norm approach in stability and sensitivity analysis of optimization and optimal control problems. Adv. Math. Sci. Appl. 2 (1993) 397-443.

[21] K. Malanowski, Stability and sensitivity of solutions to nonlinear optimal control problems. J. Appl. Math. Optim. 32 (1995) 111-141.

[22] K. Malanowski, Sufficient optimality conditions for optimal control subject to state constraints. SIAM J. Control Optim. 35 (1997) 205-227.

[23] K. Malanowski and H. Maurer, Sensitivity analysis for state constrained optimal control problems. Discrete Contin. Dynam. Systems 4 (1998) 241-272.

[24] P. Martinon and J. Gergaud, An application of PL continuation methods to singular arcs problems, in Recent advances in optimization, Lect. Notes Econom. Math. Systems 563, Springer, Berlin (2006) 163-186.

[25] H. Maurer, On the minimum principle for optimal control problems with state constraints. Schriftenreihe des Rechenzentrum 41, Universität Münster (1979).

[26] H. Maurer and H.J. Pesch, Solution differentiability for nonlinear parametric control problems. SIAM J. Control Optim. 32 (1994) 1542-1554.

[27] F. Mignot, Contrôle dans les inéquations variationnelles elliptiques. J. Funct. Anal. 22 (1976) $130-185$.

[28] L.S. Pontryagin, V.G. Boltyanskii, R.V. Gamkrelidze and E.F. Mishchenko, The mathematical theory of optimal processes. Translated from the Russian by K.N. Trirogoff; L.W. Neustadt Ed., Interscience Publishers John Wiley \& Sons, Inc. New YorkLondon (1962).

[29] S.M. Robinson, First order conditions for general nonlinear optimization. SIAM J. Appl. Math. 30 (1976) 597-607.

[30] S.M. Robinson, Stability theorems for systems of inequalities, part II: Differentiable nonlinear systems. SIAM J. Numer. Anal. 13 (1976) 497-513.

[31] S.M. Robinson, Strongly regular generalized equations. Math. Oper. Res. 5 (1980) 43-62.

[32] J. Sokolowski, Sensitivity analysis of control constrained optimal control problems for distributed parameter systems. SIAM J. Control Optim. 25 (1987) 1542-1556.

[33] J. Stoer and R. Bulirsch, Introduction to Numerical Analysis. Springer-Verlag, New York (1993). 